\title{
Reflection Positive Stochastic Processes Indexed by Lie Groups
}

\author{
Palle E.T. JORGENSEN ${ }^{\dagger}$, Karl-Hermann NEEB ${ }^{\ddagger}$ and Gestur ÓLAFSSON $§$ \\ $\dagger$ Department of Mathematics, The University of Iowa, Iowa City, IA 52242, USA \\ E-mail: palle-jorgensen@uiowa.edu \\ ‡ Department Mathematik, FAU Erlangen-Nürnberg, \\ Cauerstrasse 11, 91058-Erlangen, Germany \\ E-mail: neeb@math.fau.de \\ $\S$ Department of mathematics, Louisiana State University, Baton Rouge, LA 70803, USA \\ E-mail: olafsson@math.lsu.edu
}

Received October 28, 2015, in final form June 09, 2016; Published online June 21, 2016

http://dx.doi.org/10.3842/SIGMA.2016.058

\begin{abstract}
Reflection positivity originates from one of the Osterwalder-Schrader axioms for constructive quantum field theory. It serves as a bridge between euclidean and relativistic quantum field theory. In mathematics, more specifically, in representation theory, it is related to the Cartan duality of symmetric Lie groups (Lie groups with an involution) and results in a transformation of a unitary representation of a symmetric Lie group to a unitary representation of its Cartan dual. In this article we continue our investigation of representation theoretic aspects of reflection positivity by discussing reflection positive Markov processes indexed by Lie groups, measures on path spaces, and invariant gaussian measures in spaces of distribution vectors. This provides new constructions of reflection positive unitary representations.
\end{abstract}

Key words: reflection positivity; stochastic process; unitary representations

2010 Mathematics Subject Classification: 22E45; 60G15; 81S40

\section{Contents}

1 Introduction 2

2 Reflection positive representations $\quad 4$

$\begin{array}{lll}3 & \text { Reflection positive Lie group actions on measure spaces } & \mathbf{7}\end{array}$

$3.1(G, S, \tau)$-measure spaces $\ldots \ldots \ldots \ldots \ldots \ldots \ldots$

3.2 Stochastic processes indexed by Lie groups . . . . . . . . . . . . . . . . . 12

3.3 Associated positive semigroup structures and reconstruction . . . . . . . . . . . 15

3.4 Klein's characterization of the Markov case . . . . . . . . . . . . . . . . . 22

3.5 Total subsemigroups of Lie groups . . . . . . . . . . . . . . . . . . . . 23

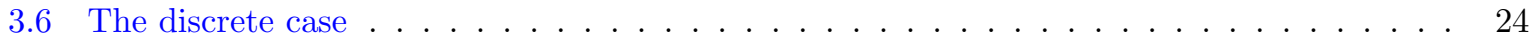

4 Measures on path spaces for topological groups $\quad \mathbf{2 5}$

4.1 One-parameter convolution semigroups of measures on polish groups . . . . . . . . . . 25

4.2 Standard path space structures for locally compact groups . . . . . . . . . . . . . . . . 29

5 Gaussian $(G, S, \tau)$-probability spaces $\quad 31$

5.1 Second quantization and gaussian processes $\ldots \ldots \ldots \ldots \ldots \ldots \ldots \ldots$

5.2 Application to reflection positive representations . . . . . . . . . . . . . . 34

5.3 Equivalence of gaussians measures for reproducing kernel Hilbert spaces . . . . . . . . . . 34 
5.4 Gaussian measures on distributions on Lie groups . . . . . . . . . . . . . . 36

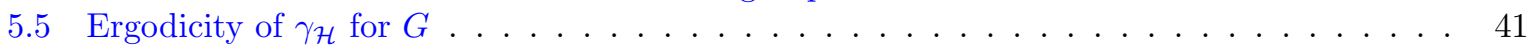

A Continuity of a stochastic process on $G$

B Markov kernels

References

\section{Introduction}

Reflection positivity is one of the cornerstones in constructive quantum field theory. It was first formulated in the fundamental work of Osterwalder and Schrader [57, 58], see also [20]. Mathematically, a quantum physical system corresponds to a unitary representation of the corresponding symmetry group $G$. In euclidean quantum field theory this group is the euclidean motion group and in relativistic quantum field theory the symmetry group is the Poincaré group. Reflection positivity enters the picture when it comes to passing from euclidean quantum fields to relativistic ones. The time reflection and the passage to imaginary time, sometimes called Wick rotation, changes the standard euclidean inner product into the Lorentz form of relativity. The time reflection corresponds to an involution $\tau$ of the euclidean motion group $G$ and then the duality of symmetric Lie algebras $\mathfrak{g}=\mathfrak{g}^{\tau} \oplus \mathfrak{g}^{-\tau} \leftrightarrow \mathfrak{g}^{c}=\mathfrak{g}^{\tau} \oplus i \mathfrak{g}^{-\tau}$ leads to a duality between the Lie algebra of the euclidean motion group and that of the Poincaré group.

In the following we call a pair $(G, \tau)$, consisting of a Lie group $G$ and an involutive automorphism $\tau: G \rightarrow G$ a symmetric Lie group. On the group level, the aforementioned duality is implemented by the Cartan duality (or $c$-duality for short) $G \leftrightarrow G^{c}$, where $G^{c}$ is the simply connected Lie group with Lie algebra $\mathfrak{g}^{c}$. Passing from real to imaginary time and from the euclidean motion group to the universal covering of the (identity component of the) Poincaré group is an important special case of $c$-duality which has been studied independently from the quantum field theoretic context.

In addition to $c$-duality, the other three basic notions considered in the basic theory of reflection positivity are that of reflection positive Hilbert spaces, reflection positive kernels, and reflection positive representations. A reflection positive representation together with the Osterwalder-Schrader quantization leads to an infinitesimally unitary representation of the Lie algebra $\mathfrak{g}^{c}$. The problem in representation theory is then to determine if this representation arises as the derived representation of a unitary representation of $G^{c}$, which establishes a passage from a unitary representations of $G$ to one of $G^{c}$.

This integration process is often accomplished using reflection positive kernels and geometric actions of the Lie group or its Lie algebra [46] or, as in the case of the Lüscher-Mack theorem [45], using semigroups and invariant cones. A second step is then to determine the resulting representation in terms of decomposition into irreducible representation. For further representation theoretic results related to reflection positivity, we refer to [26, 33, 34, 35, 36, 49, 50, 51, 56, 60]. Our present paper concentrates on various mathematical aspects of constructions of reflection positive representations: infinite-dimensional analysis, functional integration and gaussian measures, and stochastic processes. On the way we recall several basic facts in these areas to make this article more self-contained.

Shortly after the groundbreaking work of Osterwalder and Schrader, A. Klein and L. Landau built a bridge between reflection positivity, path spaces and stochastic processes [40, 41, 42]. One of our goals in this article is to connect the ideas of Klein and Landau to representation theory and our previous work on reflection positive representations [35, 36, 49, 50, 51]. This is done by replacing the real line, viewed as continuous time, by an arbitrary Lie group $G$, not necessarily finite-dimensional, and the positive time axes $\mathbb{R}_{+}$by a semigroup $S \subset G$ invariant 
under $s \mapsto s^{\#}=\tau(s)^{-1}$. This leads naturally to the concepts of $(G, \tau)$-measure spaces, reflection positive measure spaces, and a positive semigroup structures introduced in Section 3 of this article. Many concepts extend naturally to general triples $(G, S, \tau)$. But in the generalization of the Abel-Klein reconstruction theorem (Theorem 3.22) which reconstructs a $(G, S, \tau)$-measure space from a positive semigroup structure, we need to assume that $G=S \cup S^{-1}$; then $S$ is called total. It would be interesting to see how far our techniques can be extended beyond total subsemigroups.

In the following section we turn back to the classical case where the symmetric Lie group is $\left(\mathbb{R}, \mathbb{R}_{+},-\mathrm{id}\right)$, but the measure space is the path space $P(Q)=Q^{\mathbb{R}}$ of a polish topological group $Q$, mostly assumed locally compact. If $\nu$ is a measure on $Q$ and $\left(\mu_{t}\right)_{t \geq 0}$ is a convolution semigroup of symmetric probability measures on $Q$ satisfying $\nu * \mu_{t}=\nu$ for every $t>0$, then the reconstruction theorem applies to the corresponding $\mathbb{R}_{+}$-action on $L^{2}(Q, \nu)$ and leads to an invariant probability measure on the path space $Q^{\mathbb{R}}$ corresponding to a reflection positive representation (Theorem 4.9). However, the corresponding measure on $P(Q)$ is the product of the measure $\nu$, on the constant paths, and a probability measure on the pinned paths

$$
P_{*}(Q):=\{\omega \in P(Q): \omega(0)=\mathbf{1}\},
$$

which is also invariant under a suitable one-parameter group of transformations. Since we presently do not know how to obtain a similar factorization if $\left(\mathbb{R}, \mathbb{R}_{+},-\mathrm{id}_{\mathbb{R}}\right)$ is replaced by some $(G, S, \tau)$, we discuss the one-dimensional case in some detail.

One of the advantages of the reflection positivity condition is that it allows to construct representations of a symmetric Lie group (classically the euclidean motion group) in $L^{2}$-spaces of measures on spaces of distributions, which after the Osterwalder-Schrader quantization lead to unitary representations of the $c$-dual group. In this connection the physics literature considered reflection positive distributions $D \in \mathcal{S}^{\prime}$, invariant under the euclidean group. The Hilbert space $\mathcal{H}_{D} \subseteq \mathcal{S}^{\prime}$ specified by the corresponding positive definite kernel [61] leads to a reflection positive representation of the euclidean motion group [49,50]. This motivates our discussion of $G$-invariant gaussian measures corresponding to a unitary representation $(\pi, \mathcal{H})$ of $G$. The replacement for the Gelfand triple $\left(\mathcal{S}, L^{2}\left(\mathbb{R}^{n}\right), \mathcal{S}^{\prime}\right)$ is the Gelfand triple $\left(\mathcal{H}^{\infty}, \mathcal{H}, \mathcal{H}^{-\infty}\right)$, where $\mathcal{H}^{\infty}$ denotes the Fréchet space of smooth vectors in $\mathcal{H}$ and $\mathcal{H}^{-\infty}$ is it conjugate linear dual (the space of distribution vectors). Here the natural question is for which representations the gaussian measure of $\mathcal{H}$ can be realized on $\mathcal{H}^{-\infty}$ ? A partial answer is given in Theorem 5.17, Corollary 5.19, and Proposition 5.20. All of this links naturally to the theory of generalized Wiener spaces and white noise processes for $\mathbb{R}^{n}$, indexed by $L^{2}\left(\mathbb{R}^{n}\right)$; see, e.g., [2, 3, 5, 29].

Finally we would like to mention that there is also an important branch of applications of reflection positivity in statistical physics which does not refer to semigroups at all; see [19] and the more recent [51], where the corresponding group $G$ may be a torus, hence does not contain any proper open subsemigroup. We hope to develop this point in future work.

Our article is organized as follows. In the first section we start by recalling the basic facts about reflection positive Hilbert spaces and reflection positive representations [49, 50]. We always have a symmetric Lie group $(G, \tau)$ and a subsemigroup $S$ invariant under $s^{\#}=\tau(s)^{-1}$. Based on ideas from [42], we introduce the special class of reflection positive Hilbert spaces of Markov type. The main result is Proposition 2.9.

In Section 3 we discuss stochastic processes indexed by a symmetric Lie group $(G, \tau)$, where the forward time $\mathbb{R}_{+}$is replaced by a subsemigroup $S \subset G$ invariant under $s \mapsto s^{\sharp}=\tau(s)^{-1}$. Here we prove our generalization of the reconstruction theorem (Theorem 3.22) which reconstructs for a so-called positive semigroup structure a $(G, S, \tau)$-measure space from which it can be derived. From the representation theoretic perspective, it corresponds to finding euclidean realizations of unitary representations of the $c$-dual group $G^{c}$. Unfortunately, the reconstruction process requires that $G=S \cup S^{-1}$, a property which is briefly discussed in Section 3.5. 
In Section 4 we build a bridge between Markov processes and the reconstruction process. To this end, we return to the classical setting, where the symmetric Lie group is $\left(\mathbb{R}, \mathbb{R}_{+},-\mathrm{id}\right)$, but the measure space is the path space $P(Q)=Q^{\mathbb{R}}$ of a polish group $Q$ and the corresponding $\left(\mathbb{R}, \mathbb{R}_{+},-\mathrm{id}_{\mathbb{R}}\right)$-measure space is given by a one-parameter group $P_{t} f=f * \mu_{t}, t \geq 0$, of left invariant Markov kernels on some space $L^{2}(Q, \nu)$. Here our main result concerns a factorization of the measure space $P(Q)$ as $Q \times P_{*}(Q)$ and a corresponding factorization of the measure preserving action of $\mathbb{R}$. For the special case where $\mu_{t}$ is the gaussian semigroup on $\mathbb{R}^{d}$, the corresponding measure on $P_{*}\left(\mathbb{R}^{d}\right)$ is the Wiener measure, but the measure on $P\left(\mathbb{R}^{d}\right)$ is a product of Lebesgue and Wiener measure. The main results in Section 4 is Theorem 4.9 which relates all this to the reconstruction process and hence to reflection positive representations.

In Section 5 we discuss the second quantization functor and how it can be used to derive from an orthogonal reflection positive representation a gaussian $(G, S, \tau)$-probability spaces (Proposition 5.6). To understand the ambiguity in this construction we also discuss equivalence of gaussian measures for reproducing kernel Hilbert spaces, and in Subsection 5.4 we connect this issue to our previous work [49] on distributions on $G$ and distribution vectors of a unitary representation of $G$. In Theorem 5.17 we determine when a gaussian measure $\gamma_{\mathcal{H}}$ of a Hilbert space $\mathcal{H}$ can be realized in the dual of the space $\bigcap \mathcal{D}\left(A^{n}\right)$ of smooth vector of a self adjoint operator $A$. This is then applied to a unitary representation of $G$ in Corollary 5.19.

The article ends with two appendixes where needed material on stochastic processes and Markov semigroups is collected.

\section{Reflection positive representations}

In this preliminary section we collect some results and definitions from [49, 50] concerning reflection positive representations.

Definition 2.1. A reflection positive Hilbert space is a triple $\left(\mathcal{E}, \mathcal{E}_{+}, \theta\right)$, where $\mathcal{E}$ is a Hilbert space, $\theta$ a unitary involution and $\mathcal{E}_{+}$a closed subspace which is $\theta$-positive in the sense that the hermitian form $\langle v, w\rangle_{\theta}:=\langle\theta v, w\rangle$ is positive semidefinite on $\mathcal{E}_{+}$.

For a reflection positive Hilbert space $\left(\mathcal{E}, \mathcal{E}_{+}, \theta\right)$, let $\mathcal{N}:=\left\{u \in \mathcal{E}_{+}:\langle\theta u, u\rangle=0\right\}$ and let $\widehat{\mathcal{E}}$ be the completion of $\mathcal{E}_{+} / \mathcal{N}$ with respect to the inner product $\langle\cdot, \cdot\rangle_{\theta}$. Let $q: \mathcal{E}_{+} \rightarrow \widehat{\mathcal{E}}, v \mapsto q(v)=\widehat{v}$ be the canonical map. Then $\mathcal{E}_{+}^{\theta}:=\left\{v \in \mathcal{E}_{+}: \theta v=v\right\}$ is the maximal subspace of $\mathcal{E}_{+}$on which $q$ is isometric.

Definition 2.2. Let $\left(\mathcal{E}, \mathcal{E}_{+}, \theta\right)$ be a reflection positive Hilbert space. If $\mathcal{E}_{0} \subseteq \mathcal{E}_{+}^{\theta}$ is a closed subspace, $\mathcal{E}_{-}:=\theta\left(\mathcal{E}_{+}\right)$, and $E_{0}, E_{ \pm}$the orthogonal projections onto $\mathcal{E}_{0}$ and $\mathcal{E}_{ \pm}$, then we say that $\left(\mathcal{E}, \mathcal{E}_{0}, \mathcal{E}_{+}, \theta\right)$ is a reflection positive Hilbert space of Markov type if

$$
E_{+} E_{0} E_{-}=E_{+} E_{-} .
$$

Lemma 2.3. Suppose that $\mathcal{E}$ is a Hilbert space, $\theta: \mathcal{E} \rightarrow \mathcal{E}$ is a unitary involution, $\mathcal{E}_{+}$is a closed subspace and $\mathcal{E}_{0} \subseteq \mathcal{E}_{+}^{\theta}$. Let $\mathcal{E}_{-}=\theta\left(\mathcal{E}_{+}\right)$.

(i) If $\left(\mathcal{E}, \mathcal{E}_{0}, \mathcal{E}_{+}, \theta\right)$ satisfies the Markov condition (2.1), then $\mathcal{E}_{+}$is $\theta$-positive, $\mathcal{E}_{0}=\mathcal{E}_{+}^{\theta}, \mathcal{N}=$ $\mathcal{E}_{+} \ominus \mathcal{E}_{0}$ and $q: \mathcal{E}_{0} \rightarrow \widehat{\mathcal{E}}$ is a unitary isomorphism.

(ii) If $q_{0}: \mathcal{E}_{0} \rightarrow \widehat{\mathcal{E}}$ is a unitary isomorphism, then (2.1) holds.

Proof. (i) By definition, we have $\theta E_{+}=E_{-} \theta$ so $\mathcal{E}_{+}^{\theta} \subseteq \mathcal{E}_{+} \cap \mathcal{E}_{-}$. Let $u \in \mathcal{E}_{+}^{\theta} \ominus \mathcal{E}_{0}$. Then the operator on the right hand side of (2.1) reproduces $u$ while the left hand side yields 0 . Hence $u=0$ and $\mathcal{E}_{0}=\mathcal{E}_{+}^{\theta}$. As $\mathcal{E}_{0} \subset \mathcal{E}_{+}^{\theta}$ it follows that $E_{0} \theta=\theta E_{0}=E_{0}$ which implies that

$$
E_{+} \theta E_{+}=E_{+} E_{-} \theta=E_{+} E_{0} E_{-} \theta=E_{0} \theta E_{+}=E_{0} E_{+}=E_{0} .
$$


It follows that, for $u \in \mathcal{E}_{+}$, we have

$$
\langle\theta u, u\rangle=\left\langle E_{+} \theta E_{+} u, u\right\rangle=\left\langle E_{0} u, u\right\rangle=\left\|E_{0} u\right\|^{2} \geq 0
$$

We obtain in particular that $\|q(v)\|=\left\|E_{0} v\right\|$ for $v \in \mathcal{E}_{+}$, so that $\left.q\right|_{\mathcal{E}_{0}}: \mathcal{E}_{0} \rightarrow \widehat{\mathcal{E}}$ is a unitary isomorphism.

(ii) Since $\left.q\right|_{\mathcal{E}_{+}^{\theta}}$ is isometric, we obtain $\mathcal{E}_{0}=\mathcal{E}_{+}^{\theta}$ and thus $\mathcal{N}=\mathcal{E}_{+} \cap \theta\left(\mathcal{E}_{0}\right)^{\perp}=\mathcal{E}_{+} \ominus \mathcal{E}_{0}$. This leads to the orthogonal decomposition $\mathcal{E}_{+}+\mathcal{E}_{-}=\theta(\mathcal{N}) \oplus \mathcal{E}_{0} \oplus \mathcal{N}$ and to $\mathcal{E}_{0}=\mathcal{E}_{+} \cap \mathcal{E}_{-}$. Now (2.1) follows.

In the following, if $\left(\mathcal{E}, \mathcal{E}_{0}, \mathcal{E}_{+}, \theta\right)$ satisfies the Markov condition (2.1), then we will always assume that $\mathcal{E}_{0}=\mathcal{E}_{+}^{\theta}$ and hence only write $\left(\mathcal{E}, \mathcal{E}_{+}, \theta\right)$.

Definition 2.4. If $\tau$ is an involutive automorphism of the Lie group $G$, then we call $(G, \tau)$ a symmetric Lie group. A symmetric semigroup is a triple $(G, S, \tau)$, where $(G, \tau)$ is a symmetric Lie group and $S \subseteq G$ is a subsemigroup satisfying

(S1) $S$ is invariant under $s \mapsto s^{\sharp}=\tau(s)^{-1}$,

(S2) $H S=S$ for $H:=\left(G^{\tau}\right)_{0}$,

(S3) $\mathbf{1} \in \bar{S}$.

We define a left invariant partial order $\prec_{S}$ on $G$ by $g \prec_{S} h$ if $g^{-1} h \in S$, i.e., $h \in g S$.

Example 2.5. (1) $\left(\mathbb{R}, \mathbb{R}_{+},-\mathrm{id}_{\mathbb{R}}\right)$ and $\left(\mathbb{Z}, \mathbb{N}_{0},-\mathrm{id}_{\mathbb{Z}}\right)$ are the most elementary examples of symmetric semigroups.

(2) Semigroups with polar decomposition: Let $(G, \tau)$ be a symmetric Lie group and $H$ be an open subgroup of $G^{\tau}:=\{g \in G: \tau(g)=g\}$. We denote the derived involution $\mathfrak{g} \rightarrow \mathfrak{g}$ by the same letter and define $\mathfrak{h}=\{x \in \mathfrak{g}: \tau(x)=x\}=\mathfrak{g}^{\tau}$ and $\mathfrak{q}=\{x \in \mathfrak{g}: \tau(x)=-x\}=\mathfrak{g}^{-\tau}$. Then $\mathfrak{g}=\mathfrak{h} \oplus \mathfrak{q}$. We say that the open subsemigroup $S \subseteq G$ has a polar decomposition if there exists an $H$-invariant open convex cone $C \subset \mathfrak{q}$ containing no affine lines such that $S=H \exp C$ and the map $H \times C \rightarrow S, h, X \mapsto h \exp X$ is a diffeomorphism (cf. [26, 44, 48]). Typical examples are the complex Olshanski semigroups in complex simple Lie groups such as $\mathrm{SU}_{p, q}(\mathbb{C})_{\mathbb{C}} \cong \mathrm{SL}_{p+q}(\mathbb{C})$. They exist if and only if $G / K$ is a bounded symmetric domain. This is equivalent to the existence of a $G$-invariant convex cone $C \subset i \mathfrak{g}$ such that $G \exp C$ is a semigroup. More generally we have the causal symmetric spaces of non-compact type like $\mathrm{SO}_{o}(1, n+1) / \mathrm{SO}_{o}(1, n)$. In this case $\mathfrak{q} \simeq \mathbb{R}^{n+1}$ and $C$ can be taken as the open light-cone.

(3) The simply connected covering group $G:=\widetilde{\mathrm{SL}}_{2}(\mathbb{R})$ of $\mathrm{SL}_{2}(\mathbb{R})$ carries an involution $\tau$ acting on $\mathfrak{s l}_{2}(\mathbb{R})$ by

$$
\tau\left(\begin{array}{cc}
x & y \\
z & -x
\end{array}\right)=\left(\begin{array}{cc}
x & -y \\
-z & -x
\end{array}\right)
$$

and there exists a closed subsemigroup $S \subseteq G$ whose boundary is

$$
\partial S=H(S):=S \cap S^{-1}=\exp (\mathfrak{b}) \quad \text { with } \quad \mathfrak{b}:=\left\{\left(\begin{array}{cc}
x & y \\
0 & -x
\end{array}\right): x, y \in \mathbb{R}\right\} .
$$

This semigroup satisfies $S^{\sharp}=S$, the subgroup $H(S)$ is $\tau$-invariant, but strictly larger than $G_{0}^{\tau}$ (see also Section 3.5 for more on this semigroup).

Definition 2.6. For a symmetric semigroup $(G, S, \tau)$, a unitary representation $U$ of $G$ on $\left(\mathcal{E}, \mathcal{E}_{+}, \theta\right)$ is called reflection positive if $\theta U_{g} \theta=U_{\tau(g)}$ for $g \in G$ and $U_{s} \mathcal{E}_{+} \subseteq \mathcal{E}_{+}$for every $s \in S$. 
Remark 2.7. Let $G_{\tau}=G \rtimes\{1, \tau\}$. Then $\theta U_{g} \theta=U_{\tau(g)}$ holds for every $g \in G$ if and only if $U$ extends to a unitary representation of $G_{\tau}$ by defining $U_{\tau}=\theta$.

Remark 2.8. (a) If $\left(U_{g}\right)_{g \in G}$ is a reflection positive representation of $(G, S, \tau)$ on $\left(\mathcal{E}, \mathcal{E}_{+}, \theta\right)$, then we obtain contractions $\left(\widehat{U}_{s}\right)_{s \in S}$, on $\widehat{\mathcal{E}}$, determined by

$$
\widehat{U}_{s} \circ q=\left.q \circ U_{s}\right|_{\mathcal{E}_{+}},
$$

and this leads to an involutive representation $(\widehat{U}, \widehat{\mathcal{E}})$ of $S$ by contractions (cf. [35, Corollary 3.2] and [49]). We then call $\left(U, \mathcal{E}, \mathcal{E}_{+}, \theta\right)$ a euclidean realization of $(\widehat{U}, \widehat{\mathcal{E}})$. We refer to [46] and [45] for methods for obtaining a unitary representation of $G^{c}$ from $\widehat{U}$.

(b) For $(G, S, \tau)=\left(\mathbb{R}, \mathbb{R}_{+},-\mathrm{id}_{\mathbb{R}}\right)$, continuous reflection positive unitary one-parameter groups $\left(U_{t}\right)_{t \in \mathbb{R}}$ lead to a strongly continuous semigroup $(\widehat{U}, \widehat{\mathcal{E}})$ of hermitian contractions and every such semigroup $(C, \mathcal{H})$ has a natural euclidean realization obtained as the GNS representation associated to the positive definite operator-valued function $\varphi(t):=C_{|t|}, t \in \mathbb{R}[50$, Proposition 6.1].

The following proposition is a generalization of [50, Proposition 5.17] which applies to the special case $\left(\mathbb{R}, \mathbb{R}_{+},-\mathrm{id}_{\mathbb{R}}\right)$.

Proposition 2.9. Let $\left(U_{g}\right)_{g \in G}$ be a reflection positive unitary representation of $(G, S, \tau)$ on $\left(\mathcal{E}, \mathcal{E}_{+}, \theta\right)$, let $\mathcal{E}_{0} \subseteq\left(\mathcal{E}_{+}\right)^{\theta}$ be a subspace and $\Gamma=\left.q\right|_{\mathcal{E}_{0}}: \mathcal{E}_{0} \rightarrow \widehat{\mathcal{E}}$. If $\left(\mathcal{E}, \mathcal{E}_{0}, \mathcal{E}_{+}, \theta\right)$ is of Markov type, then the following assertions hold:

(i) The reflection positive function $\varphi: G \rightarrow B\left(\mathcal{E}_{0}\right), \varphi(g):=E_{0} U_{g} E_{0}$, is multiplicative on $S$.

(ii) $\varphi(s)=\Gamma^{*} \widehat{U}_{s} \Gamma$ for $s \in S$, i.e., $\Gamma$ intertwines $\left.\varphi\right|_{S}$ with $\widehat{U}$.

Proof. (i) Let $\mathcal{K} \subseteq \mathcal{H}$ be the $U$-invariant closed subspace generated by $\mathcal{E}_{0}$. Let $\left(\mathcal{E}_{0}\right)^{G}$ denote the linear space of all maps $G \rightarrow \mathcal{E}_{0}$. Then the map

$$
\Phi: \mathcal{K} \rightarrow\left(\mathcal{E}_{0}\right)^{G}, \quad \Phi(v)(g):=E_{0} U_{g} v
$$

is an equivalence of the representation $U$ of $G$ on $\mathcal{K}$ with the GNS representation defined by $\varphi$, and the representation $\widehat{U}$ of $S$ on $\widehat{\mathcal{E}}$ is equivalent to the GNS representation defined by $\left.\varphi\right|_{S}$, where the map $q: \mathcal{E}_{+} \rightarrow \widehat{\mathcal{E}}$ corresponds to the restriction $\left.f \mapsto f\right|_{S}$ [49, Proposition 1.11]. The inclusion $\iota: \mathcal{E}_{0} \hookrightarrow \mathcal{H}_{\varphi}$ is given by $\iota(v)(g)=E_{0} U_{g} v=\varphi(g) v$ for $g \in G$, and likewise the inclusion $\widehat{\iota}: \mathcal{E}_{0} \hookrightarrow \mathcal{H}_{\left.\varphi\right|_{S}}$ is given by $\iota(v)(s)=\varphi(s) v$ for $s \in S$. Lemma 2.3 implies the surjectivity of the inclusion $\widehat{\iota}$. In view of [50, Lemma 10.3], this is equivalent to the multiplicativity of $\left.\varphi\right|_{S}$.

(ii) If $\Gamma$ is unitary, then [50, Lemma 5.16(ii)] implies that $q=\left.\Gamma \circ E_{0}\right|_{\mathcal{E}_{+}}$. For $s \in S$, the relation $\widehat{U}_{s} \circ q=\left.q \circ U_{s}\right|_{\mathcal{E}_{+}}$leads to $\left.\widehat{U}_{s} \Gamma E_{0}\right|_{\mathcal{E}_{+}}=\left.\Gamma E_{0} U_{s}\right|_{\mathcal{E}_{+}}$, so that $\Gamma^{*} \widehat{U}_{s} \Gamma=E_{0} U_{s} E_{0}=\varphi(s)$, i.e., $\Gamma$ intertwines $\varphi(s)$ with $\widehat{U}_{s}$.

Lemma 2.10. Assume that $U$ is a reflection positive representation of $(G, S, \tau)$ on $\left(\mathcal{E}, \mathcal{E}_{+}, \theta\right)$. Let $\mathcal{E}_{0} \subseteq \mathcal{E}_{+}^{\theta}$. If $\varphi(g)=E_{0} U_{g} E_{0}$ is multiplicative and $\mathcal{E}_{0}$ is $S$-cyclic in $\mathcal{E}_{+}$then $\left(\mathcal{E}, \mathcal{E}_{+}, \theta\right)$ is of Markov type.

Proof. As in the proof of Lemma 2.3, this follows from [50, Lemma 10.3].

Remark 2.11. If $G=S \cup S^{-1}$ and $\mathcal{E}_{0}$ is $G$-cyclic in $\mathcal{E}$. Then $\mathcal{E}_{0}$ is $S$-cyclic in $\mathcal{E}_{+}$and $\mathcal{E}_{+}+\mathcal{E}_{-}$ is dense in $\mathcal{E}$, so that the Markov condition leads to $\mathcal{E}=\mathcal{N} \oplus \mathcal{E}_{0} \oplus \theta(\mathcal{N})$. 


\section{Reflection positive Lie group actions on measure spaces}

In this section we generalize several results from [41] and [42] to situations where $\left(\mathbb{R}, \mathbb{R}_{+},-\mathrm{id}_{\mathbb{R}}\right)$ is replaced by a symmetric semigroup $(G, S, \tau)$. This leads us to the concept of a $(G, S, \tau)$ measure space generalizing Klein's Osterwalder-Schrader path spaces for $\left(\mathbb{R}, \mathbb{R}_{+},-\mathrm{id}_{\mathbb{R}}\right)$. The Markov $(G, S, \tau)$-measure spaces generalize the path spaces studied by Klein and Landau in [42]. The main result of this section is the correspondence between $(G, S, \tau)$-measure spaces and the corresponding positive semigroup structures. For $(G, S, \tau)=\left(\mathbb{R}, \mathbb{R}_{+},-\mathrm{id}_{\mathbb{R}}\right)$ this has been developed in [41, 42], motivated by Nelson's work on the Feynman-Kac formula [54].

\section{$3.1(G, S, \tau)$-measure spaces}

In this section we introduce basic concepts related to $(G, S, \tau)$-measure spaces and draw some consequences from the definitions.

Definition 3.1. Let $(G, \tau)$ be a group with an involutive automorphism $\tau$. A $(G, \tau)$-measure space is a quadruple $\left((Q, \Sigma, \mu), \Sigma_{0}, U, \theta\right)$ consisting of the following ingredients:

(GP1) a measure space $(Q, \Sigma, \mu)$,

(GP2) a sub- $\sigma$-algebra $\Sigma_{0}$ of $\Sigma$,

(GP3) a measure preserving action $U: G \rightarrow \operatorname{Aut}(\mathcal{A})$ on the $W^{*}$-algebra $\mathcal{A}:=L^{\infty}(Q, \Sigma, \mu)$ which is strongly continuous in measure, i.e. ${ }^{1}$,

$$
\lim _{g \rightarrow \mathbf{1}} \mu\left(\left|U_{g} f-f\right| \geq \varepsilon\right)=0 \quad \text { for } \quad \varepsilon>0 \quad \text { and } \quad f \in \mathcal{A} \cap L^{1}(Q, \Sigma, \mu),
$$

and

(GP4) a measure preserving involutive automorphism $\theta$ of $L^{\infty}(Q, \Sigma, \mu)$ for which $\theta U_{g} \theta=U_{\tau(g)}$ for $g \in G$ and $\theta E_{0} \theta=E_{0}$, where $E_{0}: L^{\infty}(Q, \Sigma, \mu) \rightarrow L^{\infty}\left(Q, \Sigma_{0}, \mu\right)$ is the conditional expectation.

(GP5) $\Sigma$ is generated by the sub- $\sigma$-algebras $\Sigma_{g}:=U_{g} \Sigma_{0}, g \in G$.

If $\mu$ is a probability measure, we speak of a $(G, \tau)$-probability space. If $(G, S, \tau)$ is a symmetric semigroup, then we write $\Sigma_{ \pm}$for the sub- $\sigma$-algebra generated by $\left(\Sigma_{s}\right)_{s \in S^{ \pm 1}}$, and $E_{ \pm}$for the corresponding conditional expectations.

Definition 3.2. (a) A $(G, \tau)$-measure space is called reflection positive with respect to $S$ if

$$
\langle\theta f, f\rangle \geq 0 \quad \text { for } \quad f \in \mathcal{E}_{+}:=L^{2}\left(Q, \Sigma_{+}, \mu\right) .
$$

This is equivalent to $E_{+} \theta E_{+} \geq 0$ as an operator on $L^{2}(Q, \Sigma, \mu)$ and obviously implies $\theta E_{0}=E_{0}$. If this condition is satisfied and, in addition, $\Sigma_{0}$ is invariant under the group $H(S):=S \cap S^{-1}$, then we call it a $(G, S, \tau)$-measure space ${ }^{2}$.

(b) A Markov $(G, S, \tau)$-measure space is a $(G, S, \tau)$-measure space with the Markov property $E_{+} E_{-}=E_{+} E_{0} E_{-}$(cf. Definition 2.1(b)).

\footnotetext{
${ }^{1}$ Since the $G$-action on $\mathcal{A}$ is measure preserving, it defines natural representations on $L^{1}(Q, \Sigma, \mu)$ and on $L^{2}(Q, \Sigma, \mu)$. However, in general we do not have an action of $G$ on the set $Q$ itself, but $G$ acts naturally on the set $\Sigma / \sim$, where $A \sim B$ if $\mu(A \Delta B)=0$. This set corresponds to the idempotents in the algebra $\mathcal{A}$. The continuity condition (GP3) is equivalent to the continuity of the corresponding unitary representation of $G$ on $L^{2}(Q, \Sigma, \mu)$ which in turn is equivalent to $\mu(g E \Delta E) \rightarrow 0$ for $g \rightarrow \mathbf{1}$ and $E \in \Sigma$ with $\mu(E)<\infty$. See the discussion in Appendix A or [64, p. 107] for more details.

${ }^{2}$ Note that $E_{+} \theta E_{+} \geq 0$ is equivalent to the condition that the kernel $K(A, B):=\mu(A \cap \theta(B))$ on $\Sigma_{+}$is positive definite.
} 
Proposition 2.9 now implies:

Proposition 3.3. For any $(G, S, \tau)$-measure space $\left((Q, \Sigma, \mu), \Sigma_{0}, U, \theta\right)$, we put $\mathcal{E}:=L^{2}(Q, \Sigma, \mu)$, $\mathcal{E}_{0}:=L^{2}\left(Q, \Sigma_{0}, \mu\right)$ and $\mathcal{E}_{ \pm}:=L^{2}\left(Q, \Sigma_{ \pm}, \mu\right)$. Then the natural action of $G$ on $\mathcal{E}$ defines a reflection positive representation of $(G, S, \tau)$. The Markov property is equivalent to the natural map $\mathcal{E}_{0} \rightarrow \widehat{\mathcal{E}}$ being unitary and this implies that the function $\varphi: S \rightarrow B\left(\mathcal{E}_{0}\right), \varphi(s)=E_{0} U_{s} E_{0}$ is multiplicative.

Definition 3.4. (a) A positive semigroup structure $(\mathcal{H}, P, \mathcal{A}, \Omega)$ for a symmetric semigroup $(G, S, \tau)$ consists of

(PS1) a Hilbert space $\mathcal{H}$

(PS2) a strongly continuous $*$-representation $\left(P_{s}\right)_{s \in S}$ of $(S, \sharp)$ by contractions on $\mathcal{H}$,

(PS3) a commutative von Neumann algebra $\mathcal{A}$ on $\mathcal{H}$ normalized by the operators $\left(P_{h}\right)_{h \in H(S)}$, and

(PS4) a unit vector $\Omega \in \mathcal{H}$, such that

(a) $P_{s} \Omega=\Omega$ for every $s \in S$.

(b) $\Omega$ is cyclic for the (not necessarily selfadjoint) subalgebra $\mathcal{B} \subseteq B(\mathcal{H})$ generated by $\mathcal{A}$ and $\left\{P_{s}: s \in S\right\}$.

(c) For positive elements $A_{1}, \ldots, A_{n} \in \mathcal{A}$ and $s_{1}, \ldots, s_{n-1} \in S$, we have

$$
\left\langle A_{1} P_{s_{1}} A_{2} \cdots P_{s_{n-1}} A_{n} \Omega, \Omega\right\rangle \geq 0 .
$$

(b) A standard positive semigroup structure for a symmetric semigroup $(G, S, \tau)$ consists of a $\sigma$-finite measure space $(M, \mathfrak{S}, \nu)$ and

(SPS1) a representation $\left(P_{s}\right)_{s \in S}$ of $S$ on $L^{\infty}(M, \nu)$ by positivity preserving operators, i.e., $P_{s} f \geq 0$ for $f \geq 0$.

(SPS2) $P_{s} 1=1$ for $s \in S$ (the Markov condition).

(SPS3) $P$ is involutive with respect to $\nu$, i.e.,

$$
\int_{M} P_{s}(f) h d \nu=\int_{M} f P_{s^{\sharp}}(h) d \nu \quad \text { for } \quad s \in S, \quad f, h \geq 0 .
$$

(SPS4) $P$ is strongly continuous in measure, i.e., for each $f \in L^{1}(M, \nu) \cap L^{\infty}(M, \nu)$ and every $\delta>0, s_{0} \in S$, we have $\lim _{s \rightarrow s_{0}} \nu\left(\left\{\left|P_{s} f-P_{s_{0}} f\right| \geq \delta\right\}\right)=0$.

The preceding definition generalizes the corresponding classical concepts for the case $(G, S, \tau)$ $=\left(\mathbb{R}, \mathbb{R}_{+},-\operatorname{id}_{\mathbb{R}}\right)([41]$ for $(\mathrm{a})$ and $[42]$ for $(\mathrm{b}))$.

Remark 3.5. (a) For an operator $P$ on $L^{2}(X, \mu)$, the condition to be positivity preserving does not imply that $P$ is symmetric. In fact, if $(P f)(x)=f(T x)$ for some measure preserving map $T: X \rightarrow X$, then $P$ is positivity preserving and isometric. Hence we cannot expect $P$ to be symmetric if $T$ is not an involution.

(b) Applying (SPS3) with $h=1$, we obtain $\int_{M} P_{s}(f) d \mu=\int_{M} f d \mu$, i.e., that the measure $\mu$ is invariant under the operators $\left(P_{s}\right)_{s \in S}$.

(c) If $P: M \times \mathfrak{S} \rightarrow[0, \infty]$ is a Markov kernel (cf. Appendix B), then

$$
(P f)(x):=\int_{M} P(x, d y) f(y)
$$

defines a positivity preserving operator on $L^{\infty}(M, \mathfrak{S}, \nu)$ satisfying $P 1=1$. 
In view of [7, Lemma 36.2], kernels on $M \times \mathfrak{S}$ are in one-to-one correspondence with additive, positively homogeneous maps $T$ from the convex cone of non-negative measurable functions $M \rightarrow[0, \infty]$ into itself which are Daniell continuous in the sense that $\lim _{n \rightarrow \infty} T\left(f_{n}\right)=T\left(\lim _{n \rightarrow \infty} f_{n}\right)$ for monotone sequences $f_{n} \leq f_{n+1} \cdot^{3}$

Remark 3.6. We consider a standard positive semigroup structure for $(G, S, \tau)$.

(a) Conditions (SPS1/2) imply that $\left\|P_{s}\right\| \leq 1$ on $L^{\infty}(M, \nu)$. Further (SPS2/3) imply that the restriction of $P_{s}$ to $L^{1} \cap L^{\infty}$ is measure preserving, and from [42, Proposition 1.2(i)] it follows that $P_{s^{\sharp} s}$ defines a contraction on $L^{2}(M, \nu)$. This implies that we obtain a $*$-representation of $(S, \sharp)$ on $L^{2}(M, \nu)$. The proof of [42, Proposition 1.2(ii)] further shows that (SPS4) implies that this representation is strongly continuous because we have for $f \in L^{1} \cap L^{2}$ :

$$
\begin{aligned}
\left\|P_{s} f-P_{s_{0}} f\right\|_{2}^{2} & \leq \int_{\left\{\left|P_{s} f-P_{s_{0}} f\right| \geq \varepsilon\right\}}\left|P_{s} f-P_{s_{0}} f\right|^{2} d \nu+\int_{\left\{\left|P_{s} f-P_{s_{0}} f\right| \leq \varepsilon\right\}}\left|P_{s} f-P_{s_{0}} f\right|^{2} d \nu \\
& \leq\left(2\|f\|_{\infty}\right)^{2} \nu\left(\left\{\left|P_{s} f-P_{s_{0}} f\right| \geq \varepsilon\right\}\right)+2 \varepsilon\|f\|_{1} .
\end{aligned}
$$

For $h \in H(S)$, both operators $P_{h}$ and $P_{h^{-1}}=P_{h}^{-1}$ are positivity preserving and fix 1 , and since $L^{\infty}(M, \nu)$ is a commutative von Neumann algebra, they are algebra automorphisms by [12, Theorem 3.2.3]. The relation $P_{h}(f g)=P_{h}(f) P_{h}(g)$ then leads to

$$
P_{h} M_{f} P_{h}^{-1}=M_{P_{h}(f)}
$$

for the multiplication operators $M_{f}$. In particular, the action of $H(S)$ on $L^{2}(M, \nu)$ normalizes $L^{\infty}(M, \nu)$.

(b) If $\nu$ is a probability measure, $\Omega:=1$ and $\mathcal{A}:=L^{\infty}(M, \nu)$, then the preceding discussion shows that we also have a positive semigroup structure in the sense of Definition 3.4(a) for which 1 is a cyclic vector for $\mathcal{A}$. Note that Definition 3.4(b) is not a special case of (a) because it does not require the measure $\nu$ to be finite.

The following proposition shows that the requirement that $\Omega$ is cyclic for $\mathcal{A}$ describes those positive semigroup structures which are standard.

Proposition 3.7 ([41, Proposition 3.5]). Let $(M, \mathfrak{S}, \nu)$ be a probability space and $\left(P_{s}\right)_{s \in S}$ be a positivity preserving continuous *-representation of $S$ by contractions on $L^{2}(M, \nu)$, i.e.,

$$
P_{s} 1=1 \quad \text { and } \quad P_{s} f \geq 0 \quad \text { for } \quad f \geq 0, \quad s \in S .
$$

Then $\left(L^{2}(M), Q, L^{\infty}(M), 1\right)$ is a positive semigroup structure for which 1 is a cyclic vector for $L^{\infty}(M)$.

Conversely, let $(\mathcal{H}, P, \mathcal{A}, \Omega)$ be a positive semigroup structure for which $\Omega$ is a cyclic vector for $\mathcal{A}$. Then there exists a probability space $M$ and a positivity preserving semigroup $\widetilde{Q}$ on $L^{2}(M)$ such that $(\mathcal{H}, P, \mathcal{A}, \Omega) \cong\left(L^{2}(M), \widetilde{Q}, L^{\infty}(M), 1\right)$ as positive semigroup structures.

Proof. The first part is an immediate consequence of the definitions (see also Remark 3.6(a)), so we only have to prove the second statement. So let $(\mathcal{H}, P, \mathcal{A}, \Omega)$ be a positive semigroup structure for which $\Omega$ is a cyclic vector for $\mathcal{A}$. Let $M$ be a compact space with $\mathcal{A} \cong C(M)$. Then we obtain on $M$ a probability measure $\nu$, defined by

$$
\int_{M} f(x) d \nu(x)=\langle f \Omega, \Omega\rangle \quad \text { for } \quad f \in \mathcal{A} .
$$

\footnotetext{
${ }^{3}$ The notion of a positivity preserving operator on $L^{\infty}(M, \mathfrak{S}, \nu)$ is slightly weaker than this concept. In particular, it only operates on equivalence classes of functions in $L^{\infty}(X, \mathfrak{S}, \nu)$ and not on functions itself. In concrete situations, the positivity preserving operators actually come from kernels, which makes them easier to deal with.
} 
Since $\Omega$ is $\mathcal{A}$-cyclic, the map $C(M) \rightarrow \mathcal{H}, f \mapsto f \Omega$ extends to a unitary map $L^{2}(M, \nu) \rightarrow \mathcal{H}$ intertwining $\mathcal{A}$ with the von Neumann algebra $L^{\infty}(M, \nu)$. It is clear that (SPS1-3) are satisfied and Lemma A.1 implies the continuity in measure.

Example 3.8 (the real oscillator semigroup). We consider the Hilbert space $\mathcal{H}=L^{2}\left(\mathbb{R}^{d}\right.$ ), with respect to Lebesgue measure.

(a) On $\mathcal{H}$ we have a unitary representation by the group $\mathrm{GL}_{d}(\mathbb{R})$ by

$$
\left(T_{h} f\right)(x):=|\operatorname{det}(h)|^{-d / 2} f\left(h^{-1} x\right) \quad \text { for } \quad h \in \mathrm{GL}_{d}(\mathbb{R}), \quad x \in \mathbb{R}^{d},
$$

and we also have two representations of the abelian semigroup $\operatorname{Sym}_{d}(\mathbb{R})_{+}$(the convex cone of positive semidefinite matrices):

(a) Each $A \in \operatorname{Sym}_{d}(\mathbb{R})_{+}$defines a multiplication operator $\left(M_{A} f\right)(x):=e^{-\langle A x, x\rangle} f(x)$ which is positivity preserving on $L^{\infty}\left(\mathbb{R}^{n}\right)$ but does not preserve 1 ; it preserves the Dirac measure $\delta_{0}$ in the origin.

(b) Each $A \in \operatorname{Sym}_{d}(\mathbb{R})_{+}$specifies a uniquely determined (possibly degenerate) gaussian measure $\mu_{A}$ on $\mathbb{R}^{d}$ whose Fourier transform is given by $\widehat{\mu}_{A}(x)=e^{-\langle A x, x\rangle / 2}$. Then the convolution operator $C_{A} f:=f * \mu_{A}$ is positivity preserving and leaves Lebesgue measure on $\mathbb{R}^{d}$ invariant. For $A=\mathbf{1}$, we thus obtain the heat semigroup as $\left(\mu_{t \mathbf{1}}\right)_{t \geq 0}$.

Any composition of these 3 types of operators $T_{h}, M_{A}$ and $C_{A}$ is positivity preserving on $L^{\infty}\left(\mathbb{R}^{d}\right)$, and they generate a $*$-representation of the Olshanski semigroup $S:=H \exp (C)$ in the symmetric Lie group $G:=\operatorname{Sp}_{2 d}(\mathbb{R})$, where $H=\mathrm{GL}_{d}(\mathbb{R}), C=\operatorname{Sym}_{d}(\mathbb{R})_{+} \times \operatorname{Sym}_{d}(\mathbb{R})_{+} \subseteq$ $\operatorname{Sym}_{d}(\mathbb{R})^{2}=\mathfrak{q}$, and

$$
\tau\left(\begin{array}{cc}
A & B \\
C & -A^{\top}
\end{array}\right)=\left(\begin{array}{cc}
A & -B \\
-C & -A^{\top}
\end{array}\right) \quad \text { for } \quad\left(\begin{array}{cc}
A & B \\
C & -A^{\top}
\end{array}\right) \in \mathfrak{s p}_{2 d}(\mathbb{R})
$$

with $B^{\top}=B, C^{\top}=C$ (cf. Examples 2.5). The real Olshanski semigroup $S$ is the fixed point set an antiholomorphic involutive automorphism of the so-called oscillator semigroup $S_{\mathbb{C}}=$ $G^{c} \exp (W)$ which is a complex Olshanskii semigroup [24, 30]. The elements in the interior of $S$ act on $L^{2}\left(\mathbb{R}^{n}\right)$ by kernel operators with positive gaussian kernels and the elements of $S_{\mathbb{C}}$ correspond to complex-valued gaussian kernels. The semigroup $S$ contains many interesting symmetric one-parameter semigroups such as the Mehler semigroup $e^{-t H_{\text {osc }}}$ generated by the oscillator Hamiltonian

$$
H_{\mathrm{osc}}=-\sum_{j=1}^{n} \partial_{j}^{2}+\frac{1}{4} \sum_{j=1}^{n} x_{j}^{2}-\frac{d}{2} \mathbf{1},
$$

which fixes the Gaussian $e^{-\|x\|^{2} / 4}$.

(b) The parabolic subgroup $P:=\operatorname{Sym}_{d}(\mathbb{R}) \rtimes \mathrm{GL}_{d}(\mathbb{R})_{+} \subseteq \mathrm{Sp}_{2 d}(\mathbb{R})$ is also a symmetric Lie subgroup with $\mathfrak{h}=\mathfrak{g l}_{d}(\mathbb{R})$ and $\mathfrak{q} \cong \operatorname{Sym}_{d}(\mathbb{R})$. Here the commutative von Neumann algebra $\mathcal{A}=L^{\infty}\left(\mathbb{R}^{d}\right)$ is invariant under conjugation with the operators $T_{h}$, so that $(A, h) \mapsto C_{A} T_{h}$ defines a $*$-representation of the semigroup $S:=\operatorname{Sym}_{d}(\mathbb{R})_{+} \rtimes H$ that leads to a standard positive semigroup structure on $L^{2}\left(\mathbb{R}^{d}, d x\right)$.

The preceding example naturally extends to infinite-dimensional spaces as follows.

Example 3.9. Let $\mathcal{H}$ be a real Hilbert space.

(a) In Lemma 5.5 below we show that every continuous $*$-representation of a topological involutive semigroup $(S, \sharp)$ by contractions on a real Hilbert space $\mathcal{H}$ defines a standard positive semigroup structure on the gaussian probability space $\left(\mathcal{H}^{a}, \gamma_{\mathcal{H}}\right)$. Here $\mathcal{H}^{a}$ is the algebraic dual 
space of $\mathcal{H}$ and $\gamma_{\mathcal{H}}$ is the unique probability measure with Fourier transform $\widehat{\gamma}_{\mathcal{H}}(v)=e^{-\|v\|^{2} / 2}$ (Example 5.3).

(b) Some of the structure from Example 3.8 extends to infinite-dimensional Hilbert spaces $\mathcal{H}$. For the multiplication operator $M_{A}$ to be non-zero, one has to require that $A \geq 0$ is trace class (cf. [68, p. 153]). Likewise, the measures $\mu_{A}$ exist if $A$ is trace class. In an orthonormal basis $\left(e_{n}\right)_{n \in \mathbb{N}}$ in which $A$ is diagonal with $A e_{n}=a_{n} e_{n}$, the measure $\mu_{A}$ is $\otimes_{n=1}^{\infty} \gamma_{a_{n} t}$, where $d \gamma_{t}(x)=(2 \pi t)^{-1 / 2} e^{-x^{2} / 2 t} d x$ are the centered gaussian measures on $\mathbb{R}$. For a linear operator $h \in \mathrm{GL}(\mathcal{H})$ to act on $L^{2}\left(\mathcal{H}^{a}, \gamma_{\mathcal{H}}\right)$, it is necessary and sufficient that $g^{\top} g-\mathbf{1}$ is Hilbert-Schmidt, i.e., that the polar decomposition has the form $g=u e^{X}$ with $\|X\|_{2}<\infty$ (see also Theorem 5.7 and [52] for more details).

(c) For $\mathcal{H}=\mathbb{R}^{d}$, we have $d \gamma(x):=d \gamma_{\mathcal{H}}(x)=(2 \pi)^{-d / 2} e^{-\frac{\|x\|^{2}}{2}} d x$ (Example 5.4), we obtain in particular a standard positive semigroup structure for the contraction semigroup

$$
\mathcal{C}\left(\mathbb{R}^{d}\right):=\left\{g \in \mathrm{GL}_{d}(\mathbb{R}):\|g\| \leq 1\right\}=\mathrm{O}_{d}(\mathbb{R}) \exp \left(-\operatorname{Sym}_{d}(\mathbb{R})_{+}\right),
$$

where $\operatorname{Sym}_{d}(\mathbb{R})_{+}$denotes the closed convex cone of positive semidefinite symmetric $(d \times d)$ matrices. The corresponding Markov operators are given by $(\Gamma(h) f)(x)=f\left(h^{-1} x\right)$ for $h \in$ $\mathrm{O}_{d}(\mathbb{R})$. Since every element $s \in \mathcal{C}\left(\mathbb{R}^{d}\right)$ has a polar decomposition $s=h \exp (-X), X^{\top}=X \geq 0$, diagonalization of $X$ reduces the description of the corresponding operator to the case $d=1$. For $0<c<1$ we have

$$
(\Gamma(c) f)(x)=\int_{\mathbb{R}^{d}} \widetilde{K}_{c}(x, y) f(y) d \gamma(y) \quad \text { with } \quad \widetilde{K}_{c}(x, y)=\left(1-c^{2}\right)^{-d / 2} e^{\frac{\|y\|^{2}}{2}} e^{-\frac{\|c x-y\|^{2}}{2\left(1-c^{2}\right)}}
$$

(cf. [55, p. 218]). For $c=e^{-t}, t \geq 0$, we thus obtain the Mehler semigroup, also called the Ornstein-Uhlenbeck semigroup. It can also be described by the Mehler formula [11, Section 1.4]

$$
(\Gamma(c) f)(x)=\int_{\mathbb{R}^{d}} f\left(c x+\sqrt{1-c^{2}} y\right) d \gamma(y) .
$$

These operators form a hermitian strongly continuous contraction semigroup for which $\gamma$ is the unique invariant probability measure. In view of [11, Section 2.9], (3.2) still holds for infinitedimensional spaces.

(d) For $\mathcal{H}=\mathbb{R}^{d}$, it is instructive to connect the gaussian picture to the Lebesgue picture. To this end, we note that

$$
\Phi: \quad L^{2}\left(\mathbb{R}^{d}\right) \rightarrow L^{2}\left(\mathbb{R}^{d}, \gamma\right), \quad \Phi(f)(x)=(2 \pi)^{d / 4} e^{\|x\|^{2} / 4} f(x)
$$

is a unitary isomorphism. Conjugating with $\Phi$, we therefore obtain a $*$-representation $\pi$ of the real Olshanski semigroup $S$ (Example 3.8) on $L^{2}\left(\mathbb{R}^{d}, \gamma\right)$. This transformation has no effect on the multiplication operators $M_{A}$ because they commute with $\Phi$ but it transforms the convolution operators $C_{A}$ into more complicated Markov operators. For instance the Laplace operator $\Delta=\sum_{j} \partial_{j}^{2}$ transforms into

$$
\Delta^{\prime}:=\Phi \Delta \Phi^{-1}=\Delta+\frac{\|x\|^{2}}{4}-\left(E+\frac{d}{2} \mathbf{1}\right), \quad \text { where } \quad E=\sum_{j} x_{j} \partial_{j}
$$

is the Euler operator. The unitary representation of $H=\mathrm{GL}_{d}(\mathbb{R})$ transforms into

$$
T_{h}^{\prime} f=e^{\frac{1}{4}\left(\|x\|^{2}-\left\|h^{-1} x\right\|^{2}\right)}\left(T_{h} f\right)(x)=|\operatorname{det} h|^{-d / 2} e^{\frac{1}{4}\left(\|x\|^{2}-\left\|h^{-1} x\right\|^{2}\right)} f\left(h^{-1} x\right) .
$$

As the operators $\Phi$ and $\Phi^{-1}$ are positivity preserving, the semigroup $S$ also acts by positivity preserving operators on $L^{2}\left(\mathbb{R}^{n}, \gamma\right)$. If $s \in S$ preserves 1 , then the transformed operator $s^{\prime}$ preserves the Gaussian $e^{\|x\|^{2} / 4}$. 
(e) The oscillator Hamiltonian $H_{\text {osc }}$ from (3.1) transforms into

$$
-\Delta^{\prime}:=\Phi H_{\mathrm{osc}} \Phi^{-1}=\Phi \circ\left(-\Delta+\frac{\|x\|^{2}}{4}-\frac{d}{2} \mathbf{1}\right) \circ \Phi^{-1}=E-\Delta=\sum_{j} x_{j} \partial_{j}-\partial_{j}^{2},
$$

which also makes sense in the infinite-dimensional case, where $\Delta^{\prime}$ is called Umemura's LaplaceBeltrami operator [23, p. 221]. It is the infinitesimal generator of the Mehler semigroup $e^{t \Delta^{\prime}}$.

Example 3.10 (a finite-dimensional example). (a) We consider the finite set $M=\{1, \ldots, n\}$, endowed with the counting measure, so that $L^{2}(M, \nu) \cong \mathbb{R}^{d}$. Let $S \subseteq \mathrm{GL}_{n}(\mathbb{R})$ be the subsemigroup of invertible doubly stochastic matrices $S=\left(S_{i j}\right)$, i.e., $\sum_{i} S_{i j}=\sum_{j} S_{i j}=1$ for all $i, j$. According to the Birkhoff-von Neumann theorem, $S \subseteq \operatorname{conv}(H)$, where $H=S \cap S^{-1} \cong S_{n}$ is the group of permutation matrices. Then $S \subseteq \mathrm{GL}_{n}(\mathbb{R})$ a transposition stable subsemigroup for which the action on $\mathbb{R}^{n} \cong L^{\infty}(M)$ by matrix multiplication defines a standard positive semigroup structure.

(b) A natural generalization of the preceding example is obtained by starting with a probability space $(Q, \Sigma, \nu)$ and the group $H:=\operatorname{Aut}(Q, \Sigma, \nu)$ of measure preserving automorphisms of $(Q, \Sigma)$. Then the probability measures $\mu$ on $Q$ defining by $P_{\mu}(f)(x):=\int_{H} f\left(h^{-1} x\right) d \mu(h)$ invertible operators on $L^{\infty}(Q, \Sigma, \nu)$ naturally generalizes the semigroup $S \subseteq \mathrm{GL}_{d}(\mathbb{R})$ from (a).

\subsection{Stochastic processes indexed by Lie groups}

We now introduce the concept of stochastic processes where the more common index set $\mathbb{R}$, thought of as a time, is replaced by a Lie group. The forward direction is then given by a semigroup contained in $G$.

Definition 3.11. Let $(Q, \Sigma, \mu)$ be a probability space. A stochastic process indexed by a group $G$ is a family $\left(X_{g}\right)_{g \in G}$ of measurable functions $X_{g}: Q \rightarrow(B, \mathfrak{B})$, where $(B, \mathfrak{B})$ is a measurable space, called the state space of the process.

For any such process, we obtain a measurable map $\Phi: Q \rightarrow B^{G}, \Phi(q)=\left(X_{g}(q)\right)_{g \in G}$ with respect to the product $\sigma$-algebra $\mathfrak{B}^{G}$. Then $\nu:=\Phi_{*} \mu$ is a measure on $B^{G}$, called the distribution of the process $\left(X_{g}\right)_{g \in G}$. This measure is uniquely determined by the measures $\nu_{\mathbf{g}}$ on $G^{n}$, obtained for any tuple $\mathbf{g}:=\left(g_{1}, \ldots, g_{n}\right) \in G^{n}$ as the image of $\mu$ under the map

$$
X_{\mathrm{g}}=\left(X_{g_{1}}, \ldots, X_{g_{n}}\right): Q \rightarrow B^{n}
$$

(cf. [23, Section 1.3]). If $\mathbf{g}=(g)$ for some $g \in G$ then we write $\nu_{g}$ for $\nu_{\mathbf{g}}$.

The process $\left(X_{g}\right)_{g \in G}$ is called stationary if the corresponding distribution on $B^{G}$ is invariant under the translations

$$
\left(U_{g} \nu\right)_{h}:=\nu_{g^{-1} h} \quad \text { for } \quad g, h \in G \text {. }
$$

If $\tau \in \operatorname{Aut}(G)$ is an automorphism, then we call the process $\tau$-invariant if the distribution is invariant under

$$
(\tau \nu)_{h}:=\nu_{\tau^{-1}(h)} \quad \text { for } \quad h \in G
$$

The following definition generalizes some concepts from $G=\mathbb{R}$ to general topological groups (cf. [40, Section 2]). We only formulate the definition for real valued functions, but will use without further comments the complex analogue where in the $L^{2}$-inner products the second factor has to be conjugated. 
Definition 3.12. (a) A linear stochastic process (indexed by the group $G$ ) is a stochastic process $(\varphi(v, g))_{v \in V, g \in G}$, where $V$ is a real linear space, $\varphi$ is linear in $v$ and each $\varphi(v, g)$ is a real valued measurable function on a fixed probability space $(Q, \Sigma, \mu)$.

(b) A linear stochastic process is said to be of second order if each $\varphi(v, g)$ is square integrable and the stochastic process is full in the sense that, up to sets of measure zero, $\Sigma$ is the smallest $\sigma$-algebra for which all functions $\varphi(v, g)$ are measurable.

(c) That $\varphi$ is continuous in the quadratic mean means that $V$ is a topological vector space, $\varphi$ is of second order and $\varphi: V \times G \rightarrow L^{2}(Q, \Sigma, \mu)$ is continuous.

(d) Assume that $\varphi$ is of second order. Then $\varphi$ is (wide sense) stationary means that the kernel

$$
K\left((v, g),\left(v^{\prime}, g^{\prime}\right)\right):=\mathbb{E}\left(\overline{\varphi(v, g)} \varphi\left(v^{\prime}, g^{\prime}\right)\right)=\int_{Q} \overline{\varphi(v, g)} \varphi\left(v^{\prime}, g^{\prime}\right) d \mu
$$

on $V \times G$ satisfies the invariance condition

$$
K\left((v, g),\left(v^{\prime}, g^{\prime}\right)\right)=K\left(\left(v, g_{0} g\right),\left(v^{\prime}, g_{0} g^{\prime}\right)\right) \quad \text { for } \quad g_{0}, g, g^{\prime} \in G, \quad v, v^{\prime} \in V .
$$

Let $\varphi$ be a stochastic process. To adapt better to the discussion in the previous section, we assume that $\varphi(v, g)$ is complex-valued and that $V$ is a complex vector space. The real case can be treated in the same way. If $\varphi$ is a linear stochastic process of second order, then the square integrability of the functions $\varphi(v, g)$ implies that the covariance kernel

$$
K\left((v, g),\left(v^{\prime}, g^{\prime}\right)\right):=\mathbb{E}\left(\overline{\varphi(v, g)} \varphi\left(v^{\prime}, g^{\prime}\right)\right)=\int_{Q} \overline{\varphi(v, g)} \varphi\left(v^{\prime}, g^{\prime}\right) d \mu
$$

is defined. It is a positive definite kernel on $V \times G$, and the map

$$
\Phi: \quad L^{2}(Q, \Sigma, \mu) \rightarrow \mathcal{H}_{K} \subseteq \mathbb{C}^{V \times G}, \quad \Phi(F)(v, g):=\langle F, \varphi(v, g)\rangle
$$

is a partial isometry of $L^{2}(Q, \Sigma, \mu)$ onto the reproducing kernel Hilbert space $\mathcal{H}_{K} \subseteq \mathbb{C}^{V \times G}$. Its adjoint $\Phi^{*}$ is an isometric embedding of $\mathcal{H}_{K}$ whose range is the closed subspace on $L^{2}(Q, \Sigma, \mu)$

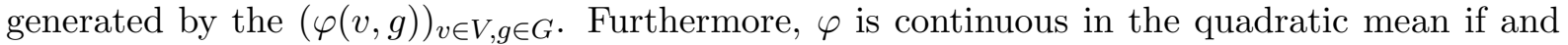
only if $K$ is continuous.

We will from now on assume that $\varphi$ is continuous in the quadratic mean. Then

$$
\left(v, v^{\prime}\right):=\mathbb{E}\left(\varphi(v, \mathbf{1}) \overline{\varphi\left(v^{\prime}, \mathbf{1}\right)}\right)=\int_{Q} \varphi(v, \mathbf{1}) \overline{\varphi\left(v^{\prime}, \mathbf{1}\right)} d \mu
$$

defines a continuous positive semidefinite form on $V$, and in the following we may therefore replace $V$ by the complex Hilbert space $\mathcal{F}$ obtained by completing the quotient

$$
V /\{v \in V:(v, v)=0\}
$$

with respect to the induced norm. We thus obtain a stochastic process $\varphi(w, g)$ on $\mathcal{F} \times G$. Now we may consider $\mathcal{F}$ as a closed subspace of $L^{2}(Q, \Sigma, \mu)$ and write $E_{0}: L^{2}(Q, \Sigma, \mu) \rightarrow \mathcal{F}$ for the orthogonal projection.

If $K$ is continuous and $\mathcal{F}$ is a complex Hilbert space, then $K$ also defines a positive definite $B(\mathcal{F})$-valued kernel

$$
K^{\mathcal{F}}: G \times G \rightarrow B(\mathcal{F}), \quad\left\langle K^{\mathcal{F}}(g, h) v, w\right\rangle=K((v, h),(w, g)),
$$

so that we can realize the closed subspace of $L^{2}(Q, \mu)$ generated by the $\varphi(v, g)$ as the reproducing kernel Hilbert space $\mathcal{H}_{K^{\mathcal{F}}} \subseteq \mathbb{C}^{\mathcal{F}}$ of $\mathcal{F}$-valued functions on $G$. Here $\mathcal{F}$ is isomorphic to the closed subspace of $L^{2}(Q, \Sigma, \mu)$ generated by the functions $(\varphi(v, \mathbf{1}))_{v \in V}$. 
Consider now (d) and assume that $\varphi$ is continuous in the quadratic mean. This condition ensures that we obtain a unitary representation $\left(U_{g}\right)_{g \in G}$ on the subspace $\mathcal{H}_{K} \subseteq L^{2}(Q, \mu)$ satisfying

$$
\left(U_{g} \varphi\right)(v, h)=\varphi(v, g h), \quad v \in V, \quad g, h \in G .
$$

Under assumption (c), we thus obtain a strongly continuous $B(\mathcal{F})$-valued positive definite function $r(g):=E_{0} U_{g} E_{0}$. The invariance condition leads to a $\operatorname{Sesq}(V)$-valued positive definite function on $G$ as discussed in [51], but we will not follow up on that in this article.

Example 3.13. Let $(G, \tau)$ be a symmetric Lie group and $\left(X_{g}\right)_{g \in G}$ be a stationary, $\tau$-invariant, full stochastic process on $\left(Q, \Sigma_{Q}, \mu_{Q}\right)$. Then its distribution $\left(B^{G}, \mathfrak{B}^{G}, \nu\right)$ satisfies the conditions $(\mathrm{GP} 1,2,4,5)$ of a $(G, \tau)$-probability space with respect to the natural actions of $G$ and $\tau$ on $B^{G}$, where $\Sigma_{0}$ is the $\sigma$-algebra generated by $\left(X_{h}\right)_{h \in H}$, i.e., the smallest subalgebra for which these functions are measurable. In this context (GP3) is equivalent to the continuity of the unitary representation of $G$ on $L^{2}\left(B^{G}, \mathfrak{B}^{G}, \nu\right)$. We refer to Appendix A for a more detailed discussion of the continuity condition.

Example 3.14 (cf. Proposition 3.3). Suppose that $\left((Q, \Sigma, \mu), \Sigma_{0}, U, \theta\right)$ is a $(G, \tau)$-probability space, so that we obtain on $\mathcal{E}:=L^{2}(Q, \Sigma, \mu)$ a unitary representation of $G$. The subspace $\mathcal{E}_{0}:=L^{2}\left(Q, \Sigma_{0}, \mu\right)$ is cyclic under the algebra $\mathcal{B}$ generated by $\left(U_{g}\right)_{g \in G}$ and the multiplication operators $M_{f}, f \in L^{\infty}\left(Q, \Sigma_{0}, \mu\right)$. The prescription

$$
\varphi(v, g):=\pi(g) v, \quad g \in G, \quad v \in \mathcal{E}_{0}
$$

defines a wide sense stationary process indexed by $\mathcal{E}_{0} \times G$.

The scalar product on $L^{2}(Q, \Sigma, \mu)$ is completely determined by the $n$-point functionals:

$$
\left\langle U_{g_{1}} M_{f_{1}} \cdots M_{f_{n-1}} U_{g_{n}} M_{f_{n}} v, w\right\rangle_{\mathcal{E}_{0}}, \quad v, w \in \mathcal{E}_{0}, \quad g_{j} \in G, \quad f_{j} \in L^{\infty}\left(Q, \Sigma_{0}, \mu\right),
$$

resp.,

$$
\left\langle U_{g_{1}} M_{f_{1}} \cdots U_{g_{n}} f_{n}, f_{0}\right\rangle_{\mathcal{E}_{0}}=\mathbb{E}\left(f_{0} \varphi\left(f_{1}, g_{1}\right) \varphi\left(f_{2}, g_{1} g_{2}\right) \cdots \varphi\left(f_{n}, g_{1} \cdots g_{n}\right) \mid \Sigma_{0}\right),
$$

or the operators

$$
E_{0} U_{g_{1}} M_{f_{1}} \cdots M_{f_{n-1}} U_{g_{n}} E_{0}, \quad g_{j} \in G, \quad f_{j} \in L^{\infty}\left(Q, \Sigma_{0}, \mu\right) .
$$

In general $\mathcal{E}_{0}$ is not cyclic for $G$, so the process $\varphi(v, g)$ provides a means to construct the whole space $L^{2}(Q, \Sigma, \mu)$ in the spirit of a GNS construction for $\mathcal{B}$.

Example 3.15. In [64] free euclidean fields (scalar of positive mass $m>0$ ) are dealt with as gaussian processes indexed by the Hilbert space $N_{m}$ defined by the norm

$$
\|f\|_{N_{m}}^{2}=2 \int_{\mathbb{R}^{d+1}} \frac{|\widehat{f}(k)|^{2}}{k^{2}+m^{2}} d^{2} k \quad \text { for } \quad f \in \mathcal{S}\left(\mathbb{R}^{d+1}\right),
$$

so that the corresponding euclidean Hilbert space is the Fock space $\Gamma\left(N_{m}\right)$ (see Section 5.1). Here $N_{m}$ carries a unitary representation of the euclidean group $E(d)=\operatorname{Mot}\left(\mathbb{R}^{d}\right)$, and since the time translation group has continuous spectrum, the corresponding action on $\Gamma\left(N_{m}\right)$ is ergodic by Theorem 5.26 below (for time translations, space translations and the full euclidean group).

[64, Theorem III.6] provides a Feynman-Kac-Nelson formula for the gaussian free field. In this context, Nelson's main achievement in [54] was that he obtained a manifestly euclidean invariant path integral. 


\subsection{Associated positive semigroup structures and reconstruction}

Our goal in this section is to prove the reconstruction theorem (Theorem 3.18). This is a central result which asserts that positive semigroup structures all come from $(G, S, \tau)$-measure spaces.

We start with the following lemma which is an adaption of [41, Lemma 2.2] and [42, Corollary 1.5] to $(G, S, \tau)$.

Lemma 3.16. Let $\left((Q, \Sigma, \mu), \Sigma_{0}, U, \theta\right)$ be a $(G, S, \tau)$-measure space, $\mathcal{A}:=L^{\infty}\left(Q, \Sigma_{0}, \mu\right), \mathcal{E}:=$ $L^{2}(Q, \Sigma, \mu), \mathcal{E}_{0}:=L^{2}\left(Q, \Sigma_{0}, \mu\right)$, and $q: \mathcal{E}_{+}:=L^{2}\left(Q, \Sigma_{+}, \mu\right) \rightarrow \widehat{\mathcal{E}}$ be the canonical map. Then the following assertions hold:

(a) For $f \in \mathcal{A}$, let $M_{f}$ denote the corresponding multiplication operator on $\mathcal{E}$. Then there exists a bounded operator $\widehat{M}_{f} \in B(\widehat{\mathcal{E}})$ with $\left.q \circ M_{f}\right|_{\mathcal{E}_{+}}=\widehat{M}_{f} \circ q$ and $\left\|\widehat{M}_{f}\right\|=\|f\|_{\infty}$.

(b) $\pi(f):=\widehat{M}_{f}$ is a faithful weakly continuous representation of the commutative von Neumann algebra $\mathcal{A}$ on $\widehat{\mathcal{E}}$.

(c) In the Markov case we identify $\widehat{\mathcal{E}}$ with $\mathcal{E}_{0}$ and $q$ with $E_{0}$ (Proposition 3.3). For $g_{1} \prec_{S}$ $g_{2} \prec_{S} \cdots \prec_{S} g_{n}$ in $G$, non-negative functions $f_{1}, \ldots, f_{n} \in \mathcal{A}$ and $f_{g_{j}}:=U_{g_{j}} f_{j}$, we have

$$
\int_{Q} f_{g_{1}} \cdots f_{g_{n}} d \mu=\int_{Q} \widehat{M}_{f_{1}} \widehat{U}_{g_{1}^{-1} g_{2}} \cdots \widehat{M}_{f_{n-1}} \widehat{U}_{g_{n-1}^{-1} g_{n}} \widehat{M}_{f_{n}} 1 d \mu .
$$

If, in addition, $\mu$ is finite and positive, then $\Omega:=\mu(Q)^{-1 / 2} q(1)$ satisfies:

(d) For $g_{1} \prec_{S} g_{2} \prec_{S} \cdots \prec_{S} g_{n}$ in $G, f_{1}, \ldots, f_{n} \in \mathcal{A}$ and $f_{g_{j}}:=U_{g_{j}} f_{j}$, we have

$$
\int_{Q} f_{g_{1}} \cdots f_{g_{n}} d \mu=\left\langle\widehat{M}_{f_{1}} \widehat{U}_{g_{1}^{-1} g_{2}} \cdots \widehat{M}_{f_{n-1}} \widehat{U}_{g_{n-1}^{-1} g_{n}} \widehat{M}_{f_{n}} \Omega, \Omega\right\rangle .
$$

(e) $\Omega$ is a separating vector for $\mathcal{A}$ and $\widehat{U}_{s} \Omega=\Omega$ for every $s \in S$.

(f) $\Omega$ is cyclic for the algebra $\mathcal{B}$ generated by $\mathcal{A}$ and $\left(\widehat{U}_{s}\right)_{s \in S}$.

Proof. (a, b) Since $\theta$ commutes with $E_{0}$, it preserves the subspace $\mathcal{E}_{0}:=L^{2}\left(Q, \Sigma_{0}, \mu\right)$. Reflection positivity and $\mathcal{E}_{0} \subseteq \mathcal{E}_{+}$now imply that $\theta(f)=f$ for $f \in \mathcal{E}_{0}$. The preceding argument implies that $M_{f}$ commutes with $\theta$. As $M_{f}$ and its adjoint $M_{f}^{*}=M_{\bar{f}}$ preserve $\mathcal{E}_{ \pm}$, these operators also preserve $\mathcal{N}:=\mathcal{E}_{+} \cap \theta\left(\mathcal{E}_{-}\right)^{\perp}$, so that we obtain a well-defined operator $\widehat{M}_{f}: q\left(\mathcal{E}_{+}\right) \rightarrow \widehat{\mathcal{E}}$. For $v \in \mathcal{E}_{+}$, we then have

$$
\left\|\widehat{M}_{f} \widehat{v}\right\|^{2}=\left\langle\theta M_{f} v, M_{f} v\right\rangle=\left\langle\theta M_{|f|^{2}} v, v\right\rangle \leq\|f\|_{\infty}^{2}\|v\|^{2} .
$$

Applying this estimate to the functions $\left(f^{n}\right)_{n \in \mathbb{N}_{0}}$, we get $\left\|\widehat{M}_{f}\right\| \leq\|f\|_{\infty}$ from [48, Lemma II.3.8]. Since $q$ is isometric on $\mathcal{E}_{0}$ and $\left\|\left.M_{f}\right|_{\mathcal{E}_{0}}\right\|=\|f\|_{\infty}$, we actually obtain the equality $\left\|\widehat{M}_{f}\right\|=\|f\|_{\infty}$. This proves (a) and (b).

(c) ([42, Corollary 1.5]) First we observe that the operator

$$
M_{f_{g_{1}}} \cdots M_{f_{g_{n}}} U_{g_{n}}=M_{U_{g_{1}} f_{1}} \cdots M_{U_{g_{n}} f_{n}} U_{g_{n}}=U_{g_{1}} M_{f_{1}} U_{g_{1}^{-1} g_{2}} M_{f_{2}} \cdots M_{f_{n-1}} U_{g_{n-1}^{-1} g_{n}} M_{f_{n}}
$$

on $\mathcal{E}_{+}$is intertwined by $q$ with $\widehat{U}_{g_{1}} \widehat{M}_{f_{1}} \widehat{U}_{g_{1}^{-1} g_{2}} \cdots \widehat{U}_{g_{n-1}^{-1} g_{n}} \widehat{M}_{f_{n}}$. Applying this operator to the constant function 1 and integrating yields the assertion.

From now on we assume that $0<\mu(Q)<\infty$. 
(d) With the same argument as under (c), we derive

$$
\begin{aligned}
\int_{Q} f_{g_{1}} \cdots f_{g_{n}} d \mu & =\left\langle M_{f_{g_{1}}} \cdots M_{f_{g_{n}}} 1,1\right\rangle \\
& =\left\langle\theta M_{f_{g_{1}}} \cdots M_{f_{g_{n}}} U_{g_{n}} 1,1\right\rangle=\left\langle\widehat{M}_{f_{1}} \widehat{U}_{g_{1}^{-1} g_{2}} \cdots \widehat{M}_{f_{n-1}} \widehat{U}_{g_{n-1}^{-1} g_{n}} \widehat{M}_{f_{n}} \Omega, \Omega\right\rangle .
\end{aligned}
$$

(e) The relation $\widehat{U}_{s} \Omega=\Omega$ is a trivial consequence of $U_{s} 1=1$ for $s \in S$. Since $\left.q\right|_{\mathcal{E}_{0}}$ is isometric and intertwines the representation of $\mathcal{A}$ on $\mathcal{E}_{0}$ with the representation of $\mathcal{A}$ on $\widehat{\mathcal{E}}$, the $\mathcal{A}$-separating vector $1 \in \mathcal{E}_{0}$ is mapped by $q$ to an $\mathcal{A}$-separating vector.

(f) Since $\mathcal{E}_{0}$ is $U_{S}$-cyclic in $\mathcal{E}_{+}$, this follows from the fact that 1 is $\mathcal{A}$-cyclic in $L^{2}\left(Q, \Sigma_{0}, \mu\right)$.

Definition 3.17. The preceding lemma shows that, if $\left((Q, \Sigma, \mu), \Sigma_{0}, U, \theta\right)$ is a finite $(G, S, \tau)$ measure space, then $(\widehat{\mathcal{E}}, \widehat{U}, \mathcal{A}, q(1))$ is a positive semigroup structure for

$$
\mathcal{A}=\left\{\widehat{M}_{f}: f \in L^{\infty}\left(Q, \Sigma_{0}, \mu\right)\right\}
$$

We call it the associated positive semigroup structure.

We now come to our version of Klein's reconstruction theorem. Note that every discrete group is in particular a 0-dimensional Lie group, so that the following theorem applies in particular to discrete groups.

Theorem 3.18 (Reconstruction Theorem). Let $(G, \tau)$ be a symmetric Lie group and $S \subseteq G$ be $a \sharp$-invariant subsemigroup satisfying $G=S \cup S^{-1}$. Then every positive semigroup structure for $(G, S, \tau)$ is associated to some $(G, S, \tau)$-probability space $\left((Q, \Sigma, \mu), \Sigma_{0}, U, \theta\right)$.

Proof. We follow the proof for $(G, S, \tau)=\left(\mathbb{R}, \mathbb{R}_{+},-\right.$id) in [41, Theorem 2.4]. In view of the Gelfand representation theorem, there exists a compact space $Q_{0}$ with $\mathcal{A} \cong C\left(Q_{0}\right)$ as $C^{*}$ algebras. Then $Q:=Q_{0}^{G}$, the space of all functions $\omega: G \rightarrow Q_{0}$, is compact with respect to the product topology. The group $G$ acts on $Q$ by

$$
\left(U_{g} \omega\right)(x):=\omega\left(g^{-1} x\right)
$$

and, accordingly, on functions on $Q$ by $\left(U_{g} F\right)(\omega)=F\left(U_{g^{-1}} \omega\right)$. We further obtain an involution $(\theta \omega)(g):=\omega(\tau(g))$ satisfying

$$
\theta U_{g} \theta=U_{\tau(g)} \quad \text { for } \quad g \in G .
$$

Let $\Sigma_{0}$ be the smallest $\sigma$-algebra on $Q$ for which $X_{\mathbf{1}}(\omega):=\omega(\mathbf{1})$ is measurable, $\Sigma_{g}:=U_{g} \Sigma_{\mathbf{1}}$, and let $\Sigma$ be the $\sigma$-algebra generated by the $\Sigma_{g}, g \in G$. To construct a measure $\mu$ on $\Sigma$, it suffices to construct a Radon measure on $Q$, i.e., a positive functional on the $C^{*}$-algebra $C(Q)$. By continuity, it actually suffices to do this on a dense unital subalgebra. Such a subalgebra is generated by the $G$-translates of functions in $\Xi:=C\left(Q_{0},\{0,1\}\right)$, which is a generating subset of $\mathcal{A}$. Now let $\mathcal{R} \subseteq C(Q)$ be the subspace spanned by functions of the form

$$
F(\omega):=\chi_{1}\left(\omega\left(g_{1}\right)\right) \cdots \chi_{n}\left(\omega\left(g_{n}\right)\right), \quad n \in \mathbb{N}_{0}, \quad \chi_{j} \in \Xi, \quad g_{j} \in G, \quad \text { i.e., } \quad F=\prod_{j=1}^{n} U_{g_{j}} \chi_{j},
$$

when we identify $C\left(Q_{0}\right)$ with the subalgebra $X_{1}^{*} C\left(Q_{0}\right)$ of $C(Q)$. Clearly, $\mathcal{R}$ is a subalgebra of $C(Q)$, and the Stone-Weierstraß theorem implies that it is dense.

Since $G=S \cup S^{-1}$, every finite subset of $G$ is of the form $\left\{g_{1}, \ldots, g_{n}\right\}$ with $g_{1} \prec_{S} \ldots \prec_{S} g_{n}$, i.e., $s_{j}:=g_{j}^{-1} g_{j+1} \in S$ for $j=1, \ldots, n-1$. Next we show that that, for $\left(A_{j}, g_{j}\right) \in \mathcal{A} \times G$ with $g_{1} \prec_{S} \cdots \prec_{S} g_{n}$, the operator

$$
A_{1} P_{s_{1}} A_{2} \cdots P_{s_{n-1}} A_{n}
$$


does not depend on the way we enumerate the $g_{j}$. Suppose that we also have $g_{j+1} \prec_{S} g_{j}$ for some $j$. Then $h:=s_{j}=g_{j}^{-1} g_{j+1} \in H(S)=S \cap S^{-1}$. We put $\widetilde{g}_{i}:=g_{i}$ for $i \neq j, j+1$ and set $\widetilde{g}_{j}:=g_{j+1}, \widetilde{g}_{j+1}=g_{j}$. Accordingly, we put $\widetilde{A}_{j}:=A_{j+1}$ and $\widetilde{A}_{j+1}:=A_{j}$. This leads to

$$
\widetilde{s}_{i}= \begin{cases}s_{i} & \text { for } i \neq j-1, j, j+1, \\ s_{j-1} s_{j}=s_{j-1} h & \text { for } i=j-1, \\ h^{-1} & \text { for } i=j, \\ s_{j} s_{j+1}=h s_{j+1} & \text { for } i=j+1 .\end{cases}
$$

We thus obtain

$$
\begin{aligned}
P_{\widetilde{s}_{j-1}} \widetilde{A}_{j} P_{\widetilde{s}_{j}} \widetilde{A}_{j+1} P_{\widetilde{s}_{j+1}} & =P_{s_{j-1} h} A_{j+1} P_{h}^{-1} A_{j} P_{h s_{j+1}}=P_{s_{j-1}}\left(P_{h} A_{j+1} P_{h}^{-1}\right) A_{j} P_{h} P_{s_{j+1}} \\
& =P_{s_{j-1}} A_{j}\left(P_{h} A_{j+1} P_{h}^{-1}\right) P_{h} P_{s_{j+1}}=P_{s_{j-1}} A_{j} P_{s_{j}} A_{j+1} P_{s_{j+1}}
\end{aligned}
$$

where we use that $P_{h} A_{j+1} P_{h}^{-1} \in \mathcal{A}, H(S)$ normalizes $\mathcal{A}$, and the commutativity of $\mathcal{A}$. The above argument shows the asserted independence of (3.3) because any increasing rearrangement can be obtained by successive transpositions.

For $n \in \mathbb{N}$ and $s_{1}, \ldots, s_{n-1} \in S$, the map

$$
\mathcal{A}^{n} \cong C\left(Q_{0}\right)^{n} \rightarrow \mathbb{R}, \quad\left(A_{1}, \ldots, A_{n}\right) \mapsto\left\langle A_{1} P_{s_{1}} A_{2} \cdots P_{s_{n-1}} A_{n} \Omega, \Omega\right\rangle
$$

is $n$-linear, so that there exists a well-defined linear functional $\rho: \mathcal{R} \rightarrow \mathbb{R}$ satisfying

$$
\rho\left(\prod_{j=1}^{n} U_{g_{j}} f_{j}\right)=\left\langle f_{1} P_{g_{1}^{-1} g_{2}} f_{2} \cdots P_{g_{n-1}^{-1} g_{n}} f_{n} \Omega, \Omega\right\rangle
$$

for $f_{1}, \ldots, f_{n} \in \operatorname{span} \Xi, g_{1} \prec_{S} \cdots \prec_{S} g_{n}, n \in \mathbb{N}_{0}$.

To see that $\rho$ is positive, we observe that every non-negative function $F \in \mathcal{R}$ can be written in the form

$$
F=\sum_{k} c_{k} F_{k} \quad \text { with } \quad F_{k}:=\prod_{j=1}^{n} U_{g_{j}} \chi_{j, k}, \quad \text { where } \quad F_{k} F_{k^{\prime}}=0 \quad \text { for } \quad k \neq k^{\prime} .
$$

Then $c_{k} \geq 0$ for all $k$, so that

$$
\rho(F)=\sum_{k} c_{k} \rho\left(\prod_{j=1}^{n} U_{g_{j, k}} \chi_{j, k}\right) \geq 0
$$

by (3.4). This proves that $\rho \geq 0$, and thus, by continuity, it extends to a positive functional on $C(Q)$, which defines a Radon measure $\mu$ on $Q$.

Next we show that $\left((Q, \Sigma, \mu), \Sigma_{0}, P, \theta\right)$ is a $(G, S, \tau)$-measure space. The $G$-invariance of the right hand side of (3.4) implies that $G$ acts on $Q$ by measure preserving transformations. To see that $\mu$ is also $\theta$-invariant, we first note that $g \prec_{S} h$ implies $\tau(h) \prec_{S} \tau(g)$ because $\tau(S)=S^{-1}$. We thus obtain

$$
\theta\left(\prod_{j=1}^{n} U_{g_{j}} f_{j}\right)=\prod_{j=0}^{n-1} U_{\tau\left(g_{n-j}\right)} f_{n-j} .
$$

Hence the $\theta$-invariance of $\mu$ follows from

$$
\begin{aligned}
\left\langle f_{1} P_{g_{1}^{-1} g_{2}} f_{2} \cdots P_{g_{n-1}^{-1} g_{n}} f_{n} \Omega, \Omega\right\rangle & =\left\langle\Omega, f_{n} P_{\tau\left(g_{n}\right)^{-1} \tau\left(g_{n-1}\right)} \cdots f_{2} P_{\tau\left(g_{2}\right)^{-1} \tau\left(g_{1}\right)} f_{1} \Omega\right\rangle \\
& =\left\langle f_{n} P_{\tau\left(g_{n}\right)^{-1} \tau\left(g_{n-1}\right)} \cdots f_{2} P_{\tau\left(g_{2}\right)^{-1} \tau\left(g_{1}\right)} f_{1} \Omega, \Omega\right\rangle .
\end{aligned}
$$


It remains to verify $\langle\theta F, F\rangle \geq 0$ for $F \in L^{2}\left(Q, \Sigma_{+}, \mu\right)$. The functions of the form

$$
f_{g_{1}} \cdots f_{g_{n}}=U_{g_{1}} M_{f_{1}} U_{g_{1}^{-1} g_{2}} \cdots U_{g_{n-1}^{-1} g_{n}} M_{f_{n}} U_{g_{n}}^{-1} 1, \quad f_{j} \in \mathcal{A}, \mathbf{1} \prec_{S} g_{1} \prec_{S} g_{2} \prec_{S} \cdots \prec_{S} g_{n},
$$

form a total subset of $\mathcal{E}_{+}$. On pairs of such functions we have

$$
\begin{aligned}
& \left\langle\theta f_{g_{1}} \cdots f_{g_{n}}, f_{h_{1}}^{\prime} \cdots f_{h_{m}}^{\prime}\right\rangle \\
& \quad=\left\langle\theta U_{g_{1}} M_{f_{1}} U_{g_{1}^{-1} g_{2}} \cdots U_{g_{n-1}^{-1} g_{n}} f_{n}, U_{h_{1}} M_{f_{1}^{\prime}} U_{h_{1}^{-1} h_{2}} \cdots U_{h_{m-1}^{-1} h_{m}} f_{m}^{\prime}\right\rangle \\
& \quad=\left\langle U_{\tau\left(g_{1}\right)} M_{f_{1}} U_{\tau\left(g_{1}\right)^{-1} \tau\left(g_{2}\right)} \cdots U_{\tau\left(g_{n-1}\right)^{-1} \tau\left(g_{n}\right)} f_{n}, U_{h_{1}} M_{f_{1}^{\prime}} U_{h_{1}^{-1} h_{2}} \cdots U_{h_{m-1}^{-1} h_{m}} f_{m}^{\prime}\right\rangle \\
& \quad=\left\langle 1, M_{f_{n}} U_{\tau\left(g_{n}\right)^{-1} \tau\left(g_{n-1}\right)} \cdots U_{\tau\left(g_{2}\right)^{-1} \tau\left(g_{1}\right)} M_{f_{1}} U_{\tau\left(g_{1}\right)^{-1}} U_{h_{1}} M_{f_{1}^{\prime}} U_{h_{1}^{-1} h_{2}} \cdots U_{h_{m-1}^{-1} h_{m}} f_{m}^{\prime}\right\rangle \\
& \quad=\left\langle\Omega, \widehat{M}_{f_{n}} P_{\tau\left(g_{n}\right)^{-1} \tau\left(g_{n-1}\right)} \cdots P_{\tau\left(g_{2}\right)^{-1} \tau\left(g_{1}\right)} \widehat{M}_{f_{1}} P_{\tau\left(g_{1}\right)^{-1}} P_{h_{1}} \widehat{M}_{f_{1}^{\prime}} P_{h_{1}^{-1} h_{2}} \cdots P_{h_{m-1}^{-1} h_{m}} \widehat{M}_{f_{m}^{\prime}} \Omega\right\rangle \\
& \quad=\left\langle P_{g_{1}} \widehat{M}_{f_{1}} P_{g_{1}^{-1} g_{2}} \cdots P_{g_{n-1}^{-1} g_{n}} \widehat{M}_{f_{n}} \Omega, P_{h_{1}} \widehat{M}_{f_{1}^{\prime}} P_{h_{1}^{-1} h_{2}} \cdots P_{h_{m-1}^{-1} h_{m}} \widehat{M}_{f_{m}^{\prime}} \Omega\right\rangle .
\end{aligned}
$$

This implies that

$$
\begin{aligned}
& \left\langle\theta \sum_{j} c_{j} f_{g_{1, j}} \cdots f_{g_{n, j}}, \sum_{k} c_{k} f_{g_{1, k}} \cdots f_{g_{n, k}}\right\rangle \\
& =\left\|\sum_{j} c_{j} P_{g_{1, j}} \widehat{M}_{f_{1, j}} P_{g_{1, j}^{-1} g_{2, j}} \cdots P_{g_{n-1, j}^{-1} g_{n, j}} \widehat{M}_{f_{n, j}} \Omega\right\|^{2} \geq 0 .
\end{aligned}
$$

We conclude that $\mathcal{E}_{+}$is $\theta$-positive and that the canonical map $q: \mathcal{E}_{+} \rightarrow \widehat{\mathcal{E}}$ is equivalent to the map

$$
q: \mathcal{E}_{+} \rightarrow \mathcal{H}, \quad f_{g_{1}} \cdots f_{g_{n}} \mapsto P_{g_{1}} \widehat{M}_{f_{1}} P_{g_{1}^{-1} g_{2}} \cdots P_{g_{n-1}^{-1} g_{n}} \widehat{M}_{f_{n}} \Omega
$$

This map satisfies $q \circ M_{f}=\widehat{M}_{f} \circ q$ and $q \circ U_{s}=P_{s} \circ q$ for $s \in S$. Therefore $(\mathcal{H}, P, \mathcal{A}, \Omega)$ is equivalent to the associated positive semigroup structure.

Remark 3.19. Theorem 3.18 implies in particular that, for every positive semigroup structure, $\Omega$ is separating for $\mathcal{A}$ [41, Remark 2.5].

Remark 3.20. We have seen in the proof of Theorem 3.18 that it is sometimes more convenient to deal with the unital $W^{*}$-algebra $\mathcal{A}=L^{\infty}\left(Q, \Sigma_{0}, \nu\right)$ as a $C^{*}$-algebra and write it as $C\left(Q_{0}\right)$ for a compact (Hausdorff) space $Q_{0}$. Then the Riesz representation theorem asserts that positive functionals on $C\left(Q_{0}\right)$ correspond to regular Borel measures $\mu$ on $Q_{0}$. On the other hand, the restriction of any such measure to the $\sigma$-algebra of Baire sets, i.e., the smallest $\sigma$-algebra for which all continuous functions are measurable, already determines the corresponding linear functional on $C\left(Q_{0}\right)$ by integration. Therefore the positive functionals on $C\left(Q_{0}\right)$ are in one-toone correspondence with finite positive Baire measures on $Q_{0}$.

Since the set $\Sigma_{0} / J_{\nu}$ of equivalence classes modulo $\nu$-null sets corresponds to the idempotents in $\mathcal{A}$, resp., $C\left(Q_{0}\right)$, Baire measures on $Q_{0}$ correspond to measures on $\left(Q, \Sigma_{0}\right)$ that are absolutely continuous with respect to $\nu$.

A slight variation of the argument in the proof of Theorem 3.18 leads to the following generalization of the Klein-Landau reconstruction theorem [42, Theorem 1.8] which deals with standard positive semigroup structures. For the proof of this theorem, we need a suitable version of [42, Lemma 1.9] to deal with the case where the measure $\nu$ is infinite. 
Lemma 3.21. Let $(G, S, \tau)$ be a symmetric semigroup with $G=S \cup S^{-1}$. Let $Q_{0}$ be a compact Hausdorff space and $\nu_{0}$ a $\sigma$-finite measure on the Baire sets in $Q_{0}$. Suppose that $\left(P_{s}\right)_{s \in S}$ is a positivity preserving semigroup acting on $C\left(Q_{0}\right)$ leaving the function 1 invariant. Then there exists a unique $\sigma$-finite Baire measure $\mu$ on the compact Hausdorff space $Q=Q_{0}^{G}$, such that, for

$$
\tau\left(s_{m}\right) \prec_{S} \cdots \prec_{S} \tau\left(s_{1}\right) \prec_{S} \mathbf{1} \prec_{S} t_{1} \prec_{S} \cdots \prec_{S} t_{n}
$$

and non-negative $g_{1}, \ldots, g_{m}, f_{1}, \ldots, f_{n} \in C\left(Q_{0}\right)$, we have

$$
\begin{aligned}
& \int_{Q} g_{m}\left(\varphi\left(\tau\left(s_{m}\right)\right)\right) \cdots g_{1}\left(\varphi\left(\tau\left(s_{1}\right)\right) f_{1}\left(\varphi\left(t_{1}\right)\right) \cdots f_{n}\left(\varphi\left(t_{n}\right)\right) d \mu(\varphi)\right. \\
& =\int_{Q_{0}}\left(P_{s_{1}} M_{g_{1}} P_{s_{1}^{-1} s_{2}} M_{g_{2}} \cdots P_{s_{m-1}^{-1} s_{m}} M_{g_{m}} 1\right)\left(P_{t_{1}} M_{f_{1}} P_{t_{1}^{-1} t_{2}} M_{f_{2}} \cdots P_{t_{n-1}^{-1} t_{n}} M_{f_{n}} 1\right) d \nu\left(q_{0}\right),
\end{aligned}
$$

where, as before, $M_{f}$ denotes multiplication by $f$.

Proof. First we assume that the measure $\nu$ is finite. Let $\mathcal{R} \subseteq C(Q)$ be as in the proof of Theorem 3.18. We then obtain a positive functional $\rho$ on $\mathcal{R}$, which is uniquely determined by the requirement that, for

$$
F(q)=g_{m}\left(\varphi\left(\tau\left(s_{m}\right)\right)\right) \cdots g_{1}\left(\varphi\left(\tau\left(s_{1}\right)\right)\right) f_{1}\left(\varphi\left(t_{1}\right)\right) \cdots f_{n}\left(\varphi\left(t_{n}\right)\right)
$$

we have

$$
\rho(F)=\int_{Q_{0}}\left(P_{s_{1}} M_{g_{1}} P_{s_{1}^{-1} s_{2}} M_{g_{2}} \cdots P_{s_{m-1}^{-1} s_{m}} M_{g_{m}} 1\right)\left(P_{t_{1}} M_{f_{1}} P_{t_{1}^{-1} t_{2}} M_{f_{2}} \cdots P_{t_{n-1}^{-1} t_{n}} M_{f_{n}} 1\right) d \nu\left(q_{0}\right)
$$

(see the proof of [42, Lemma 1.9] for details). The Riesz-Markov theorem now leads to a unique finite Baire measure $\mu$ on $Q$ with $\int_{Q} F d \mu=\rho(F)$ for $F \in \mathcal{R}$.

If $\nu$ is not finite, we write it as $\nu=\sum_{j} \nu_{j}$, where the measures $\nu_{j}$ are finite. If $\mu_{j}$ denotes the measure on $Q$ corresponding to $\nu_{j}$, the measure $\mu:=\sum_{j} \mu_{j}$ satisfies all our requirements.

Theorem 3.22 (Reconstruction Theorem - Standard Case). If $(G, S, \tau)$ is a symmetric semigroup with $G=S \cup S^{-1}$, then every standard positive semigroup structure for $(G, S, \tau)$ is associated to some $(G, S, \tau)$-measure space $\left((Q, \Sigma, \mu), \Sigma_{0}, U, \theta\right)$ which is unique up to $G$-equivariant isomorphism of measure spaces.

Proof. First we show uniqueness. Let $\left(\left(Q^{\prime}, \Sigma^{\prime}, \mu^{\prime}\right), \Sigma_{0}^{\prime}, U^{\prime}, \theta^{\prime}\right)$ be a Markov $(G, S, \tau)$-measure space for which $\left(L^{2}(M, \nu), P, L^{\infty}(M, \nu)\right)$ is the associated standard positive semigroup structure.

Lemma 3.16(c) implies that the integrals of products of the form $f_{g_{1}} \cdots f_{g_{n}}, g_{1} \prec_{S} \cdots \prec_{S} g_{n}$ are uniquely determined by the relation

$$
\int_{Q} f_{g_{1}} \cdots f_{g_{n}} d \mu^{\prime}=\int_{Q} M_{f_{1}} P_{g_{1}^{-1} g_{2}} \cdots M_{f_{n-1}} P_{g_{n-1}^{-1} g_{n}} M_{f_{n}} 1 d \nu
$$

Since such products for $f_{j} \in L^{2}(M, \nu) \cap L^{\infty}(M, \nu)$ form a total subset of $L^{2}\left(Q^{\prime}, \Sigma^{\prime}, \mu^{\prime}\right)$, any isomorphism $L^{2}\left(Q^{\prime}, \Sigma_{0}^{\prime}, \mu^{\prime}\right) \rightarrow L^{2}(M, \nu) \cong L^{2}\left(Q, \Sigma_{0}, \mu\right)$ extends to a $G$-equivariant isomorphism $L^{2}\left(Q^{\prime}, \Sigma^{\prime}, \mu^{\prime}\right) \rightarrow L^{2}(Q, \Sigma, \mu)$.

For the existence of the measure $\mu$, we now use Lemma 3.21. Here we write $Q_{0}$ for the spectrum of the $C^{*}$-algebra $\mathcal{A}:=L^{\infty}(M, \nu)$, so that $\mathcal{A} \cong C\left(Q_{0}\right)$ and $\nu$ defines a Baire measure on $Q_{0}$. We may then identify $(M, \mathfrak{S}, \nu)$ with $\left(Q_{0}, \Sigma_{0}, \nu\right)$, where $\Sigma_{0}$ is the $\sigma$-algebra of Baire subsets of $Q_{0}$. 
Now let $\mu$ be the Baire measure on $Q:=Q_{0}^{G}$ from Lemma 3.21. Then the relation $P_{s}^{*}=P_{s^{\sharp}}$, as operators on $L^{2}(M, \nu)$, leads to

$$
\int_{Q} f_{g_{1}} \cdots f_{g_{n}} d \mu=\int_{Q} M_{f_{1}} P_{g_{1}^{-1} g_{2}} \cdots M_{f_{n-1}} P_{g_{n-1}^{-1} g_{n}} M_{f_{n}} 1 d \nu
$$

for $0 \leq f_{1}, \ldots, f_{n} \in C\left(Q_{0}\right)$ and $g_{1} \prec_{S} \cdots \prec_{S} g_{n}$. The same calculations as in the proof of Theorem 3.18 now show that $\mu$ is invariant under $G$ and $\theta$.

Remark 3.23. In the standard case, it is interesting to take a closer look at the structure of the measure space $(Q, \Sigma, \mu)$. Then $\mathcal{H} \cong L^{2}(M, \mathfrak{S}, \nu)$, and for $L^{\infty}(M, \mathfrak{S}, \nu) \cong C\left(Q_{0}\right)$ we obtain on the compact space $Q:=Q_{0}^{G}$ a measure which is determined by the corresponding cylinder measures:

$$
\int_{Q} f_{g_{1}} \cdots f_{g_{n}} d \mu=\int_{M} M_{f_{1}} P_{g_{1}^{-1} g_{2}} \cdots M_{f_{n-1}} P_{g_{n-1}^{-1} g_{n}} M_{f_{n}} 1 d \nu
$$

for $0 \leq f_{1}, \ldots, f_{n} \in C\left(Q_{0}\right)$ and $g_{1} \prec_{S} \cdots \prec_{S} g_{n}$.

For every $g \in G$, we then have a continuous projection $q_{g}: Q \rightarrow Q_{0}$, so that we obtain a homomorphism $q_{g}^{*}: L^{\infty}(M, \mathfrak{S}, \nu) \cong C\left(Q_{0}\right) \rightarrow C(Q)$ of $C^{*}$-algebras. This leads to a morphism $\mathfrak{S} / J_{\nu} \rightarrow \Sigma / J_{\mu}$ of Boolean $\sigma$-algebras. At this point it is a natural question whether this morphism can be realized by a measurable function $f_{g}: Q \rightarrow M$. In [66, Theorem 1.6] this is answered in the affirmative for $M=\mathbb{R}^{n}$, but this implies the corresponding result for completely metrizable separable spaces, because these spaces can be realized as closed subsets of some $\mathbb{R}^{\mathbb{N}}$. Once we have the measurable maps $f_{g}: Q \rightarrow M$, they combine to a stochastic process $\left(f_{g}\right)_{g \in G}$ with state space $(M, \mathfrak{S})$, so that we obtain an isomorphism of measure spaces $f:(Q, \Sigma, \mu) \rightarrow$ $\left(M^{G}, \mathfrak{S}^{G}, \mu^{\prime}\right)$.

If $(M, \mathfrak{S}, \nu)$ is a polish space, i.e., $M$ carries a topology for which it is completely metrizable and separable and $\mathfrak{S}$ is the $\sigma$-algebra of Borel sets, then [7, Corollary 35.4] directly implies the existence of a Borel measure $\mu^{\prime}$ on the measurable space $\left(M^{G}, \mathfrak{S}^{M}\right)$ with the correct projections onto finite products:

$$
\int_{M^{G}} f_{1}\left(\omega\left(g_{1}\right)\right) \cdots f_{n}\left(\omega\left(g_{n}\right)\right) d \mu^{\prime}(\omega)=\int_{Q} M_{f_{1}} P_{g_{1}^{-1} g_{2}} \cdots M_{f_{n-1}} P_{g_{n-1}^{-1} g_{n}} M_{f_{n}} 1 d \nu
$$

for $0 \leq f_{1}, \ldots, f_{n} \in C\left(Q_{0}\right)$ and $g_{1} \prec_{S} \cdots \prec_{S} g_{n}$. Therefore we obtain a realization of our $(G, S, \tau)$-measure space on $\left(M^{G}, \mathfrak{S}^{G}, \mu^{\prime}\right)$.

Remark 3.24. (a) Consider the case $(G, S, \tau)=\left(\mathbb{R}, \mathbb{R}_{+},-\mathrm{id}_{\mathbb{R}}\right)$ and assume that the operators $P_{t} f$ are obtained from Markov kernels $P_{t}: M \times \mathfrak{S} \rightarrow[0, \infty]$ in the sense that

$$
\left(P_{t} f\right)(x)=\int_{M} P_{t}(x, d y) f(y)
$$

(cf. Appendix B). In view of Remark B.3, the measures $P_{\mathbf{t}}^{\nu}$ can be written as

$$
P_{\mathbf{t}}^{\nu}=\nu P_{\mathbf{t}}, \quad \text { i.e., } \quad P_{\mathbf{t}}^{\nu}(A)=\int_{M} \nu(d x) P_{\mathbf{t}}(x, A)
$$

for the kernel

$$
P_{\mathbf{t}}\left(x_{0}, B\right)=\int_{M^{n}} \chi_{B}\left(x_{1}, \ldots, x_{n}\right) P_{t_{1}}\left(x_{0}, d x_{1}\right) P_{t_{2}-t_{1}}\left(x_{1}, d x_{2}\right) \cdots P_{t_{n}-t_{n-1}}\left(x_{n-1}, d x_{n}\right)
$$

on $M \times \mathfrak{S}^{n}$. In particular, we have for each $x \in M$ a probability measure $P_{x}$ on

$$
\left\{\omega \in M^{\mathbb{R}_{+}}: \omega(0)=x\right\},
$$


defining a kernel $P: M \times \mathfrak{S}^{\mathbb{R}_{+}} \rightarrow[0, \infty]$, such that

$$
P^{\nu}=\nu P, \quad \text { i.e., } \quad P^{\nu}(A)=\int_{M} \nu(d x) P(x, A) .
$$

For any $t \geq 0$, we then have

$$
P_{t}\left(x_{0}, B\right)=\int_{M^{\mathbb{R}_{+}}} \chi_{B}(\omega(t)) P\left(x_{0}, d \omega\right)=P\left(x_{0},\{\omega(t) \in B\}\right),
$$

which leads to

$$
\left(P_{t} f\right)(x)=\int_{M} P_{t}(x, d y) f(y)=\int_{M^{\mathbb{R}_{+}}} P(x, d \omega) f(\omega(t)) .
$$

This is an abstract version of the Feynman-Kac-Nelson formula that expresses the value of $P_{t} f$ in $x \in M$ as an integral over all paths $[0, t] \rightarrow M$ starting in $x$ with respect to the probability measure $P_{x}$.

We also note that, for $t>0$, we have the relation

$$
\int_{M^{\mathbb{R}}} f(\omega(t)) d P^{\nu}(\omega)=\int_{Q} f d \nu \quad \text { for } \quad t \in \mathbb{R},
$$

and, for $t<s$,

$$
\begin{aligned}
\int_{M} \int_{M} f_{1}(x) \nu(d x) f_{2}(y) P_{s-t}(x, d y) & =\int_{M} f_{1}(x)\left(P_{s-t} f_{2}\right)(x) d \nu(x) \\
& =\int_{M^{\mathbb{R}}} f_{1}(\omega(t)) f_{2}(\omega(s)) d P^{\nu}(\omega) .
\end{aligned}
$$

(b) In the special case where $M=G$ is a topological group and $P_{t} f=f * \mu_{t}$, we have

$$
\left(P_{t} f\right)(x)=\int_{G} f(x y) d \mu_{t}(y)=\int_{G} P_{t}(x, d y) f(y) \quad \text { for } \quad P_{t}(x, A)=\mu_{t}\left(x^{-1} A\right) .
$$

Therefore the operators $P_{t}$ are given by Markov kernels. Here, the measurability of the functions

$$
x \mapsto \mu_{t}\left(x^{-1} A\right)=\int_{G} \chi_{A}(x y) d \mu_{t}(y)
$$

follows from the measurability of the function $(x, y) \mapsto \chi_{A}(x y)$.

Let $P(G)$ be the path group $G^{\mathbb{R}}$ and let $P_{*}(G)$ be the subgroup of pinned paths

$$
P_{*}(G)=\{\omega \in P(G): \omega(0)=\mathbf{1}\} .
$$

We have the relations

$$
\int_{P(G)} f_{1}(\omega(0)) f_{2}(\omega(t)) d \mu(\omega)=\int_{G} \int_{G} f_{1}\left(g_{1}\right) f_{2}\left(g_{1} g_{2}\right) d \nu\left(g_{1}\right) d \mu_{t}\left(g_{2}\right) \quad \text { for } \quad t>0
$$

and

$$
\int_{P_{*}(G)} f(\omega(t)) d P^{\nu}(\omega)=\int_{G} f(g) d \mu_{t}(g) .
$$

This leads for $f \in L^{2}(G, \nu)$ and $t \geq 0$ to

$$
\left(P_{t} f\right)(x)=\left(f * \mu_{t}\right)(x)=\int_{G} f(x g) d \mu_{t}(g)=\int_{G^{\mathbb{R}_{+}}} f(x \omega(t)) d P(\omega) .
$$

This is a group version of the Feynman-Kac-Nelson formula (3.6) which expresses $\left(P_{t} f\right)(x)$ as an integral over all paths $\omega:[0, t] \rightarrow G$ with $\omega(0)=x$. 
Remark 3.25. The assumption that $G=S \cup S^{-1}$ is very restrictive (see the discussion in Subsection 3.5). We mainly use it to ensure that the order $\prec_{S}$ on $G$ is total, which is a crucial ingredient in the construction. However, if $S \subseteq G$ is a subsemigroup not satisfying the totality condition, i.e., $S \cup S^{-1} \neq G$, then one may still consider subsets $\mathcal{C} \subseteq G$ on which the order $\prec_{S}$ is total, so-called $\mathcal{C}$-chains. Typical examples of such $\prec_{S}$-chains are curves $\mathcal{C}=\gamma(I), I \subseteq \mathbb{R}$, with $\gamma\left(t_{2}\right) \in \gamma\left(t_{1}\right) S$ for $t_{1}<t_{2}$ in $I$. For any such chain $\mathcal{C}$, one can still construct a stochastic process indexed by $\mathcal{C}$, but we then don't have the symmetries implemented by a $G$-action on the corresponding probability space.

Remark 3.26. The Abel-Klein reconstruction theorem leads in particular to realizations of dilations of the corresponding standard positive semigroup structures $\left(P_{t}\right)_{t \geq 0}$ of the following kind:

(a) Positivity preserving semigroups obtained by integrating a measure preserving action of $G$ on some measure space $(X, \mathfrak{S}, \nu)$ to operators

$$
\left(P_{t} f\right)(x):=\int_{G}(g \cdot f)(x) d \mu_{t}(g)
$$

where the measures $\mu_{t}$ on $G$ are symmetric. If $(X, \mathfrak{S})$ is polish, the corresponding measure space can be realized on the path space $X^{\mathbb{R}}$. Here the requirement that $G$ acts continuously on $L^{2}(X, \mathfrak{S}, \nu)$ implies that $\left(P_{t}\right)_{t \geq 0}$ is strongly continuous on $L^{2}(X, \mathfrak{S}, \nu)$ (see (b) below for a more general argument) and therefore continuous in measure (Remark 3.6(c)).

(b) Semigroups of the type $\left(\Gamma\left(\pi\left(\mu_{t}\right)\right)\right)_{t \geq 0}$, where $(\pi, \mathcal{H})$ is a continuous unitary representation of $G$ on $\mathcal{H}$ and $\Gamma$ is the functor of second quantization (see Section 5.1 for more information about second quantization). Here we use that, for every continuous orthogonal representation $(\pi, \mathcal{H})$ of $G$ and a Borel probability measure $\nu$ on $G$, we can define

$$
\pi(\nu):=\int_{G} \pi(g) d \nu(g)
$$

as the operator representing the hermitian form

$$
q(v, w):=\int_{G}\langle\pi(g) v, w\rangle d \nu(g)
$$

which satisfies $|q(v, w)| \leq\|v\|\|w\|$ for $v, w \in \mathcal{H}$. For any one-parameter semigroup $\left(\mu_{t}\right)_{t \geq 0}$ of symmetric probability measures, we thus obtain a hermitian one-parameter semigroup of contractions $\left(\pi\left(\mu_{t}\right)\right)_{t \geq 0}$. The continuity of this one-parameter semigroup follows from the continuity of the functions

$$
t \mapsto\left\langle\pi\left(\mu_{t}\right) v, w\right\rangle=\int_{G}\langle\pi(g) v, w\rangle d \mu_{t}(g),
$$

which is a consequence of the weak convergence $\mu_{t} \rightarrow \delta_{\mathbf{1}}$ for $t \rightarrow 0$ and the boundedness of the matrix coefficients of $\pi$.

\subsection{Klein's characterization of the Markov case}

The following theorem characterizes the positive semigroup structures arising from Markov path spaces as those for which $\Omega$ is a cyclic vector for $\mathcal{A}$, which is considerably stronger than condition (PS4)(b).

Theorem 3.27 ([41, Theorem 3.1]). Let $\left((Q, \Sigma, \mu), \Sigma_{0}, U, \theta\right)$ be a $(G, S, \tau)$-probability space and let $(\widehat{\mathcal{E}}, \widehat{U}, \mathcal{A}, \Omega)$ be its associated positive semigroup structure. Then $\left((Q, \Sigma, \mu), \Sigma_{0}, U, \theta\right)$ is Markov if and only if $\Omega$ is cyclic for $\mathcal{A}$. 
Proof. The Markov property is equivalent to $q\left(\mathcal{E}_{0}\right)=\widehat{\mathcal{E}}$ (Proposition 3.3). Since $\mathcal{E}_{0}=\mathcal{A} \cdot 1$, this condition is equivalent to $\Omega=q(1)$ being $\mathcal{A}$-cyclic in $\widehat{\mathcal{E}}$.

Remark 3.28 (cf. [41, Remark 3.3]). Suppose that $(\mathcal{H}, P, \mathcal{A}, \Omega)$ is a positive semigroup structure for which $\Omega$ is $\mathcal{A}$-cyclic, so that Proposition 3.7 implies that $\mathcal{H} \cong L^{2}(M)$ for a probability space $M$. Then condition (PS4)(c) follows from the seemingly weaker condition

$$
A, B \in \mathcal{A}_{+}:=\{C \in \mathcal{A}: 0 \leq C\}, \quad s \in S \quad \Rightarrow \quad\left\langle A \Omega, P_{s} B \Omega\right\rangle \geq 0 .
$$

In fact, in this case $\mathcal{A}_{+} \Omega$ is dense in the closed cone $L^{2}\left(Q, \Sigma_{0}, \mu\right)_{+}$of non-negative functions, so that $\left\langle A \Omega, P_{s} B \Omega\right\rangle \geq 0$ for all $A, B \in \mathcal{A}_{+}$implies that the operator $P_{s}$ on $L^{2}\left(Q, \Sigma_{0}, \mu\right)$ is positivity preserving. Then

$$
P_{s_{1}} A_{1} \cdots P_{s_{n}} A_{n} \Omega \geq 0
$$

for $A_{j} \in \mathcal{A}_{+}$and $s_{j} \in S$, and the assertion follows.

Remark 3.29. (a) We consider the Markov case where $\mathcal{A} \Omega$ is dense in $\mathcal{H}$, so that $\mathcal{E}_{0} \cong$ $L^{2}\left(Q, \Sigma_{0}, \mu\right)$ is a $G$-cyclic subspace of $\mathcal{E}$. Then $\varphi(g)=E_{0} U_{g} E_{0}$ satisfies $\varphi(s)=\widehat{U_{s}}=P_{s}$ for $s \in S$. Therefore the $G$-invariant subspace of $\mathcal{E}$ generated by $\mathcal{E}_{0}$ carries a representation which is a unitary dilation of the representation $(P, \mathcal{H})$ of $S$ (cf. Remark 2.8).

(b) The set of finite products $f_{g_{1}} \cdots f_{g_{n}}, g_{j} \in G, f_{j} \in \mathcal{E}_{0} \cong L^{\infty}(M, \mathfrak{S}, \nu) \cap L^{1}(M, \mathfrak{S}, \nu)$ spans a dense subspace of $\mathcal{E}$. Accordingly, the restriction $g_{j} \in S$ leads to a dense subspace of $\mathcal{E}_{+}$. This permits us to give sufficient conditions for $\mathcal{E}_{+}$to be cyclic in $\mathcal{E}$. Since

$$
U_{g} f_{g_{1}} \cdots f_{g_{n}}=f_{g g_{1}} \cdots f_{g g_{n}}
$$

this is the case if, for every finite subset $F \subseteq G$, there exists a $g \in G$ with $g F \subseteq S$, i.e., $g^{-1} \prec_{S} F$. This means that the order $\prec_{S}$ on $G$ is filtered, i.e., for $g_{1}, g_{2} \in G$, there exists a $g_{3} \in G$ with $g_{3} \prec_{S} g_{1}, g_{2}$. This property is easily seen to be equivalent to $G=S^{-1} S$ because $g_{1}^{-1} g_{2} \in S^{-1} S$ is equivalent to the existence of $s_{1}, s_{2} \in S$ with $g_{3}:=g_{2} s_{2}^{-1}=g_{1} s_{1}^{-1} \prec_{S} g_{1}, g_{2}$ (cf. [25]). We conclude that $\mathcal{E}_{+}$is $G$-cyclic in $\mathcal{E}$ if $G=S^{-1} S$. Note that this is in particular the case if $G=S \cup S^{-1}$.

\subsection{Total subsemigroups of Lie groups}

In this section we briefly discuss total subsemigroups. Our standard references are [25, Chapter 6] and [43]. For Lie groups $G$, subsemigroups $S$ with dense interior satisfying $G=S \cup S^{-1}$ are rare. Typical examples are:

(a) $S=\left[0, \infty\left[=\mathbb{R}_{+} \subseteq G=\mathbb{R}\right.\right.$.

(b) $S=\mathbb{R}_{+} \rtimes \mathbb{R}_{+}^{\times} \subseteq G=\mathbb{R} \rtimes \mathbb{R}_{+}^{\times}$(the $a x+b$-group).

(c) A subsemigroup $S \subseteq G=\widetilde{\mathrm{SL}}_{2}(\mathbb{R})$ (the simply connected covering group of $\mathrm{SL}_{2}(\mathbb{R})$ ), whose boundary is a 2-dimensional subgroup isomorphic to the $a x+b$-group. Actually $G$ is the simply connected covering of the projective group $\mathrm{PSL}_{2}(\mathbb{R})$ acting by orientation preserving projective maps on the projective line $\mathbb{P}\left(\mathbb{R}^{2}\right) \cong \mathbb{S}^{1}$. Identifying $\widetilde{\mathbb{S}}^{1}$ with $\mathbb{R}$, we have that

$$
S=\{g \in G: g(0) \geq 0\}=\left\{g \in G: g\left(\mathbb{R}_{+}\right) \subseteq \mathbb{R}_{+}\right\} .
$$

In terms of an Iwasawa decomposition $G=K A N$ with $K \cong \mathbb{R}$ and $K_{+}:=\mathbb{R}_{+}$, we have $S=K_{+} A N=A N K_{+}$and $\mathbb{R} \cong K \cong G / A N$. 
It is easy to see that $S$ is contained in a maximal subsemigroup of $S$ and if $\mathfrak{g} / \operatorname{rad}(\mathfrak{g})$ is a compact Lie algebra, then the classification of maximal subsemigroups $M$ [25, Theorem 6.18] implies that $\partial M$ is a codimension 1 subgroup of $G$. According to the classification of hyperplane subalgebras of finite-dimensional Lie algebras [27, 28], this means that, if $N \subseteq \partial M$ is the largest connected normal subgroup of $G$ contained in $\partial M$, then $M / N \subseteq G / N$ is one of the three types described above. The examples under (a)-(c) are also semigroups whose boundary $\partial S$ is a subgroup, so that $S^{0}$ is well bounded in the sense of $[16,59]$. Such a semigroup, for which $\partial S$ contains no non-trivial connected normal subgroup, is isomorphic to (a), (b) or (c) [16, 59].

From [50] we know that, for the first two types (a) and (b), there are non-trivial reflection positive representations for $(G, S, \tau)$, where $\tau$ is such that $G^{\tau}=\partial S$. On $\mathfrak{s l}_{2}(\mathbb{R})$, the fixed point set of any involutive automorphism is 1-dimensional, hence cannot be a hyperplane. Suppose that, for $G=\widetilde{\mathrm{SL}}_{2}(\mathbb{R}), G^{\tau} \subseteq \partial S$. Then $S$ is invariant under $s \mapsto s^{\sharp}=\tau(s)^{-1}$, but one can show that all involutive continuous representations of $(S, \sharp)$ are trivial. This follows from the Lüscher-Mack theorem [46] and the fact that all involutive contractive representations of the group $(\operatorname{Aff}(\mathbb{R}), \sharp)$ must be trivial on the subgroup $\{0\} \rtimes \mathbb{R}_{+}^{\times}$. This is a consequence of the fact that if, for a selfadjoint operator $A$, all operators $e^{t A}$ are contractions, we have $\operatorname{Spec}(A)=\{0\}$, hence $A=0$.

Example 3.30. $S=\mathbb{R}_{+}^{d} \subseteq G=\mathbb{R}^{d}$ satisfies $G=S \cup S^{-1}$. Since this subsemigroup is invariant under $\mathrm{O}_{d-1}(\mathbb{R})$, we likewise obtain $G_{1}=S_{1} \cup S_{1}^{-1}$ for $G_{1}:=\mathbb{R}^{d} \rtimes \mathrm{O}_{d-1}(\mathbb{R})$ and $S_{1}:=\mathbb{R}_{+}^{d} \rtimes \mathrm{O}_{d-1}(\mathbb{R})$. Note that $\left(G_{1}, \tau_{1}\right)$ with $\tau_{1}(b, A):=(\theta(b), A)$ is a symmetric Lie group with one-dimensional $\mathfrak{q}$.

\subsection{The discrete case}

Our reconstruction theorem applies in particular to $(G, S, \tau)=\left(\mathbb{Z}, \mathbb{N}_{0},-\mathrm{id}_{\mathbb{Z}}\right)$. Here we may start with a Markov operator $P$ on a polish probability space $(M, \mathfrak{S}, \nu)$, so that $P$ is positivity preserving and satisfies $P 1=1$ (cf. Appendix B). If $P$ defines a symmetric operator on $L^{2}(M, \mathfrak{S}, \nu)$, then we obtain a unitary operator $U$ acting by translation on $Q:=M^{\mathbb{Z}}$ such that $E_{0} U E_{0}=P$ holds for the conditional expectation defined by $\Sigma_{0}:=\operatorname{ev}_{0}^{*}(\mathfrak{S}) \subseteq \Sigma$.

An interesting class of examples arises from finite probability spaces $M=\{1, \ldots, n\}$ with the $\sigma$-algebra $2^{M}$. Then a Markov kernel $P$ on $M$ is given by the matrix $P_{i j}:=P(i,\{j\})$ which is stochastic in the sense that $\sum_{j} P_{i j}=1$ for every $i$. If $\mu$ is a measure on $M$, encoded in the row vector $p=\left(p_{j}\right)_{1 \leq j \leq n}$ with $p_{j}:=\mu(\{j\})$, then $\mu P$ is represented by the row vector $p P$. In particular, the invariance condition $\nu P=\nu$ translates into $\sum_{i} p_{i} P_{i j}=p_{j}$ for every $j$. So $P$ acts on measures on $M$ (identified with row vectors) by right multiplication and on functions on $M$ (represented by column vectors) by left matrix multiplication.

If $P$ acts as a symmetric operator on $L^{2}(M, \nu)$, then a Markov process $\left(X_{n}\right)_{n \in \mathbb{Z}}$ can now be obtained from the projections $X_{n}: Q=M^{\mathbb{Z}} \rightarrow M$ and the shift invariant probability measure $\mu$ on $Q$ is determined by

$$
\mu\left(X_{0}=i\right)=p_{i}, \quad \mu\left(X_{1}=i \mid X_{0}=j\right)=P_{i j} .
$$

In this context, we have for integers $t_{1} \leq \cdots \leq t_{n}$ :

$$
\begin{aligned}
\int_{Q} f_{1}\left(\omega\left(t_{1}\right)\right) \cdots f_{m}\left(\omega\left(t_{m}\right)\right) d \mu(\omega) & =\left\langle M_{f_{1}} P^{t_{2}-t_{1}} \cdots M_{f_{m-1}} P^{t_{m}-t_{m-1}} M_{f_{m}} 1,1\right\rangle_{L^{2}(M, \nu)} \\
& =\int_{M} M_{f_{1}} P^{t_{2}-t_{1}} \cdots M_{f_{m-1}} P^{t_{m}-t_{m-1}} f_{m} d \nu(m)
\end{aligned}
$$

(Theorem 3.22). Applying this relation to $\delta$-functions $f_{j}=\delta_{i_{j}}$, we obtain

$$
\mu\left(X_{t_{1}}=i_{1}, \ldots, X_{t_{n}}=i_{n}\right)=p_{i_{1}}\left(P^{t_{2}-t_{1}}\right)_{i_{1} i_{2}}\left(P^{t_{3}-t_{2}}\right)_{i_{2} i_{3}} \cdot\left(P^{t_{n}-t_{n-1}}\right)_{i_{n-1} i_{n}} .
$$


For $n=1$, we obtain in particular

$$
\mu\left(X_{t}=i\right)=p_{i}=\mu\left(X_{0}=i\right) .
$$

For $n=2$ and $t \in \mathbb{N}_{0}$, we obtain

$$
\mu\left(X_{0}=i, X_{t}=j\right)=p_{i}\left(P^{t}\right)_{i j} .
$$

For $t=1$, this leads to

$$
\mu\left(X_{0}=i, X_{1}=j\right)=p_{i} P_{i j} \quad \text { and } \quad \mu\left(X_{1}=j \mid X_{0}=i\right)=P_{i j},
$$

which is (3.7).

\section{Measures on path spaces for topological groups}

In this section we build a bridge between Markov processes and the reconstruction results from the preceding section. To this end, we return to the classical setting, where the symmetric Lie group is $\left(\mathbb{R}, \mathbb{R}_{+},-\mathrm{id}\right)$, but the measure space is the path space $P(Q)=Q^{\mathbb{R}}$ of a polish topological group $Q$.

If $\nu$ is a measure on a locally compact group $Q$ and $\left(\mu_{t}\right)_{t>0}$ is a convolution semigroup of symmetric probability measures on $Q$ satisfying $\nu * \mu_{t}=\nu$ for every $t>0$, then the Klein-Landau reconstruction theorem applies. Since the measure $\nu$ need not be finite, $\nu$ may in particular be the right invariant Haar measure $\mu_{Q}$ on $Q$. The operators $P_{t} f=f * \mu_{t}$ define a positive semigroup structure on $L^{2}(Q, \nu)$, and the reconstruction process leads to a reflection positive unitary representation of $\mathbb{R}$ on $L^{2}\left(P(Q), \mathfrak{B}^{\mathbb{R}}, \nu\right)$ by translation (Theorem 4.9). If $\mu_{t}=\gamma_{t}$ is the gaussian semigroup on $\mathbb{R}^{d}$, this construction leads to an infinite measure on $P\left(\mathbb{R}^{d}\right)$ which realizes in particular the dilation of the heat semigroup as a subrepresentation (cf. [50, 54]).

Here an interesting point is that the map $Q \times P_{*}(Q) \rightarrow P(Q),(q, \omega) \mapsto q \omega$ yields a product decomposition of $P(Q)$ into the subgroup of constant paths and the subgroup $P_{*}(Q)$ of pinned paths. The measures constructed on $P(Q)$ are actually product measures in these coordinates, where $Q$ carries the measure $\nu$ and the measure on $P_{*}(Q)$ is a probability measure determined by the $\mu_{t}$. There is a natural measure preserving $\mathbb{R}$-action on $P_{*}(Q)$ by

$$
\left(V_{t} \omega\right)(s):=\omega(-t)^{-1} \omega(s-t) .
$$

The measure in $P_{*}(Q)$ defines a $Q$-valued stochastic process $\left(X_{t}\right)_{t \in \mathbb{R}}$ with $X_{\mathbf{1}}=\mathbf{1}$ (the constant function). For the special case, where $\mu_{t}=\gamma_{t}$ is the gaussian semigroup on $\mathbb{R}^{d}$, this construction leads to the Wiener measure on $P_{*}\left(\mathbb{R}^{d}\right)$. Presently, we do not know how to obtain a similar factorization if $\left(\mathbb{R}, \mathbb{R}_{+},-\mathrm{id} \mathbb{R}_{\mathbb{R}}\right)$ is replaced by some $(G, S, \tau),{ }^{4}$ so we discuss the one-dimensional case in Subsection 4.1 .

\subsection{One-parameter convolution semigroups of measures on polish groups}

In this subsection $Q$ is a polish topological group. We write $\mathfrak{B}$ for the $\sigma$-algebra of Borel subsets of $Q$. A kernel $K: Q \times \mathfrak{B} \rightarrow[0, \infty]$ on $(Q, \mathfrak{B})$ (cf. Appendix B) is said to be left invariant if

$$
K(g h, g A)=K(h, A) \quad \text { for all } \quad g, h \in Q, A \in \mathfrak{B} .
$$

Then $\mu(A):=K(\mathbf{1}, A)$ is a positive Borel measure on $Q$ such that $K(g, A)=\mu\left(g^{-1} A\right)$. If, conversely, $\mu$ is a $\sigma$-finite Borel measure on $Q$, then $K(g, A):=\mu\left(g^{-1} A\right)$ is a left invariant

\footnotetext{
${ }^{4}$ This is caused by the ambiguities related to the discrepancy between finite subsets $\left\{g_{1}, \ldots, g_{n}\right\}$ of $G$ and increasing tuples $\left(g_{1}, \ldots, g_{n}\right)$ with respect to $\prec_{S}$.
} 
kernel on $Q \times \mathfrak{B}$ because, for every $A \in \mathfrak{B}$, the function $g \mapsto \mu\left(g^{-1} A\right)$ is measurable. In fact, $\widetilde{A}:=\left\{(x, y) \in Q^{2}: x y \in A\right\}$ is a Borel subset of $Q \times Q$, and $\widetilde{A} \cap(\{g\} \times Q)=\{g\} \times g^{-1} A$. We thus obtain a one-to-one correspondence between left invariant Markov kernels $K$ on $Q$ and Borel probability measures $\mu$ on $Q$. Since the product of two kernels corresponds to the convolution product of the corresponding measures, this leads to a one-to-one correspondence between convolution semigroups $\left(\mu_{t}\right)_{t \geq 0}$ of Borel probability measures on $Q$ and left invariant Markov semigroups $\left(P_{t}\right)_{t \geq 0}$ on $(Q, \mathfrak{B})$. We recall from Remark 3.24(b) that the kernel $P_{t}$ acts on functions by right convolution

$$
P_{t} f=f * \mu_{t} \quad \text { for } \quad t \geq 0,
$$

where $f * \mu_{t}(x)=\int_{G} f(x g) d \mu_{t}(g)$.

Consider the path group $P(Q)=Q^{\mathbb{R}}$ and the subgroup

$$
P_{*}(Q):=\{\omega \in P(Q): \omega(0)=\mathbf{1}\} .
$$

Starting with a convolution semigroup $\left(\mu_{t}\right)_{t \geq 0}$, we obtain for the initial distribution $\delta_{\mathbf{1}}$ the Markov process $\left(X_{t}\right)_{t \geq 0}$ with independent stationary increments [7, Corollary 35.4]. The distribution of this process is a probability measure $P^{\mu,+}$ on

$$
P(Q)^{+}=\left\{\omega \in Q^{\mathbb{R}}:(\forall t \leq 0) \omega(t)=\mathbf{1}\right\} \cong Q^{\mathbb{R}_{+}^{\times}} .
$$

For the finite distributions on $Q^{n}$, described by the kernels $P_{\mathbf{t}}, \mathbf{t}=\left(t_{1}, \ldots, t_{n}\right), t_{1}<\cdots<t_{n}$, we obtain with Remark B.3

$$
\begin{aligned}
P_{\mathbf{t}}(B) & =\int_{Q^{n}} \chi_{B}\left(x_{1}, \ldots, x_{n}\right) \mu_{t_{1}}\left(d x_{1}\right) \mu_{t_{2}-t_{1}}\left(x_{1}^{-1} d x_{2}\right) \cdots \mu_{t_{n}-t_{n-1}}\left(x_{n-1}^{-1} d x_{n}\right) \\
& =\int_{Q^{n}} \chi_{B}\left(x_{1}, x_{1} x_{2}, \ldots, x_{1} \cdots x_{n}\right) \mu_{t_{1}}\left(d x_{1}\right) \mu_{t_{2}-t_{1}}\left(d x_{2}\right) \cdots \mu_{t_{n}-t_{n-1}}\left(d x_{n}\right) .
\end{aligned}
$$

This means that

$$
P_{\mathbf{t}}=\left(\psi_{n}\right)_{*}\left(\mu_{t_{1}} \otimes \mu_{t_{2}-t_{1}} \otimes \cdots \otimes \mu_{t_{n}-t_{n-1}}\right)
$$

for the map

$$
\psi_{n}\left(g_{1}, \ldots, g_{n}\right)=\left(g_{1}, g_{1} g_{2}, \ldots, g_{1} \cdots g_{n}\right) .
$$

Remark 4.1. Since the inverse of the map $\psi_{n}$ in (4.2) is given by

$$
\psi_{n}^{-1}\left(h_{1}, \ldots, h_{n}\right)=\left(h_{1}, h_{1}^{-1} h_{2}, \cdots, h_{n-1}^{-1} h_{n}\right),
$$

it follows that the random variables

$$
X_{t_{1}}, X_{t_{1}}^{-1} X_{t_{2}}, \ldots, X_{t_{n-1}}^{-1} X_{t_{n}}
$$

are independent. Since the distribution of $X_{t}^{-1} X_{t+s}$ is $\mu_{s}$ for every $t, s \geq 0$, it follows that $\left(X_{t}\right)_{t \geq 0}$ has independent stationary increments (cf. also [7, Corollary 35.4]).

For a curve $\omega: \mathbb{R} \rightarrow Q$, we define $(\theta \omega)(t):=\omega(-t)$. We write $P(Q)^{ \pm}$for the subgroup consisting of paths whose value is $\mathbf{1}$ on $\mathbb{R}_{-}$, resp., $\mathbb{R}_{+}$and $Q \subseteq P(Q)$ with the subgroup of constant paths. For the following proposition, we recall that the involution on the space $\mathcal{M}(Q)$ of finite real Borel measures on $Q$ is defined by

$$
\int_{Q} f(g) d \mu^{*}(g)=\int_{Q} f\left(g^{-1}\right) d \mu(g) .
$$


Proposition 4.2. Let $P^{\mu,-}:=\theta_{*} P^{\mu,+}$ and $P^{\mu}$ be the image of the measure $P^{\mu,-} \otimes P^{\mu,+}$ on $P_{*}(Q)$ under the bijective product map

$\Phi: \quad P(Q)^{-} \times P(Q)^{+} \rightarrow P_{*}(Q), \quad\left(\omega_{-}, \omega_{+}\right) \mapsto \omega_{-} \cdot \omega_{+}$.

If $\mu_{t}^{*}=\mu_{t}$ holds for every $t \geq 0$, then this measure is invariant under $\theta$ and the one-parameter group of Borel isomorphisms, given by

$$
\left(V_{t} \omega\right)(s):=\omega(-t)^{-1} \omega(s-t), \quad t \in \mathbb{R} .
$$

Proof. The $\theta$-invariance of $P$ follows immediately from its construction and

$$
\theta \Phi\left(\omega_{-}, \omega_{+}\right)=\theta\left(\omega_{-} \cdot \omega_{+}\right)=\theta\left(\omega_{-}\right) \cdot \theta\left(\omega_{+}\right)=\theta\left(\omega_{+}\right) \cdot \theta\left(\omega_{-}\right)=\Phi\left(\theta\left(\omega_{+}\right), \theta\left(\omega_{-}\right)\right) .
$$

We also note that $\theta V_{t} \theta=V_{-t}$ follows from

$$
\left(\theta V_{t} \theta \omega\right)(s)=\left(V_{t} \theta \omega\right)(-s)=(\theta \omega)(-t)^{-1}(\theta \omega)(-s-t)=\omega(t)^{-1} \omega(s+t)=\left(V_{-t} \omega\right)(s) .
$$

Since $P$ is uniquely determined by its images under evaluation in finite tuples $\mathbf{t}=\left(t_{1}, \ldots, t_{n}\right)$, $t_{i}<t_{i+1}$, it suffices to show that the corresponding distributions $P_{\mathbf{t}}$ on $Q^{n}$ satisfy $V_{t} P_{\mathbf{t}}=$ $P_{\left(t_{1}+t, \ldots, t_{n}+t\right)}$. In view of (4.3), we may assume that $t>0$. We may further assume that $t_{k}=0$ for some $k$ and that $t_{m}=-t$ for some $m<k$.

First we note that, for measurable functions $f_{1}, \ldots, f_{k-1}, f_{k+1}, \ldots, f_{n}$ on $Q$, we have

$$
\begin{aligned}
\int_{P_{*}(Q)} & \prod_{j \neq k} f_{j}\left(\omega\left(t_{j}\right)\right) d P^{\mu}(\omega) \\
= & \int_{P(Q)^{+}} \int_{P(Q)^{+}} \prod_{j=1}^{k-1} f_{j}\left(\omega_{-}\left(-t_{j}\right)\right) \prod_{j=k+1}^{n} f_{j}\left(\omega_{+}\left(t_{j}\right)\right) d P^{\mu,-}\left(\omega_{-}\right) d P^{\mu,+}\left(\omega_{+}\right) \\
& \stackrel{(4.1)}{=} \int_{Q^{n-1}} f_{1}\left(g_{k-1} \cdots g_{1}\right) \cdots f_{k-1}\left(g_{k-1}\right) f_{k+1}\left(g_{k+1}\right) \cdots f_{n}\left(g_{k+1} \cdots g_{n}\right) \\
& \times d \mu_{t_{2}-t_{1}}\left(g_{1}\right) \cdots d \mu_{t_{k-1}-t_{k-2}}\left(g_{k-2}\right) d \mu_{-t_{k-1}}\left(g_{k-1}\right) \\
& \times d \mu_{t_{k+1}}\left(g_{k+1}\right) d \mu_{t_{k+2}-t_{k+1}}\left(g_{k+2}\right) \cdots d \mu_{t_{n}-t_{n-1}}\left(g_{n}\right) .
\end{aligned}
$$

For $F(\omega)=\prod_{j=1}^{n} f_{j}\left(\omega\left(t_{j}\right)\right)$, this leads with $t_{m}=-t$ and $t_{k}=0$ to

$$
\begin{aligned}
& \int_{P(Q)}\left(V_{t} F\right)(\omega) d P^{\mu}(\omega)=\int_{P(Q)} F\left(\omega(t)^{-1} \omega(\cdot+t)\right) d P^{\mu}(\omega) \\
& =\int_{P(Q)^{+}} \int_{Q^{m-1}} f_{1}\left(\omega(t)^{-1} g_{m-1} \cdots g_{1}\right) \cdots f_{m-1}\left(\omega(t)^{-1} g_{m-1}\right) f_{m}\left(\omega(t)^{-1}\right) \\
& \quad \times f_{m+1}\left(\omega(t)^{-1} \omega\left(t+t_{m+1}\right) \cdots f_{n}\left(\omega(t)^{-1} \omega\left(t+t_{n}\right)\right)\right) \\
& \quad \times d \mu_{-t_{m-1}-t}\left(g_{m-1}\right) d \mu_{t_{m-1}-t_{m-2}}\left(g_{m-2}\right) \cdots d \mu_{t_{2}-t_{1}}\left(g_{1}\right) d P^{\mu,+}(\omega) \\
& =\int_{Q^{n-1}} \quad f_{1}\left(\left(g_{m} \cdots g_{k-1}\right)^{-1} g_{m-1} \cdots g_{1}\right) \cdots f_{m-1}\left(\left(g_{m} \cdots g_{k-1}\right)^{-1} g_{m-1}\right) f_{m}\left(\left(g_{m} \cdots g_{k-1}\right)^{-1}\right) \\
& \quad \times f_{m+1}\left(\left(g_{m} \cdots g_{k-1}\right)^{-1} g_{m}\right) \cdots f_{k-1}\left(\left(g_{m} \cdots g_{k-1}\right)^{-1} g_{m} \cdots g_{k-2}\right) \\
& \quad \times f_{k+1}\left(g_{k+1}\right) \cdots f_{n}\left(g_{k+1} \cdots g_{n}\right) d \mu_{-t_{m-1}-t}\left(g_{m-1}\right) d \mu_{t_{m-1}-t_{m-2}}\left(g_{m-2}\right) \cdots d \mu_{t_{2}-t_{1}}\left(g_{1}\right) \\
& \quad \times d \mu_{t_{m+1}+t}\left(g_{m}\right) d \mu_{t_{m+2}-t_{m+1}}\left(g_{m+1}\right) \cdots \\
& \quad \times d \mu_{t_{k}-t_{k-1}}\left(g_{k-1}\right) d \mu_{t_{k+1}-t_{k}}\left(g_{k+1}\right) \cdots d \mu_{t_{n}-t_{n-1}}\left(g_{n}\right)
\end{aligned}
$$




$$
\begin{aligned}
& =\int_{Q^{n-1}} \quad f_{1}\left(g_{k-1}^{-1} \cdots g_{m}^{-1} g_{m-1} \cdots g_{1}\right) \cdots f_{m-1}\left(g_{k-1}^{-1} \cdots g_{m}^{-1} g_{m-1}\right) f_{m}\left(g_{k-1}^{-1} \cdots g_{m}^{-1}\right) \\
& \quad \times f_{m+1}\left(g_{k-1}^{-1} \cdots g_{m+1}^{-1}\right) \cdots f_{k-1}\left(g_{k-1}^{-1}\right) f_{k+1}\left(g_{k+1}\right) \cdots f_{n}\left(g_{k+1} \cdots g_{n}\right) \\
& \quad \times d \mu_{t_{2}-t_{1}}\left(g_{1}\right) \cdots d \mu_{t_{m-1}-t_{m-2}}\left(g_{m-2}\right) d \mu_{t_{m}-t_{m-1}}\left(g_{m-1}\right) d \mu_{t_{m+1}-t_{m}}\left(g_{m}\right) \\
& \quad \times d \mu_{t_{m+2}-t_{m+1}}\left(g_{m+1}\right) \cdots d \mu_{t_{k}-t_{k-1}}\left(g_{k-1}\right) d \mu_{t_{k+1}-t_{k}}\left(g_{k+1}\right) \cdots d \mu_{t_{n}-t_{n-1}}\left(g_{n}\right) \\
& \stackrel{(\dagger)}{=} \int_{Q^{n-1}} \quad f_{1}\left(g_{k-1} \cdots g_{1}\right) \cdots f_{m-1}\left(g_{k-1} \cdots g_{m-1}\right) f_{m}\left(g_{k-1} \cdots g_{m}\right) \\
& \quad \times f_{m+1}\left(g_{k-1} \cdots g_{m+1}\right) \cdots f_{k-1}\left(g_{k-1}\right) f_{k+1}\left(g_{k+1}\right) \cdots f_{n}\left(g_{k+1} \cdots g_{n}\right) \\
& \quad \times d \mu_{t_{2}-t_{1}}\left(g_{1}\right) \cdots d \mu_{t_{m}-t_{m-1}}\left(g_{m-1}\right) d \mu_{t_{m+1}-t_{m}}\left(g_{m}\right) d \mu_{t_{m+2}-t_{m+1}}\left(g_{m+1}\right) \cdots \\
& \quad \times d \mu_{t_{k}-t_{k-1}}\left(g_{k-1}\right) d \mu_{t_{k+1}-t_{k}}\left(g_{k+1}\right) \cdots d \mu_{t_{n}-t_{n-1}}\left(g_{n}\right) \\
& \quad \int_{Q^{n-1}}\left(g_{k-1} \cdots g_{1}\right) \cdots f_{k-1}\left(g_{k-1}\right) f_{k+1}\left(g_{k+1}\right) \cdots f_{n}\left(g_{k+1} \cdots g_{n}\right) \\
& \quad \times d \mu_{t_{2}-t_{1}}\left(g_{1}\right) \cdots d \mu_{t_{k}-t_{k-1}}\left(g_{k-1}\right) d \mu_{t_{k+1}-t_{k}}\left(g_{k+1}\right) \cdots d \mu_{t_{n}-t_{n-1}}\left(g_{n}\right) .
\end{aligned}
$$

Here we have used in $(\dagger)$ the symmetry of the measures $\mu_{t}$ to get rid of the inverses. The preceding calculation shows that the transformations $\left(V_{t}\right)_{t \in \mathbb{R}}$ on $P_{*}(Q)$ leave the probability measure $P^{\mu}$ invariant.

Remark 4.3. Note that the transformations $\left(V_{t}\right)_{t \in \mathbb{R}}$ on $P_{*}(Q)$ satisfy

$$
V_{t} X_{s}=X_{t}^{-1} X_{s+t} \quad \text { because } \quad\left(V_{t} X_{s}\right)(\omega)=\left(V_{-t} \omega\right)(s)=\omega(t)^{-1} \omega(s+t) .
$$

On functions on $P_{*}(Q)$, we obtain the action

$$
\left(V_{t} F\right)(\omega)=F\left(\omega(t)^{-1} \omega(\cdot+t)\right)
$$

which is isometric on $L^{2}\left(P_{*}(Q), \mathfrak{B}^{\mathbb{R}^{\times}}, P^{\mu}\right)$.

Example 4.4. (a) (Poisson semigroups) For every $g \in K$, we obtain a convolution semigroup of measures by

$$
\mu_{t}=e^{-t} \sum_{k=0}^{\infty} \frac{t^{k}}{k !} \delta_{g^{k}}=e^{t\left(\delta_{g}-\delta_{0}\right)} \quad \text { for } \quad t \geq 0 .
$$

This semigroup consists of symmetric measures if and only if $\delta_{g}$ is symmetric, i.e., $g=g^{-1}$. In this case $g^{2 n}=\mathbf{1}$ and $g^{2 n+1}=g$ for all $n$, so that

$$
\mu_{t}=\frac{1+e^{-2 t}}{2} \delta_{1}+\frac{1-e^{-2 t}}{2} \delta_{g} .
$$

Note that the limit for $t \rightarrow \infty$ is the measure $\frac{1}{2}\left(\delta_{\mathbf{1}}+\delta_{g}\right)$. This particular example can also be considered as a one-parameter subsemigroup of the semigroup of Markov matrices on $\mathbb{R}^{2}$, resp., Markov operators on the two-element set, where $g$ is the transposition of the two elements (see also Example 3.10).

(b) (Velocity processes) If $K$ is a Lie group and $X \in \mathfrak{g}$, then we obtain a semigroup of measures by

$$
\mu_{t}=\delta_{\exp t X} \quad \text { for } \quad t \geq 0
$$

For these semigroups the condition $\mu_{t}^{*}=\mu_{t}$ for every $t \geq 0$ is satisfied only if $X=0$, i.e., $\mu_{t}=\delta_{\mathbf{1}}$ for every $t$. 
Example 4.5. The class of polish groups is quite large.

(a) Among locally compact groups, the second countable ones carry a complete left invariant metric, turning them in a polish group. This covers in particular all finite-dimensional Lie groups with at most countably many connected components.

(b) Separable Fréchet-Lie groups are also polish, and this class contains in particular all connected Banach-Lie groups whose Lie algebra is separable, gauge groups and groups of diffeomorphisms of compact manifolds.

(c) Another important example of a polish group is the unitary group $\mathrm{U}(\mathcal{H})_{s}$ of a separable Hilbert space $\mathcal{H}$, endowed with the strong operator topology (cf. [47, Proposition II.1] or [62, Theorem II.1, p. 93]).

Remark 4.6. In recent years, many interesting one-parameter semigroups of measures have been studied on infinite-dimensional groups.

(a) On the group $\operatorname{Diff}\left(\mathbb{S}^{1}\right)$ of diffeomorphisms of the circle with respect to the $H^{3 / 2}$-metric, Brownian motion has been constructed by P. Malliavin. A different approach also exhibiting the invariance under rigid rotations is described in [18] (cf. also [21, 22]).

(b) In [10] Brownian motion on compact groups is studied. This is defined to be a stochastic process $\left(X_{t}\right)_{t \geq 0}$ with values in $K$ with $X_{0}=\mathbf{1}$, independent stationary increments and continuous sample paths. In addition, it is assumed to be symmetric, biinvariant and non-degenerate, i.e., $X_{t}$ visits every open subset with positive probability. For the corresponding convolution semigroup $\left(\mu_{t}\right)_{t \geq 0}$ this implies that

$$
\lim _{t \rightarrow 0} t^{-1} \mu_{t}\left(V^{c}\right)=0
$$

for every open 1-neighborhood $V$ in $K$. Conversely, [10, Theorem 1.2] characterizes the convolution semigroups $\left(\mu_{t}\right)_{t \geq 0}$ corresponding to Brownian motions as those which satisfy, in addition to (4.4), that $\mu_{t} \rightarrow \delta_{1}$ weakly on $C(K), \mu_{t}^{*}=\mu_{t}, \mu_{t}$ is conjugation invariant (also called central), and $\operatorname{supp}\left(\mu_{t}\right)=K$ for every $t>0$.

(c) In [17], Driver studies Brownian motion on the infinite-dimensional Banach-Lie group

$$
W(K):=C_{*}([0,1], K)=\{\omega \in C([0,1], K): \omega(0)=\mathbf{1}\},
$$

where $K$ is a connected Lie group with compact Lie algebra, i.e., $K \cong C \times \mathbb{R}^{d}$ for some $d \in \mathbb{N}_{0}$ and a compact Lie group $C$. Here one has to construct a semigroup $\left(\mu_{t}\right)_{t \geq 0}$ of heat kernel measures on the space $W\left(\mathbb{R}^{d}\right)$. On $W\left(\mathbb{R}^{d}\right)$ one has to get hold of the smoothness properties of the heat kernel measures $\mu_{t}$ corresponding to the Wiener measure. The corresponding Hilbert space $H(\mathfrak{g})$ is the space of finite energy paths with values in the Lie algebra $\mathfrak{g}$ and the measures $\mu_{t}$ on $W(K)$ are quasi-invariant under left and right multiplication with elements of the corresponding CameronMartin group $H(K)$ [17, Theorem 7.7]. The unitary representations of $H(K)$ by left, resp., right multiplications has been identified recently with the so-called energy representation of this group [1]. With similar techniques, the existence of heat kernel measures is also obtained for the space of pinned paths with values in a compact Riemannian manifold $M$.

\subsection{Standard path space structures for locally compact groups}

In this section we assume that the group $Q$ is locally compact and that the convolution semigroup $\left(\mu_{t}\right)_{t \geq 0}$ of probability measures on $Q$ is strongly continuous in the sense that $\lim _{t \rightarrow 0} \mu_{t}=\delta_{\mathbf{1}}=\mu_{0}$ weakly on the space $C_{b}(Q)$ of bounded continuous functions on $Q$. We further assume that $\nu$ is a measure on $Q$ satisfying $\nu * \mu_{t}=\nu$ for every $t>0$, and, in addition, that the operators

$$
P_{t} f:=f * \mu_{t}
$$

on $L^{2}(Q, \nu)$ are symmetric. If $\nu$ is a right Haar measure, then the symmetry of the operators $P_{t}$ is equivalent to $\mu_{t}^{*}=\mu_{t}$. 
Remark 4.7. Let $\mu_{Q}$ be a right Haar measure on $Q$ and assume that all the measures $\mu_{t}$ are symmetric. Integrating the right regular representation $\left(\pi^{r}(g) f\right)(x)=f(x g)$ of $Q$ on $L^{2}\left(Q, \mu_{Q}\right)$, we obtain a $*$-representation of the convolution algebra $\mathcal{M}(Q)$ on $L^{2}(Q)$ by

$$
\pi^{r}(\mu):=\int_{Q} \pi^{r}(g) d \mu(g), \quad\left(\pi^{r}(\mu) f\right)(x)=\int_{Q} f(x g) d \mu(g)=(f * \mu)(x) .
$$

Then $P_{t}:=\left(\pi^{r}\left(\mu_{t}\right)\right)_{t \geq 0}$ is a strongly continuous semigroup of hermitian contractions on $L^{2}(Q)$ (here we use $\mu_{t}^{*}=\mu_{t}$ ) which are Markov operators. This is a positive semigroup structure because the continuity in measure (SPS4) follows from the continuity of the action of $Q$ (cf. Remark 3.26). Hence the Klein-Landau reconstruction Theorem 3.22 provides a path space model for the corresponding dilation representation of $\mathbb{R}[50]$.

For $P_{t}$ as above, formula $(3.5)$

$$
\int_{P(Q)} f_{t_{1}} \otimes \cdots \otimes f_{t_{n}} d P_{\mathbf{t}}^{\mu}=\int_{Q} f_{1} P_{t_{2}-t_{1}} f_{2} \cdots P_{t_{n}-t_{n-1}} f_{n} d \nu
$$

in Remark 3.23 specializes for $t_{1} \leq \cdots \leq t_{n}$ and $0 \leq f_{j}$ to

$$
\begin{aligned}
\int_{P(Q)} & f_{1} \otimes \cdots \otimes f_{n} d P_{\mathbf{t}}^{\mu} \\
& =\int_{Q^{n}} f_{1}\left(g_{1}\right) f_{2}\left(g_{1} g_{2}\right) \cdots f_{n}\left(g_{1} \cdots g_{n}\right) d \nu\left(g_{1}\right) d \mu_{t_{2}-t_{1}}\left(g_{2}\right) \cdots d \mu_{t_{n}-t_{n-1}}\left(g_{n}\right) \\
& =\int_{Q^{n}} f_{1}\left(g_{1}\right) f_{2}\left(g_{2}\right) \cdots f_{n}\left(g_{n}\right) d \psi_{*}\left(\nu \otimes \mu_{t_{2}-t_{1}} \otimes \cdots \otimes \mu_{t_{n}-t_{n-1}}\right),
\end{aligned}
$$

where $\psi\left(g_{1}, \ldots, g_{n}\right)=\left(g_{1}, g_{1} g_{2}, \ldots, g_{1} \cdots g_{n}\right)$. We conclude that

$$
P_{\mathbf{t}}^{\mu}=\psi_{*}\left(\nu \otimes \mu_{t_{2}-t_{1}} \otimes \cdots \otimes \mu_{t_{n}-t_{n-1}}\right) .
$$

Lemma 4.8. Let $\nu$ be a measure on $Q$ satisfying $\nu * \mu_{t}=\nu$ for every $t>0$. Then we obtain for $t_{1} \leq \cdots \leq t_{n}$ and $\mathbf{t}:=\left(t_{1}, \ldots, t_{n}\right)$ on $Q^{n}$ a consistent family of measures

$$
P_{\mathbf{t}}^{\mu}:=\left(\psi_{n}\right)_{*}\left(\nu \otimes \mu_{t_{2}-t_{1}} \otimes \cdots \otimes \mu_{t_{n}-t_{n-1}}\right) \text {. }
$$

If $Q$ is a polish group, this leads to a unique measure $P^{\mu}$ on $P(Q)$ with $\left(\mathrm{ev}_{\mathbf{t}}\right)_{*} P^{\mu}=P_{\mathbf{t}}^{\mu}$ for $t_{1}<\ldots<t_{n}$.

Proof. This follows from Remark B.3. For the sake of clarity, we give a direct argument for the consistency of the measures $P_{\mathbf{t}}^{\mu}$. Pick $j \in\{1, \ldots, n\}$ and let $p: Q^{n} \rightarrow Q^{n-1}$ denote the projection omitting the $j$ th component. Then

$$
\left(p \circ \psi_{n}\right)\left(g_{1}, \ldots, g_{n}\right)=\psi_{n-1}\left(g_{1}, \ldots, g_{j-1}, g_{j} g_{j+1}, g_{j+2}, \ldots, g_{n}\right)
$$

implies for $j \geq 2$ that

$$
\begin{aligned}
& p_{*}\left(\psi_{n}\right)_{*}\left(\nu \otimes \mu_{t_{2}-t_{1}} \otimes \cdots \otimes \mu_{t_{n}-t_{n-1}}\right) \\
& \quad=\left(\psi_{n-1}\right)_{*}\left(\nu \otimes \cdots \otimes \mu_{t_{j}-t_{j-1}} * \mu_{t_{j+1}-t_{j}} \otimes \cdots \otimes \mu_{t_{n}-t_{n-1}}\right) \\
& \quad=\left(\psi_{n-1}\right)_{*}\left(\mu_{t_{1}} \otimes \cdots \otimes \mu_{t_{j+1}-t_{j-1}} \otimes \cdots \otimes \mu_{t_{n}-t_{n-1}}\right) .
\end{aligned}
$$

For $j=1$, we obtain

$$
\begin{aligned}
p_{*}\left(\psi_{n}\right)_{*}\left(\nu \otimes \mu_{t_{2}-t_{1}} \otimes \cdots \otimes \mu_{t_{n}-t_{n-1}}\right) & =\left(\psi_{n-1}\right)_{*}\left(\nu * \mu_{t_{2}-t_{1}} \otimes \mu_{t_{3}-t_{2}} \otimes \cdots \otimes \mu_{t_{n}-t_{n-1}}\right) \\
& =\left(\psi_{n-1}\right)_{*}\left(\nu \otimes \mu_{t_{3}-t_{2}} \otimes \cdots \otimes \cdots \otimes \mu_{t_{n}-t_{n-1}}\right) .
\end{aligned}
$$

This implies consistency of the measures $P_{\mathbf{t}}^{\mu}$ on $Q^{n}$. The consistency condition implies the existence of a measure $P^{\mu}$ on $Q^{\mathbb{R}}$ with $\left(\mathrm{ev}_{\mathbf{t}}\right)_{*} \mu=P_{\mathbf{t}}^{\mu}$ for $t_{1} \leq \cdots \leq t_{n}$ (cf. Definition 3.11). 
From the Klein-Landau reconstruction theorem we immediately obtain the following specialization. We refer to [50] for other constructions of this dilation.

Theorem 4.9. Suppose that $Q$ is a second countable locally compact group. Let $\mu$ be the measure on $Q^{\mathbb{R}}$ corresponding to the symmetric convolution semigroup $\left(\mu_{t}\right)_{t \geq 0}$ of probability measures on $Q$ and the measure $\nu$ on $Q$ for which the operators $P_{t} f=f * \mu_{t}$ define a positive semigroup structure on $L^{2}(Q, \nu)$. Then the translation action $\left(U_{t} \omega\right)(s):=\omega(s-t)$ on $P(Q)=Q^{\mathbb{R}}$ is measure preserving and $\mu$ is invariant under $(\theta \omega)(t):=\omega(-t)$. We thus obtain a reflection positive one-parameter group of Markov type on $\mathcal{E}:=L^{2}\left(P(Q), \mathfrak{B}^{\mathbb{R}}, \mu\right)$ with respect to $\mathcal{E}_{+}:=$ $L^{2}\left(P(Q), \mathfrak{B}^{\mathbb{R}_{+}}, \mu\right)$, for which $\mathcal{E}_{0}:=\operatorname{ev}_{0}^{*}\left(L^{2}(Q, \nu)\right) \cong L^{2}(Q, \nu)$ and $\widehat{\mathcal{E}} \cong L^{2}(Q, \nu)$ with $q(F)=E_{0} F$ for $F \in \mathcal{E}_{+}$. We further have

$$
E_{0} U_{t} E_{0}=P_{t} \quad \text { holds for } \quad P_{t} f=f * \mu_{t},
$$

so that the $U$-cyclic subrepresentation generated by $\mathcal{E}_{0}$ is a unitary dilation of the hermitian one-parameter semigroup $\left(P_{t}\right)_{t \geq 0}$ on $L^{2}(Q, \nu)$.

Example 4.10. (a) For $Q=\mathbb{R}^{d}$, the heat semigroup is given on $L^{2}\left(\mathbb{R}^{d}\right)$ by

$$
e^{t \Delta} f=f * \gamma_{t} \quad \text { where } \quad d \gamma_{t}(x)=\frac{1}{(2 \pi t)^{d / 2}} e^{-\frac{1}{2} \frac{\|x\|^{2}}{t}} d x
$$

We call the corresponding measure on $Q^{\mathbb{R}}$ the Lebesgue-Wiener measure (cf. Theorem 4.9).

(b) If $Q$ is a finite-dimensional Lie group and $X_{1}, \ldots, X_{n}$ is a basis of the Lie algebra, then we

obtain a left invariant Laplacian by $\Delta:=\sum_{j=1}^{n} L_{X_{j}}^{2}$, where $L_{X_{j}}$ denotes the right invariant vector field with $L_{X_{j}}(\mathbf{1})=X_{j}$. Then there also exists a semigroup $\left(\mu_{t}\right)_{t \geq 0}$ of probability measures on $Q$ such that $[53$, Section 8]

$$
e^{t \Delta} f=f * \mu_{t} \quad \text { for } \quad t \geq 0 .
$$

Accordingly, we obtain a Haar-Wiener measure on the path space $Q^{\mathbb{R}}$.

\section{Gaussian $(G, S, \tau)$-probability spaces}

In this section we discuss the second quantization functor and its connection to gaussian $(G, S, \tau)$ probability spaces. We then discuss equivalence of gaussian measures for reproducing kernel Hilbert spaces. The main results of this section are contained in Subsection 5.4, where we discuss gaussian measures on the space of distributions on a Lie group. Here the distribution vectors of unitary representations play an important role.

\subsection{Second quantization and gaussian processes}

Definition 5.1. Let $\mathcal{H}$ be a real Hilbert space. A gaussian random process indexed by $\mathcal{H}$ is a random process $(\varphi(v))_{v \in \mathcal{H}}$ on a probability space $(Q, \Sigma, \mu)$ such that

(GP1) $\{\varphi(v): v \in \mathcal{H}\}$ is full, i.e., these random variables generate the $\sigma$-algebra $\Sigma$ modulo zero sets.

(GP2) Each $\varphi(v)$ is a gaussian random variable of mean zero.

(GP3) $\langle\varphi(v), \varphi(w)\rangle=\langle v, w\rangle$ is the inner product on $\mathcal{H}$. 
According to [64, Theorems I.6, I.9], gaussian random processes indexed by $\mathcal{H}$ exist and are unique up to isomorphisms of probability spaces. This means that, if $(Q, \Sigma, \mu)$ is the corresponding probability space, then the algebra $L^{\infty}(Q, \mu)$ with its state given by $\mu$ is uniquely determined by $\mathcal{H}$ (cf. [64, Section 1.1]). Its projections correspond to $\Sigma / J_{\mu}$, where $J_{\mu}$ is the ideal of zero $\operatorname{sets}^{5}$.

Theorem 5.2. Let $V$ be a real vector space, $V^{*}$ be its algebraic dual and $\mathfrak{B}^{*}$ be the smallest $\sigma$ algebra for which all evaluation functions $V^{*} \rightarrow V, \alpha \mapsto \alpha(v)$, are measurable. Then a function $\chi: V \rightarrow \mathbb{C}$ is the Fourier transform $\chi=\widehat{\mu}$ of a measure $\mu$ on $\left(V^{*}, \mathfrak{B}^{*}\right)$ if and only if $\chi$ is positive definite and continuous on every finite-dimensional subspace. In this case $\mu$ is uniquely determined.

Proof. Since the Fourier transform $\widehat{\mu}$ is positive definite and sequentially continuous, it is in particular continuous on every finite-dimensional subspace of $V$. The converse follows from [68, Theorem 16.2].

Example 5.3. Theorem 5.2 implies that the gaussian process indexed by $\mathcal{H}$ may be realized by the probability measure $\gamma_{\mathcal{H}}$ on $\left(\mathcal{H}^{a}, \mathfrak{B}^{*}\right)$ (the algebraic dual) whose Fourier transform is

$$
\widehat{\gamma}_{\mathcal{H}}(v)=e^{-\frac{\|v\|^{2}}{2}} .
$$

Here $\varphi(v)(\alpha)=\alpha(v)$, and

$$
\int_{\mathcal{H}^{a}} e^{i \alpha(v)} d \gamma_{\mathcal{H}}(\alpha)=e^{-\frac{\|v\|^{2}}{2}} \quad \text { implies } \quad \int_{\mathcal{H}^{a}} \alpha(v)^{2} d \gamma_{\mathcal{H}}(\alpha)=\|v\|^{2} .
$$

This leads to

$$
\left\langle e^{i \varphi(v)}, e^{i \varphi(w)}\right\rangle=e^{-\frac{\|v-w\|^{2}}{2}}=e^{-\frac{\|v\|^{2}}{2}} e^{-\frac{\|w\|^{2}}{2}} e^{\langle v, w\rangle},
$$

so that $K_{v}:=e^{i \varphi(v)} e^{\frac{\|v\|^{2}}{2}}$ satisfies

$$
\left\langle K_{v}, K_{w}\right\rangle=e^{\langle v, w\rangle} .
$$

Example 5.4. If $\mathcal{H}$ is finite-dimensional, so that $\mathcal{H} \cong \mathbb{R}^{d}$ for some $d \in \mathbb{N}_{0}$, then $\mathcal{H}^{a} \cong \mathbb{R}^{d}$ and $d \gamma_{\mathcal{H}}(x)=\frac{1}{(2 \pi)^{d / 2}} e^{-\frac{\|x\|^{2}}{2}} d x$ is a gaussian measure.

We now introduce the second quantization. Conceptually the easiest way to define second quantization as a functor is to associate to a (real or complex) Hilbert space $\mathcal{H}$ its Fock space

$$
\mathcal{F}(\mathcal{H}):=\widehat{\bigoplus}_{n \in \mathbb{N}_{0}} S^{n}(\mathcal{H})
$$

where $S^{n}(\mathcal{H}):=\left(\mathcal{H}^{\otimes n}\right)^{S_{n}}$ is the closed subspace of $S_{n}$-invariant vectors in the $n$-fold tensor power of $\mathcal{H}$. For $v_{1}, \ldots, v_{n} \in \mathcal{H}$, we define the symmetric product by

$$
v_{1} \vee \cdots \vee v_{n}:=P_{+}\left(v_{1} \otimes \cdots \otimes v_{n}\right),
$$

\footnotetext{
${ }^{5} \mathrm{~A}$ short proof for the uniqueness can be derived from reproducing kernel techniques. If $\varphi_{j}: \mathcal{H} \rightarrow L^{2}\left(Q_{j}, \mu_{j}\right)$, $j=1,2$, are two realizations, then the corresponding covariance kernels on $\mathcal{H}$ coincide, so that there exists a unique unitary operator $\Phi: L^{2}\left(Q_{1}, \mu_{1}\right) \rightarrow L^{2}\left(Q_{2}, \mu_{2}\right)$ such that $\Phi \circ \varphi_{1}=\varphi_{2}$. Accordingly, $\Phi \circ \mathcal{A}_{1} \circ \Phi^{-1}=\mathcal{A}_{2}$ for the von Neumann algebras $\mathcal{A}_{j}:=\left\{e^{i \varphi_{j}(v)}: v \in \mathcal{H}\right\}^{\prime \prime} \cong L^{\infty}\left(Q_{j}, \mu_{j}\right)$.
} 
where $P_{+}: \mathcal{H}^{\otimes n} \rightarrow S^{n}(\mathcal{H})$ is the orthogonal projection. The inner products of such elements are given by

$$
\begin{aligned}
\left\langle v_{1} \vee \cdots \vee v_{n}, w_{1} \vee \cdots \vee w_{n}\right\rangle & =\left\langle v_{1} \vee \cdots \vee v_{n}, w_{1} \otimes \cdots \otimes w_{n}\right\rangle \\
& =\frac{1}{n !} \sum_{\sigma \in S_{n}}\left\langle v_{\sigma(1)}, w_{1}\right\rangle \cdots\left\langle v_{\sigma(n)}, w_{m}\right\rangle
\end{aligned}
$$

In particular, with $v^{n}=\underbrace{v \vee \cdots \vee v}_{n \text {-times }}$, we have

$$
\left\langle v^{n}, w^{n}\right\rangle=\langle v, w\rangle^{n} \quad \text { and } \quad\left\|v^{n}\right\|=\|v\|^{n} .
$$

Clearly, every contraction $A: \mathcal{H} \rightarrow \mathcal{K}$ defines a contraction $\Gamma(A): \mathcal{F}(\mathcal{H}) \rightarrow \mathcal{F}(\mathcal{K})$ by

$$
\Gamma(A)\left(v_{1} \vee \cdots \vee v_{n}\right):=A v_{1} \vee \cdots \vee A v_{n}
$$

and it is clear that $\Gamma(A B)=\Gamma(A) \Gamma(B)$ and $\Gamma\left(A^{*}\right)=\Gamma(A)^{*}$. In particular, we obtain a representation of the involutive semigroup of contractions on $\mathcal{H}$ in the Fock space $\mathcal{F}(\mathcal{H})$ and $\mathcal{F}$ defines an endofunctor from the category of Hilbert spaces whose morphism are contractions into itself. The problem with this approach is that it completely ignores positivity issues.

If $\mathcal{H}$ is a Hilbert space, then any $v \in \mathcal{H}$ defines a function $\langle v, \cdot\rangle, u \mapsto\langle v, u\rangle$, which is linear if $\mathcal{H}$ is real and antilinear if $\mathcal{H}$ is complex. For

$$
\operatorname{Exp}(v):=\sum_{n=0}^{\infty} \frac{1}{\sqrt{n !}} v^{n}
$$

we now derive from $(5.1)$ that $\operatorname{Exp}(v) \in \mathcal{F}(\mathcal{H})$ and

$$
\langle\operatorname{Exp}(v), \operatorname{Exp}(w)\rangle=\sum_{n=0}^{\infty} \frac{1}{n !}\langle v, w\rangle^{n}=e^{\langle v, w\rangle} .
$$

This leads to an embedding

$$
\Phi: \mathcal{F}(\mathcal{H}) \rightarrow \mathbb{C}^{\mathcal{H}}, \quad \Phi(\xi)(v):=\langle\xi, \operatorname{Exp}(v)\rangle
$$

where

$$
\Phi\left(v_{1} \vee \cdots \vee v_{n}\right)(v)=\frac{1}{\sqrt{n !}}\left\langle v_{1} \vee \cdots \vee v_{n}, v^{n}\right\rangle=\frac{1}{\sqrt{n !}} \prod_{j=1}^{n}\left\langle v_{j}, v\right\rangle
$$

The image of $\Phi$ is the reproducing kernel space $\mathcal{F}_{\mathrm{RK}}(\mathcal{H})$ with kernel

$$
K(v, w)=\langle\operatorname{Exp}(w), \operatorname{Exp}(v)\rangle=e^{\langle w, v\rangle} .
$$

We may thus identify $\mathcal{F}(\mathcal{H})$ with the reproducing kernel Hilbert space $\mathcal{F}_{\mathrm{RK}}(\mathcal{H})$.

For a contraction $A: \mathcal{H} \rightarrow \mathcal{K}$, we have

$$
\Phi\left(A v_{1} \vee \cdots \vee A v_{n}\right)(v)=\frac{1}{\sqrt{n !}}\left\langle A v_{1} \vee \cdots \vee A v_{n}, v^{n}\right\rangle=\frac{1}{\sqrt{n !}}\left\langle v_{1} \vee \cdots \vee v_{n},\left(A^{*} v\right)^{n}\right\rangle
$$

so that the operator $\Gamma(A)$ acts on the reproducing kernel space $\mathcal{F}_{\mathrm{RK}}(\mathcal{H}) \rightarrow \mathcal{F}_{\mathrm{RK}}(\mathcal{K})$ simply by

$$
(\Gamma(A) F)(v):=F\left(A^{*} v\right), \quad\|A\| \leq 1, \quad F \in \mathcal{H}_{K} \subseteq \mathbb{C}^{\mathcal{H}}, \quad v \in \mathcal{K} .
$$




\subsection{Application to reflection positive representations}

Typical examples of gaussian $(G, S, \tau)$-probability spaces arise as follows. Let $\mathcal{H}$ be a real Hilbert space and $\left(Q, \Sigma, \gamma_{\mathcal{H}}\right)$ be a realization of the gaussian random process $(\varphi(v))_{v \in \mathcal{H}}$ indexed by $\mathcal{H}$ (Definition 5.1). Using the realization from Example 5.3, where $Q=\mathcal{H}^{a}$ is the algebraic dual space, we obtain an action of the orthogonal group $\mathrm{O}(\mathcal{H})$ on $(Q, \Sigma, \mu)$ by measure preserving automorphisms.

For the following proposition, we recall the concept of a reflection positive representation of $(G, S, \tau)$ from Definition 2.6.

Lemma 5.5. If $\pi: S \rightarrow B(\mathcal{H})$ be is a continuous $*$-representation of the topological involutive semigroup $(S, \sharp)$ by contractions, then we obtain on $\Gamma(\mathcal{H}) \cong L^{2}\left(\mathcal{H}^{a}, \gamma_{\mathcal{H}}\right)$ by $P_{s}:=\Gamma(\pi(s))$ a standard positive semigroup structure on the probability space $\left(\mathcal{H}^{a}, \gamma_{\mathcal{H}}\right)$.

Proof. Here we use that

$$
\varepsilon^{2} \gamma_{\mathcal{H}}\left(\left\{\left|P_{s} f-P_{s_{0}} f\right| \geq \varepsilon\right\}\right) \leq \int_{\mathcal{H}^{a}}\left|P_{s} f-P_{s_{0}} f\right|^{2} d \gamma_{\mathcal{H}} \rightarrow 0
$$

for $s \rightarrow s_{0}$ follows from the continuity of the representation $\Gamma \circ \pi$ of $S$ on $\Gamma(\mathcal{H})$ (cf. Lemma A.1).

Proposition 5.6. Let $\left(U, \mathcal{E}, \mathcal{E}_{+}, \theta\right)$ be a reflection positive orthogonal representation of $(G, S, \tau)$ for which $\mathcal{E}_{0}$ is $U$-cyclic and $\mathcal{E}_{+}$is generated by $\left(U_{s} \mathcal{E}_{0}\right)_{s \in S}$. Then second quantization leads to $a(G, S, \tau)$-probability space $\left(\left(Q_{\mathcal{H}}, \Sigma, \gamma_{\mathcal{H}}\right), \Sigma_{0}, \Gamma(U), \Gamma(\theta)\right)$, where $\Sigma_{0} \subseteq \Sigma$ is the smallest $\sigma$-algebra for which the functions $(\varphi(v))_{v \in \mathcal{E}_{0}}$ are measurable.

Proof. (GP1-4) Clearly, every $\Gamma\left(U_{g}\right)$ and $\Gamma(\theta)$ are automorphisms of the algebra $L^{\infty}\left(Q_{\mathcal{H}}, \Sigma, \gamma_{\mathcal{H}}\right)$ satisfying

$$
\Gamma(\theta) \Gamma\left(U_{g}\right) \Gamma(\theta)=\Gamma\left(\theta U_{g} \theta\right)=\Gamma\left(U_{\tau(g)}\right) \quad \text { and } \quad \theta E_{0}=E_{0} .
$$

The continuity of the unitary representation $\left(\Gamma\left(U_{g}\right)\right)_{g \in G}$ on $L^{2}\left(Q_{\mathcal{H}}, \gamma_{\mathcal{H}}\right)$ implies the continuity in measure of the $G$-action on $L^{\infty}\left(Q_{\mathcal{H}}, \gamma_{\mathcal{H}}\right)$ (Lemma 5.5).

(GP5) Our definition of $\Sigma_{0}$ implies that $\Sigma_{+}$is the smallest $\sigma$-algebra for which the functions $(\varphi(v))_{v \in \mathcal{E}_{+}}$are measurable and since $\mathcal{E}_{0}$ is $U$-cyclic in $\mathcal{E}$, (GP5) is also satisfied.

Reflection positivity of the representation $\Gamma(U)$ of $(G, S, \tau)$ follows from [50, Remark 3.8] and $\Gamma\left(\mathcal{E}_{+}\right)=L^{2}\left(Q_{\mathcal{H}}, \Sigma_{+}, \gamma_{\mathcal{H}}\right)$.

\subsection{Equivalence of gaussians measures for reproducing kernel Hilbert spaces}

Let $X$ be a set and $E=\mathbb{C}[X]$ the free complex vector spaces over $X$. Then positive definite kernels $K$ on $X$ are in one-to-one correspondence with positive semidefinite hermitian forms on $E$. Any such kernel defines a Hilbert subspace $\mathcal{H}_{K} \subseteq E^{*}$ with continuous point evaluations.

More generally, we may consider for a real locally convex space $E$ continuous bilinear hermitian kernels $K: E \times E \rightarrow \mathbb{C}$ and the corresponding subspaces of the topological dual space $E^{\prime}$ [61]. Such a kernel is positive definite if and only if the canonical sesquilinear extension to the complexification $E_{\mathbb{C}}$ is a positive semidefinite hermitian form. Suppose that $E$ is nuclear. Then, for any such $K$, the function

$$
\varphi_{K}(v):=e^{-\frac{1}{2} K(v, v)}
$$

on $E$ is continuous and positive definite, hence is the Fourier transform of a uniquely determined gaussian measure $\gamma_{K}$ on $E^{\prime}$. We want to express conditions on pairs of kernels $K$ and $Q$ which characterize the equivalence of the measures $\gamma_{K}$ and $\gamma_{Q}$ on $E^{\prime}$. 
According to [38], two gaussian measures are either mutually singular of equivalent. The following theorem is a reformulation of [68, Theorem 10.1] (cf. also [37, Theorem 4.1/4.2] or [11, Corollary 6.4.11]).

Theorem 5.7. Let $E$ be a nuclear real locally convex space. For two continuous positive semidef inite hermitian forms $K$ and $Q$ on $E$, the corresponding gaussian measures $\gamma_{K}$ and $\gamma_{Q}$ on $E^{\prime}$ are equivalent if and only if $\mathcal{H}_{K}=\mathcal{H}_{Q}$ and there exists an operator $T \in \operatorname{GL}\left(\mathcal{H}_{K}\right)$ for which $T T^{*}-\mathbf{1}$ is Hilbert-Schmidt and

$$
Q(x, y)=\left\langle T K_{y}, T K_{x}\right\rangle \quad \text { for } \quad x, y \in E .
$$

Remark 5.8. (a) That the gaussian measure $\gamma_{K}$ on $E^{\prime}$ determines the Hilbert subspace $\mathcal{H}_{K} \subseteq E^{\prime}$ follows from [68, Theorem 9.1] which asserts that $\mathcal{H}_{K}$ consists precisely of those linear functionals $\alpha \in E^{\prime}$ for which $\gamma_{K}$ is quasi-invariant under the translation $\tau_{\alpha}(\beta):=\alpha+\beta$. As a consequence, the equivalence of $\gamma_{K}$ and $\gamma_{Q}$ implies $\mathcal{H}_{K}=\mathcal{H}_{Q}$.

(b) Recall from [48, Theorem I.2.8] that $\mathcal{H}_{K}=\mathcal{H}_{Q}$ is equivalent to the existence of positive constants $c_{1}, c_{2}>0$ such that the kernels

$$
K-c_{1} Q \quad \text { and } \quad c_{2} Q-K
$$

are positive definite. If $K$ and $Q$ are real-valued, this is equivalent to

$$
c_{1} Q(x, x) \leq K(x, x) \leq c_{2} Q(x, x) \quad \text { for } \quad x \in E .
$$

This in turn is equivalent to the existence of a bounded invertible positive operator $A \in B\left(\mathcal{H}_{K}\right)$ such that

$$
Q(x, y)=K^{A}(x, y):=\left\langle A K_{y}, K_{x}\right\rangle \quad \text { for } \quad x, y \in E .
$$

In view of [48, Corollary I.2.6], we have $A=T T^{*}$, where $T: \mathcal{H}_{Q} \rightarrow \mathcal{H}_{K}, f \mapsto f$ is the identity. This implies that

$$
T^{*}\left(K_{x}\right)=Q_{x} \quad \text { for } \quad x \in E .
$$

In particular, we have

$$
Q(x, y)=\left\langle Q_{x}, Q_{y}\right\rangle_{\mathcal{H}_{Q}}=\left\langle T T^{*} K_{x}, K_{y}\right\rangle_{\mathcal{H}_{K}}
$$

We conclude that, for $f \in \mathcal{H}_{Q}=\mathcal{H}_{K}$, we have

$$
\|f\|_{\mathcal{H}_{K}}^{2}=\|T f\|_{\mathcal{H}_{K}}^{2}=\left\langle T^{*} T f, f\right\rangle_{\mathcal{H}_{Q}}
$$

Therefore the equivalence of the corresponding gaussian measure is equivalent to $T^{*} T-\mathbf{1}$ being Hilbert-Schmidt.

Remark 5.9. Let $E$ be a real vector space and endow it with the finest locally convex topology for which all seminorms on $E$ are continuous. Then $E$ is nuclear if and only if $E$ is of at most countable dimension [65, Proposition 50.1, Theorem 51.2]. In any case, its topological dual space is $E^{\prime}=E^{*}$ because every linear functional on $E$ is continuous.

Regardless of the nuclearity of $E$, every positive definite function $\varphi: E \rightarrow \mathbb{C}$ which is continuous on all finite-dimensional subspace is the Fourier transform of a $\mathfrak{B}^{*}$-measure on $E^{*}$ (Theorem 5.2). This applies in particular to all functions of the form $\varphi(v):=e^{-\frac{1}{2} K(v, v)}$, where $K: E \times E \rightarrow \mathbb{R}$ is a positive semidefinite symmetric bilinear form on $E$. 


\subsection{Gaussian measures on distributions on Lie groups}

If $G$ is a Lie group, then $\mathcal{D}(G):=C_{c}^{\infty}(G)$ is an involutive algebra with respect to the convolution product and the involution $\varphi^{*}(g):=\overline{\varphi\left(g^{-1}\right)} \Delta_{G}\left(g^{-1}\right)$, where $\Delta_{G}$ is the modular function satisfying

$$
\Delta_{G}(y) \int_{G} f(x y) d \mu_{G}(x)=\int_{G} f(x) d \mu_{G}(x) \quad \text { for } \quad f \in C_{c}(G), y \in G .
$$

Accordingly, we call a distribution $D \in \mathcal{D}^{\prime}(G)$ (the space of antilinear continuous functionals on $\mathcal{D}(G))$ positive definite, if it is a positive functional on this algebra, i.e.,

$$
D\left(\varphi^{*} * \varphi\right) \geq 0 \text { for } \varphi \in \mathcal{D}(G) \text {. }
$$

Since $\mathcal{D}(G)$ is nuclear, every positive definite distribution $D \in \mathcal{D}^{\prime}(G)$ determines a gaussian measure $\gamma_{D}$ on $\mathcal{D}^{\prime}(G)$.

For a unitary representation $(\pi, \mathcal{H})$ of $G$ we write $\mathcal{H}^{-\infty}$ for the space of continuous antilinear functionals on $\mathcal{H}^{\infty}$, the space of distribution vectors, and note that we have a natural linear embedding $\mathcal{H} \hookrightarrow \mathcal{H}^{-\infty}, v \mapsto\langle v, \cdot\rangle$. Accordingly, we also write $\langle\alpha, v\rangle=\overline{\langle v, \alpha\rangle}$ for $\alpha(v), \alpha \in \mathcal{H}^{-\infty}$ and $v \in \mathcal{H}^{\infty}$. The group $G$ acts naturally on $\mathcal{H}^{-\infty}$ by

$$
\left(\pi^{-\infty}(g) \alpha\right)(v):=\alpha\left(\pi(g)^{-1} v\right)
$$

so that we obtain a $G$-equivariant chain of continuous inclusions

$$
\mathcal{H}^{\infty} \subseteq \mathcal{H} \subseteq \mathcal{H}^{-\infty}
$$

(cf. [14, Section 8.2]). It is $\mathcal{D}(G)$-equivariant, if we define the representation of $\mathcal{D}(G)$ on $\mathcal{H}^{-\infty}$ by

$$
\left(\pi^{-\infty}(\varphi) \alpha\right)(v):=\int_{G} \varphi(g) \alpha\left(\pi(g)^{-1} v\right) d \mu_{G}(g)=\alpha\left(\pi\left(\varphi^{*}\right) v\right)
$$

Proposition 5.10 ([49, Proposition 2.8]). Let $D \in \mathcal{D}^{\prime}(G)$ be a positive definite distribution on the Lie group $G$ and $\mathcal{H}_{D}$ be the corresponding reproducing kernel Hilbert space with kernel $K(\varphi, \psi):=D\left(\psi^{*} * \varphi\right)$ obtained by completing $\mathcal{D}(G) * D$ with respect to the scalar product

$$
\langle\psi * D, \varphi * D\rangle=D\left(\psi^{*} * \varphi\right) .
$$

Then the following assertions hold:

(i) $\mathcal{H}_{D} \subseteq \mathcal{D}^{\prime}(G)$ and the inclusion $\gamma_{D}: \mathcal{H}_{D} \rightarrow \mathcal{D}^{\prime}(G)$ is continuous.

(ii) We have a unitary representation $\left(\pi_{D}, \mathcal{H}_{D}\right)$ of $G$ by

$$
\pi_{D}(g) E=g_{*} E, \quad \text { where }\left(g_{*} E\right)(\varphi):=E\left(\varphi \circ \lambda_{g}\right)
$$

and the integrated representation of $\mathcal{D}(G)$ on $\mathcal{H}_{D}$ is given by $\pi_{D}(\varphi) E=\varphi * E$.

(iii) There exists a unique distribution vector $\alpha_{D} \in \mathcal{H}_{D}^{-\infty}$ with $\alpha_{D}(\varphi * D)=D(\varphi)$ and

$$
\pi^{-\infty}(\varphi) \alpha_{D}=\varphi * D \quad \text { for } \quad \varphi \in \mathcal{D}(G)
$$

(iv) $\gamma_{D}$ extends to a $\mathcal{D}(G)$-equivariant injection $\mathcal{H}_{D}^{-\infty} \hookrightarrow \mathcal{D}^{\prime}(G)$ mapping $\alpha_{D}$ to $D$. 
Remark 5.11. We consider a reflection positive distribution vector $\alpha \in \mathcal{H}^{-\infty}$ for a unitary representation $(\pi, \mathcal{H})$ of $G$. This leads to an embedding

$$
\eta_{\alpha}: \mathcal{H} \rightarrow \mathcal{D}^{\prime}(G), \quad \eta_{\alpha}(v)(\varphi):=\left\langle v, \pi^{-\infty}(\varphi) \alpha\right\rangle=\left\langle\pi\left(\varphi^{*}\right) v, \alpha\right\rangle .
$$

which is injective if and only if $\alpha$ is cyclic. This establishes a one-to-one correspondence between distribution vectors and $G$-equivariant continuous linear maps $\mathcal{H} \rightarrow \mathcal{D}^{\prime}(G)$ (Proposition 5.10). Actually we obtain an equivariant embedding

$$
\mathcal{H}^{-\infty} \hookrightarrow \mathcal{D}^{\prime}(G)
$$

by dualizing the linear map $\mathcal{D}(G) \rightarrow \mathcal{H}, \varphi \mapsto \pi^{-\infty}(\varphi) \alpha$. This in turn leads to the positive definite function

$$
S(\varphi):=e^{-\frac{1}{2}\left\|\pi^{-\infty}(\varphi) \alpha\right\|^{2}}=e^{-\frac{1}{2} D\left(\varphi^{*} * \varphi\right)} \quad \text { for } \quad D(\varphi):=\alpha\left(\pi^{-\infty}(\varphi) \alpha\right), \quad \varphi \in \mathcal{D}(G) .
$$

We thus obtain a $G$-invariant gaussian probability measure $\gamma_{D}$ on $\mathcal{D}^{\prime}(G)$ by Minlos' theorem.

Lemma 5.12. If $\alpha \in \mathcal{H}^{-\infty}$ is cyclic and $(\rho, V)$ is a finite-dimensional irreducible representation of $G$, then the multiplicity of $\rho$ in $\mathcal{H}$ is bounded by $\operatorname{dim} V$. In particular, the $V$-isotypic subspace of $\mathcal{H}$ is finite-dimensional and $\operatorname{dim} \mathcal{H}^{G} \leq 1$.

Proof. Let $n \in \mathbb{N}$ and assume that $V^{n}$ is a subrepresentation of $\mathcal{H}$. Then $V^{n}$ is finite-dimensional and generated by a distribution vector which actually must be an element $\left(v_{1}, \ldots, v_{n}\right)$ $\in V^{n}$. Suppose that $\lambda_{1}, \ldots, \lambda_{n} \in \mathbb{C}$ satisfy $\sum_{j} \lambda_{j} v_{j}=0$. Then $\sum_{j} \lambda_{j} \pi(g) v_{j}=0$ for every $g \in G$, and hence $\sum_{j} \lambda_{j} w_{j}=0$ for every $\left(w_{1}, \ldots, w_{n}\right) \in V^{n}$ because $\left(v_{1}, \ldots, v_{n}\right)$ is cyclic in $V^{n}$. This leads to $\lambda_{j}=0$ for every $j$, so that the elements $v_{1}, \ldots, v_{n}$ are linearly independent.

Theorem 5.13. Let $G$ be a Lie group and $D, E \in \mathcal{D}^{\prime}(G)$ be positive definite distributions. Then the corresponding gaussian measures $\gamma_{D}$ and $\gamma_{E}$ on $\mathcal{D}^{\prime}(G)$ are equivalent if and only if the following conditions are satisfied

(i) $D$ can be written as an orthogonal sum $D=D_{0}+\sum_{n \in J} D_{n}$, where $J \subseteq \mathbb{N}$ and the representation on the subspaces $\mathcal{H}_{D_{n}}, n \in J$, are finite-dimensional isotypic and mutually disjoint.

(ii) $E=D_{0}+\sum_{n \in J} E_{n}$ with $\mathcal{H}_{D_{n}}=\mathcal{H}_{E_{n}}$, and there exist intertwining operators $T_{0}=\mathrm{id} \mathcal{H}_{D_{0}}$ and $T_{n} \in B_{G}\left(\mathcal{H}_{D_{n}}\right)$ with $T_{n} D_{n}=E_{n}$ in $\mathcal{H}_{D_{n}}^{-\infty}=\mathcal{H}_{D_{n}}$ and $\sum_{n \in J}\left\|T_{n} T_{n}^{*}-\mathbf{1}\right\|_{2}^{2}<\infty$.

Proof. We shall use Theorem 5.7. If $\gamma_{D} \sim \gamma_{E}$, then $\mathcal{H}_{D}=\mathcal{H}_{E} \subseteq \mathcal{D}^{\prime}(G)$ and the identity map $T: \mathcal{H}_{D} \rightarrow \mathcal{H}_{E}, f \mapsto f$ is a $G$-equivariant operator, so that $T^{*} T \in B\left(\mathcal{H}_{D}\right)$ is a $G$-intertwining operator. The requirement that $T^{*} T-\mathbf{1}$ is Hilbert-Schmidt implies that its range is a sum of finite-dimensional subrepresentations. In view of the preceding lemma, it can be written as $\oplus_{j \in J} \mathcal{H}_{j}$, where the $\mathcal{H}_{j}$ are isotypic, finite-dimensional and mutually disjoint. Then $T_{n}:=\left.T\right|_{\mathcal{H}_{D_{n}}}$ maps $\mathcal{H}_{D_{n}}$ into itself and

$$
\left\|T T^{*}-\mathbf{1}\right\|_{2}^{2}=\sum_{n \in J}\left\|T_{n} T_{n}^{*}-\mathbf{1}\right\|_{2}^{2} .
$$

The converse implication follows from Theorem 5.7.

Corollary 5.14. If $D$ is a positive definite distribution on $G$, then the following are equivalent

(a) $\mathcal{H}_{D}$ contains no $G$-invariant subspace of finite positive dimension. 
(b) For any two different distribution vectors $E, E^{\prime} \in \mathcal{H}_{D}^{-\infty} \subseteq \mathcal{D}^{\prime}(G)$, the corresponding gaussian measures $\gamma_{E}$ and $\gamma_{E^{\prime}}$ are inequivalent.

Example 5.15. We discuss the special case $G=\mathbb{R}^{n}$. According to the Bochner-Schwartz theorem, a distribution $D \in \mathcal{D}^{\prime}(G)$ is positive definite if and only if it is the Fourier transform $D=\widehat{\mu}$ of a tempered measure $\mu$ on the dual group $\widehat{G} \cong \mathbb{R}^{d}$, and then $\mathcal{H}_{D} \cong L^{2}\left(\mathbb{R}^{d}, \mu\right)$ with the representation

$$
\left(\pi_{D}(x) f\right)(y)=e^{-i x y} f(y) .
$$

For $D=\widehat{\mu}$ and $E=\widehat{\nu}$, the equality of the corresponding Hilbert spaces is equivalent to the existence of positive constants $c_{1}, c_{2}>0$ with

$$
c_{1} \nu \leq \mu \leq c_{2} \nu,
$$

which is equivalent to the equivalence of the measures $\mu$ and $\nu$ with the additional requirement that $\delta:=\frac{d \mu}{d \nu}$ satisfies $0<c_{1} \leq \delta \leq c_{2}$ (cf. [48] or [37]). Then $L^{2}\left(\mathbb{R}^{d}, \mu\right)=L^{2}\left(\mathbb{R}^{d}, \nu\right)$, and the identity $T: L^{2}\left(\mathbb{R}^{d}, \nu\right) \rightarrow L^{2}\left(\mathbb{R}^{d}, \mu\right)$ is an isomorphism of Banach spaces. We then have $T^{*}(f)=\delta f$ and the equivalence of the gaussian measures is equivalent to the multiplication operator $M_{\delta-1}=T^{*} T-\mathbf{1}$ being a Hilbert-Schmidt operator on $L^{2}\left(\mathbb{R}^{d}, \nu\right)$ (Theorem 5.7). This is equivalent to the condition that the restriction of $\nu$ to the subset $\{\delta \neq 1\}$ is atomic, so that the values of $\delta$ in these points are defined, and the Hilbert-Schmidt condition can be expressed as

$$
\sum_{\delta(x) \neq 1}|\delta(x)-1|^{2}<\infty
$$

We conclude that $\gamma_{D} \sim \gamma_{E}$ is equivalent to $\mu=\nu$ on the complement of an at most countable set $S$ of atoms for both measures, which satisfies

$$
\sum_{s \in S}\left|\frac{\mu(\{s\})}{\nu(\{s\})}-1\right|^{2}<\infty .
$$

Remark 5.16. (a) Theorem 5.13 generalizes in an obvious way to continuous positive definite functions on a topological group $G$.

(b) Example 5.15 generalizes in the obvious fashion to positive definite functions on a locally compact abelian group, or, more generally, on a nuclear abelian group (cf. [6]).

The following theorem covers in particular the case of one-dimensional Lie groups.

Theorem 5.17. Let $A$ be a selfadjoint operator on the Hilbert space $\mathcal{H}$. Then the following are equivalent:

(a) The gaussian measure $\gamma_{\mathcal{H}}$ can be realized on $\mathcal{H}^{-\infty}(A)$, the dual space of

$$
\mathcal{H}^{\infty}(A)=\bigcap_{n \in \mathbb{N}_{0}} \mathcal{D}\left(A^{n}\right)
$$

(b) There exists an $N \in \mathbb{N}$ such that the bounded operator $\left(\mathbf{1}+A^{2}\right)^{-N}$ is Hilbert-Schmidt.

(c) The Fréchet space $\mathcal{H}^{\infty}(A)$ is nuclear.

Proof. (a) $\Rightarrow$ (b) Let $E \subseteq \mathbb{R}$ be a bounded subset, $\mathcal{H}_{1}:=P(E) \mathcal{H}$ (for the spectral measure $P$ of $A$ ) and $\mathcal{H}_{2}:=\mathcal{H}_{1}^{\perp}$. Then we accordingly have $A=A_{1} \oplus A_{2}$, where the operator $A_{1}$ is bounded and $\mathcal{D}(A)=\mathcal{H}_{1} \oplus \mathcal{D}\left(A_{2}\right)$. This implies that

$$
\mathcal{H}^{\infty}(A)=\mathcal{H}_{1} \oplus \mathcal{H}_{2}^{\infty}\left(A_{2}\right) \quad \text { and thus } \quad \mathcal{H}^{-\infty}(A)=\mathcal{H}_{1} \oplus \mathcal{H}_{2}^{-\infty}\left(A_{2}\right) .
$$


Therefore $\gamma_{\mathcal{H}}$ can be realized on $\mathcal{H}^{-\infty}$ if and only if $\gamma_{\mathcal{H}_{1}}$ can be realized on $\mathcal{H}_{1}$, which means that $\mathcal{H}_{1}$ is finite-dimensional, and $\gamma_{\mathcal{H}_{2}}$ can be realized on $\mathcal{H}_{2}^{-\infty}\left(A_{2}\right)$.

Therefore (a) implies that all spectral projections $P([a, b]), a<b$, have finite-dimensional range. As a consequence, $A$ is diagonalizable with discrete spectrum and finite-dimensional eigenspaces; in particular $\mathcal{H}$ is separable. Let $\left(\lambda_{n}\right)_{n \in \mathbb{N}}$ be the eigenvalues of $A$, counted with multiplicities and $\left(e_{n}\right)_{n \in \mathbb{N}}$ an ONB of $\mathcal{H}$ with $A e_{n}=\lambda_{n} e_{n}$ for every $n \in \mathbb{N}$. Now

$$
\mathcal{H}^{-\infty}(A)=\left\{\sum_{n} x_{n} e_{n}:(\exists N \in \mathbb{N}) \sum_{n}\left(1+\lambda_{n}^{2}\right)^{-2 N}\left|x_{n}\right|^{2}<\infty\right\}
$$

is the union of the subspaces

$$
\mathcal{H}^{-2 N}(A)=\left\{\sum_{n} x_{n} e_{n}: \sum_{n}\left(1+\lambda_{n}^{2}\right)^{-2 N}\left|x_{n}\right|^{2}<\infty\right\} .
$$

We realize the gaussian measure $\gamma_{\mathcal{H}}$ on the product space $\mathbb{C}^{\mathbb{N}}$. Then every subspace $\mathcal{H}^{-2 N}(A)$ is measurable, and

$$
1=\gamma_{\mathcal{H}}\left(\mathcal{H}^{-\infty}(A)\right)=\lim _{N \rightarrow \infty} \gamma_{\mathcal{H}}\left(\mathcal{H}^{-2 N}(A)\right)
$$

implies that $\gamma_{\mathcal{H}}\left(\mathcal{H}^{-2 N}(A)\right)>0$ for some $N \in \mathbb{N}$. From the example in [68, p. 153], it now follows that

$$
\left\|\left(\mathbf{1}+A^{2}\right)^{-N}\right\|_{2}^{2}=\sum_{n}\left(1+\lambda_{n}^{2}\right)^{-2 N}<\infty
$$

(see also [17, Theorem 5.2]).

(b) $\Leftrightarrow(\mathrm{c})$ : The space $\mathcal{D}^{\infty}(A):=\bigcap_{n \in \mathbb{N}} \mathcal{D}\left(A^{n}\right)$ coincides with the space $\mathcal{D}^{\infty}(B)$ for $B:=\mathbf{1}+A^{2}$, and $B$ has the additional property that $B^{n} \leq B^{n+1}$ for $n \in \mathbb{N}_{0}$. The topology on $\mathcal{D}^{\infty}(B)$ is defined by the seminorms $p_{n}(v):=\left\|B^{n} v\right\|, n \in \mathbb{N}_{0}$. In view of [65, Definition 50.1], the nuclearity of the space $\mathcal{H}^{\infty}=\mathcal{D}^{\infty}(B)$ is equivalent to the condition that, for every $n \in \mathbb{N}$, there exists an $m>n$, such that the natural map $\mathcal{D}^{m}(B) \rightarrow \mathcal{D}^{n}(B)$ is nuclear. Since this map can be identified with the map $B^{n-m}: \mathcal{H} \rightarrow \mathcal{H}$, we see that the nuclearity of $\mathcal{H}^{\infty}$ is equivalent to the existence of some nuclear power of $B^{-1}$, which is equivalent to the existence of some power which is Hilbert-Schmidt. This means that $\mathcal{D}^{\infty}(A)$ is nuclear if and only if some operator $\left(\mathbf{1}+A^{2}\right)^{-N}$, $N \in \mathbb{N}$, is Hilbert-Schmidt.

(c) $\Rightarrow$ (a) follows from the Bochner-Minlos theorem.

Example 5.18. (a) In the context of reflection positivity on curved spacetimes, a natural class of selfadjoint operators arises as follows [31,32]. We call a connected complete orientable Riemannian manifold $(M, g)$ a quantizable static space-time if there exists a complete Killing vector field $\xi$ orthogonal to a hypersurface $\Sigma \subseteq M$ such that all integral curves of $\xi$ intersect $\Sigma$ exactly once. Then the flow of $\xi$ induces an isometry $\Phi: \mathbb{R} \times \Sigma \rightarrow M$ of Riemannian manifolds and $\theta(\Phi(t, x))=\Phi(-t, x)$ is an isometric involution exchanging the two open subsets $\Omega_{ \pm}=$ $\{\Phi(t, x): \pm t>0, x \in \Sigma\}$. Let $\Delta$ be the Laplacian of $(M, g)$ and $m>0$. Then the free covariance operator $C:=\left(m^{2}-\Delta\right)^{-1}$ is a bounded selfadjoint operator on $L^{2}(M)$. For $s \in \mathbb{R}$, we write $\mathcal{H}_{s}$ for the completion of $L^{2}(M)$ w.r.t. the scalar product $\langle f, g\rangle:=\left\langle f, C^{-s} g\right\rangle_{L^{2}(M)}$ (the Sobolev spaces) and obtain a Fréchet space

$$
\mathcal{S}:=\mathcal{D}^{\infty}\left(m^{2}-\Delta\right)=\mathcal{D}^{\infty}(\Delta)=\bigcap_{s \in \mathbb{R}} \mathcal{H}_{s}
$$


but in general this space is not nuclear because the Laplacian may have continuous spectrum (which contradicts the statement in [32, Definition 2.1]). But if $\Sigma$ is compact and

$$
H:=\frac{1}{2}+\frac{1}{2}\left(Q_{t}^{2}-\Delta\right),
$$

where $Q_{t}$ is the multiplication with the global time function, then there exists a power $(\mathbf{1}+H)^{-N}$ which is trace class, so that

$$
\mathcal{S}:=\mathcal{D}^{\infty}(H) \subseteq L^{2}(M)
$$

is a nuclear space. The operator $H$ is the sum of the Hamiltonian of the harmonic oscillator in the time direction and the Laplacian of $\Sigma$ [4, Proposition 7.5]. We refer to [4, Section 7.4] for a discussion of the corresponding gaussian measures on the dual space $\mathcal{S}^{\prime}$.

(b) For $M=\mathbb{R}^{d}$ and $\mathcal{H}=L^{2}\left(\mathbb{R}^{d}\right)$, the Hamiltonian of the $d$-fold harmonic oscillator

$$
H=\frac{1}{2} \sum_{j=1}^{d} Q_{j}^{2}-\partial_{j}^{2}, \quad\left(Q_{j} f\right)(x)=x_{j} f(x), \quad\left(\partial_{j} f\right)(x)=\frac{\partial}{\partial x_{j}} f(x)
$$

leads to $\mathcal{D}^{\infty}(H)=\mathcal{S}\left(\mathbb{R}^{d}\right)$ which also is a nuclear space.

Corollary 5.19. Let $(\pi, \mathcal{H})$ be a continuous unitary representation of the finite-dimensional Lie group $G$ and put $\Delta=\sum_{j} X_{j}^{2}$ for a basis $X_{1}, \ldots, X_{n}$ of $\mathfrak{g}$. Then the following are equivalent:

(a) The gaussian measure $\gamma_{\mathcal{H}}$ can be realized on $\mathcal{H}^{-\infty}$.

(b) There exists an $N \in \mathbb{N}$ such that $(\mathbf{1}-\overline{\mathrm{d} \pi(\Delta)})^{-N}$ is a Hilbert-Schmidt operator.

(c) The Fréchet space $\mathcal{H}^{\infty}$ is nuclear.

(d) $\pi$ is trace class, i.e., for each $f \in C_{c}^{\infty}(G)$, the operator $\pi(f)$ is trace class.

Proof. In view of Theorem 5.17, the equivalence of (a)-(c) follows from Nelson's theorem asserting that $\mathcal{H}^{\infty}=\mathcal{H}^{\infty}(A)$ holds for the selfadjoint operator $A:=\overline{\mathrm{d} \pi(\Delta)}$ [67, Theorem 4.4.4.5]. For the equivalence with (d) we refer to [15, Proposition 1.11].

Proposition 5.20. Let $(\pi, \mathcal{H})$ be a unitary representation of the Lie group $G$. Then the space $\mathcal{H}^{\infty}$ is nuclear in the following cases:

(a) If $G$ is compact and $\pi$ is distribution cyclic.

(b) If $G$ is connected semisimple and $\pi$ is irreducible.

(c) If $G$ is connected nilpotent and $\pi$ is irreducible.

Proof. (a) (Sketch) We denote irreducible representation with highest weight $\lambda$ by $\left(\pi_{\lambda}, V_{\lambda}\right)$. We also set $d(\lambda):=\operatorname{dim} V_{\lambda}$. We have $\mathcal{H}=\oplus_{\lambda} \mathcal{H}_{\lambda}$, where $\mathcal{H}_{\lambda}$ is the isotypic subspace of highest weight $\lambda$. The multiplicity of the simple $G$-module $V_{\lambda}$ in $\mathcal{H}_{\lambda}$ is bounded by $d(\lambda)$ (Lemma 5.12). We can then choose the basis $X_{j}$ such that $-\Delta$ coincides with the Casimir element of $\mathfrak{g}$. Let $\rho=\frac{1}{2} \sum_{\beta \in \Delta^{+}} \beta$. Then

$$
-\left.\mathrm{d} \pi(\Delta)\right|_{\mathcal{H}_{\lambda}}=\left(\|\lambda+\rho\|^{2}-\|\rho\|^{2}\right) \mathbf{1}
$$

by Freudenthal's lemma [48, Lemma IX.5.2]. We therefore have

$$
\begin{aligned}
\left\|(\mathbf{1}-\mathrm{d} \pi(\Delta))^{-N}\right\|_{2}^{2} & =\sum_{\lambda}\left(1+\|\lambda+\rho\|^{2}-\|\rho\|^{2}\right)^{-2 N} \operatorname{dim} \mathcal{H}_{\lambda} \\
& \leq \sum_{\lambda}\left(1+\|\lambda+\rho\|^{2}-\|\rho\|^{2}\right)^{-2 N} d(\lambda)^{2} .
\end{aligned}
$$


This expression is finite for some $N \in \mathbb{N}$ because $d(\lambda)$ is bounded by a polynomial in $\|\lambda\|[67$, Lemma 4.4.2.3]. This means that there exists an $N \in \mathbb{N}$ for which $(\mathbf{1}-\mathrm{d} \pi(\Delta))^{-N}$ is HilbertSchmidt. Therefore $\mathcal{H}^{\infty}=\mathcal{D}^{\infty}(\mathrm{d} \pi(\Delta))=\mathcal{D}^{\infty}\left((\mathbf{1}-\mathrm{d} \pi(\Delta))^{N}\right)$ is nuclear.

(b) ([13, Theorem 2.1]) Write $\mathfrak{g}=\mathfrak{k} \oplus \mathfrak{s}$ for the Cartan decomposition of $\mathfrak{g}$. Then we can choose the basis $X_{j}$ such that $\Delta=\Omega+2 \Delta_{\mathfrak{k}}$ where $\Omega$ is the Casimir element and $\Delta_{\mathfrak{k}}$ is the Laplacian for $\mathfrak{k}$. As $\Omega$ acts by a scalar and the dimension of $\mathcal{H}_{\lambda}$ is bounded by $d(\lambda)^{2}$, the claim follows as in (a).

(c) ([13, Proposition 1.9(a)]) According to [39, Theorem 3, p. 103], we can realize $\pi$ in $L^{2}\left(\mathbb{R}^{n}, d x\right)$ such that $\mathcal{H}^{\infty}$ coincides with the Schwartz space $\mathcal{S}\left(\mathbb{R}^{n}\right)$ which is nuclear.

Example 5.21. For the distribution $D=\delta_{1}$ on the compact Lie group $G$, the corresponding representation is the regular representation on $\mathcal{H}_{D} \cong L^{2}(G)$. In this case $L^{2}(G)^{\infty}=C^{\infty}(G)$ (f.i. by the Dixmier-Malliavin theorem) is a nuclear space and $L^{2}(G)^{-\infty}=C^{-\infty}(G)$ is the space of distributions on $G$. In particular, the measure $\gamma_{D}$ can be realized in this space.

Remark 5.22. The condition $\mathcal{H}^{\infty}=\mathcal{H}$ is equivalent to the smoothness of the representation, which in turn is equivalent to the boundedness of the operators $\mathrm{d} \pi(X), X \in \mathfrak{g}$. If this is the case, then $\mathcal{H}^{-\infty}=\mathcal{H}$. If $\mathcal{H}$ is infinite-dimensional it is not nuclear, and since $\mathcal{H} \subseteq \mathcal{H}^{a}$ (the algebraic dual) is a zero set for $\gamma_{\mathcal{H}}$, the corresponding gaussian measure can not be realized on $\mathcal{H}^{-\infty}$.

Examples arise from the multiplication representation of an abelian Lie group $G$ on the space $\mathcal{H}=L^{2}(\widehat{G}, \mu)$, where $\mu$ is a compactly supported measure on the dual group $\widehat{G}=\operatorname{Hom}(G, \mathbb{T})$. These representations are smooth and generated by the cyclic vector 1 . If $\mathcal{H}$ is infinitedimensional, then it is not nuclear. In particular, $\gamma_{D}$ is not realized on the subspace $\mathcal{H}_{D}^{-\infty}$ of $\mathcal{D}^{\prime}(G)$.

Example 5.23. The multiplication representation of $G=\mathbb{R}$ on $L^{2}(\mathbb{R}, \mu)$ for the measure $\mu=$ $\sum_{n \in \mathbb{N}} \frac{1}{2^{n}} \delta_{\frac{1}{n}}$ is norm continuous and $\mathcal{H}^{\infty}=\mathcal{H}$ is not nuclear.

\subsection{Ergodicity of $\gamma_{\mathcal{H}}$ for $G$}

Let $(\pi, \mathcal{H})$ be an orthogonal representation of $G$ on the real Hilbert space $\mathcal{H}$ and $\gamma_{\mathcal{H}}$ the corresponding gaussian measure. We consider the so-called gaussian action of $G$ on the gaussian probability space $\left(\mathcal{H}^{a}, \gamma_{\mathcal{H}}\right)$ on the algebraic dual $\mathcal{H}^{a}$. The measure $\gamma_{\mathcal{H}}$ is $G$-ergodic if and only if

$$
\Gamma(\mathcal{H})^{G}=L^{2}\left(\mathcal{H}^{a}, \gamma_{\mathcal{H}}\right)^{G}=\mathbb{C} 1 .
$$

In this section we derive a criterion for this condition to be satisfied.

Lemma 5.24. Let $\left(\pi_{j}, \mathcal{H}_{j}\right)_{j=1,2}$ be two orthogonal representations of the group $G$.

(i) If $\mathcal{H}_{1}$ has no non-zero finite-dimensional subrepresentations, then all finite-dimensional invariant subspaces of $\mathcal{H}_{1} \otimes \mathcal{H}_{2}$ are zero. In particular, $\left(\mathcal{H}_{1} \otimes \mathcal{H}_{2}\right)^{G}=\{0\}$.

(ii) The subspace $\left(\mathcal{H}_{1} \otimes \mathcal{H}_{2}\right)_{f}$ generated by the finite-dimensional invariant subspaces coincides with $\mathcal{H}_{1, f} \otimes \mathcal{H}_{2, f}$.

Proof. (i) First we show that the subspace $\left(\mathcal{H}_{1} \otimes \mathcal{H}_{2}\right)^{G}$ of fixed vectors is trivial. Since $\left(\mathcal{H}_{1} \otimes\right.$ $\left.\mathcal{H}_{2}\right)^{G}$ can be identified with the space of Hilbert-Schmidt intertwining operators $A: \mathcal{H}_{2}^{*} \rightarrow \mathcal{H}_{1}$, any such operator leads to the self intertwining operator $A A^{*} \in B_{2}\left(\mathcal{H}_{1}\right)$ and its eigenspaces are finite-dimensional $G$-invariant subspaces, hence trivial. Let $\mathcal{F} \subseteq \mathcal{H}_{1} \otimes \mathcal{H}_{2}$ be a finite-dimensional invariant subspace. Then $\operatorname{id}_{\mathcal{F}} \in \mathcal{F} \otimes \mathcal{F}^{*} \subseteq \mathcal{H}_{1} \otimes \mathcal{H}_{2} \otimes \mathcal{H}_{1}^{*} \otimes \mathcal{H}_{2}^{*}$ is a fixed vector, so that $\mathcal{F}=\{0\}$ follows from the preceding argument.

(ii) We write

$$
\mathcal{H}_{1} \otimes \mathcal{H}_{2}=\left(\mathcal{H}_{1, f} \otimes \mathcal{H}_{2, f}\right) \oplus\left(\mathcal{H}_{1, f}^{\perp} \otimes \mathcal{H}_{2}\right) \oplus\left(\mathcal{H}_{1, f} \otimes \mathcal{H}_{2, f}^{\perp}\right)
$$


and apply the preceding proposition to see that only the first summand contains non-zero finitedimensional invariant subspaces.

For unitary representations, one can also introduce the terminology from measure preserving actions on a probability space, where $\mathcal{H} \cong L_{0}^{2}(X, \Sigma, \mu)=\left\{f \in L^{2}(X, \Sigma, \mu): \int_{X} f d \mu=0\right\}$.

Definition 5.25. Let $(\pi, \mathcal{H})$ be a unitary representation and $\left(\pi^{*}, \mathcal{H}^{*}\right)$ the dual representation. We say that $\pi$ is:

(a) ergodic if $\mathcal{H}^{G}=\{0\}$,

(b) weakly mixing if $\pi \otimes \pi^{*}$ is ergodic,

(c) mixing if $G$ is locally compact and all matrix coefficients are contained in $C_{0}(G)$.

The following proposition is an elaboration of the main result of [63] whose main focus is the equivalence of (i) and (ii).

Theorem 5.26. (I.E. Segal) For an orthogonal representation $(\pi, \mathcal{H})$ of the group $G$, the following are equivalent:

(i) $\mathcal{H}$ contains no non-zero finite-dimensional invariant subspaces.

(ii) The gaussian measure $\gamma_{\mathcal{H}}$ is G-ergodic.

(iii) $\pi$ is weakly mixing.

(iv) For every orthogonal representation $(\rho, \mathcal{K})$, the representation $\pi \otimes \rho$ is ergodic.

If these conditions are satisfied, then, for every $N \in \mathbb{N}$, the product measure $\gamma_{\mathcal{H}}^{\otimes N}$ is also ergodic.

Proof. (i) $\Leftrightarrow$ (ii): (cf. [8, Proposition A.1.12, Corollary A.7.15]) $)^{6}$ If $\mathcal{F} \subseteq \mathcal{H}$ is a finitedimensional invariant subspace, then $\gamma_{\mathcal{F}}$ (which is equivalent to Lebesgue measure on $\mathcal{F}$ ) is not ergodic. Now $\gamma_{\mathcal{H}} \cong \gamma_{\mathcal{F}} \otimes \gamma_{\mathcal{F} \perp}$ implies that $\gamma_{\mathcal{H}}$ is not ergodic.

If, conversely, all finite-dimensional invariant subspaces of $\mathcal{H}$ are trivial, then all $G$-fixed vectors in $S^{n}(\mathcal{H}) \subseteq \mathcal{H}^{\otimes n}$ are trivial if $n>0$ (Lemma 5.24). Hence the assertion follows from the $G$-equivariant decomposition $\Gamma(\mathcal{H}) \cong \mathbb{C} \Omega \oplus \bigoplus_{n>0} S^{n}(\mathcal{H})$.

(i) $\Rightarrow$ (iv) follows from Lemma 5.24(i).

(iv) $\Rightarrow$ (iii) is trivial.

(iii) $\Rightarrow$ (i): If $\mathcal{F} \subseteq \mathcal{H}$ is a finite-dimensional invariant subspace, then $\operatorname{id}_{\mathcal{F}} \subseteq \mathcal{F} \otimes \mathcal{F}^{*} \cong \mathcal{H} \otimes \mathcal{H}^{*}$ is a fixed vector.

If (i) is satisfied, then the canonical representation on $\mathcal{H}^{N}$ also contains no non-zero finitedimensional invariant subspace, so that the $G$-action on the product spaces $\Gamma\left(\mathcal{H}^{N}\right) \cong \Gamma(\mathcal{H})^{N}$ with the product measure $\gamma_{\mathcal{H}^{N}} \cong \gamma_{\mathcal{H}}^{\otimes N}$ is also ergodic.

Remark 5.27. Let $\mathcal{H}$ be a complex Hilbert space and $(\pi, \mathcal{H})$ be a unitary representation of $G$ on $\mathcal{H}$. We write $\mathcal{H}^{\mathbb{R}}$ for the underlying real Hilbert space and $\mathcal{F}_{\mathbb{C}}(\mathcal{H})$ for the associated Fock space over $\mathbb{C}$. Then

$$
\Gamma\left(\mathcal{H}^{\mathbb{R}}\right) \cong \mathcal{F}_{\mathbb{C}}\left(\left(\mathcal{H}^{\mathbb{R}}\right)_{\mathbb{C}}\right) \cong \mathcal{F}_{\mathbb{C}}(\mathcal{H} \oplus \overline{\mathcal{H}}) \cong \mathcal{F}_{\mathbb{C}}(\mathcal{H}) \otimes_{\mathbb{C}} \mathcal{F}_{\mathbb{C}}(\overline{\mathcal{H}}) \cong B_{2}\left(\mathcal{F}_{\mathbb{C}}(\mathcal{H})\right)
$$

Therefore the requirement that $\mathcal{F}_{\mathbb{C}}(\mathcal{H})^{G}=\mathbb{C} \Omega$ is weaker than the ergodicity of the measure $\gamma_{\mathcal{H}}$.

Let $\mathcal{H}=\mathcal{H}_{f} \oplus \mathcal{H}_{f}^{\perp}$ denote the decomposition into the closed subspace $\mathcal{H}_{f}$ generated by all finite-dimensional invariant subspaces and its orthogonal complement $\mathcal{H}_{f}^{\perp}$. ${ }^{7}$ Then

$$
\mathcal{F}_{\mathbb{C}}(\mathcal{H}) \cong \mathcal{F}_{\mathbb{C}}\left(\mathcal{H}_{f}\right) \otimes \mathcal{F}_{\mathbb{C}}\left(\mathcal{H}_{f}^{\perp}\right)
$$

\footnotetext{
${ }^{6}$ We thank Bachir Bekka for this reference.

${ }^{7}$ Note that the representation on $\mathcal{H}_{f}$ factors through a representation of a compact group.
} 
and Lemma 5.24 implies that the subspace $\mathcal{F}_{\mathbb{C}}\left(\mathcal{H}_{f}\right) \otimes \bigoplus_{n>0} S^{n}\left(\mathcal{H}_{f}^{\perp}\right)$ contains no non-zero fixed vectors. Therefore $\mathcal{F}_{\mathbb{C}}(\mathcal{H})^{G}=\mathcal{F}_{\mathbb{C}}\left(\mathcal{H}_{f}\right)^{G}$.

If $G$ is abelian, then $\mathcal{H}_{f}$ is spanned by eigenvectors for certain characters $X \subseteq \widehat{G}$, and the corresponding characters of $S^{n}\left(\mathcal{H}_{f}\right)$ are the finite products $\chi_{1} \cdots \chi_{n}, \chi_{j} \in X$. Therefore $\mathcal{F}_{\mathbb{C}}(\mathcal{H})^{G}=\mathbb{C} \Omega$ is equivalent to the condition that all products $\chi_{1} \cdots \chi_{n}, \chi_{j} \in X, n>0$, are non-trivial.

Definition 5.28 ([9, Definition 2.14]). A measure preserving action of $G$ on a finite measure space $(X, \Sigma, \mu)$ is said to be weakly mixing if the representation on the subspace

$$
L_{0}^{2}(X, \Sigma, \mu)_{0}:=1^{\perp} \subseteq L^{2}(X, \Sigma, \mu)
$$

contains no non-zero finite-dimensional invariant subspaces. In view of Theorem 5.26, this is equivalent to the representation on $L_{0}^{2}(X, \Sigma, \mu)$ to be weakly mixing in the sense of Definition 5.25 .

The following proposition justifies Definition 5.25(b).

Proposition 5.29. A measure preserving action of $G$ on $(X, \Sigma, \mu)$ is weakly mixing if and only if the corresponding action on the pair space $\left(X^{2}, \Sigma \otimes \Sigma, \mu \otimes \mu\right)$ is ergodic.

Proof. We have

$$
L^{2}(X \times X, \mu \otimes \mu)^{G} \cong\left(L^{2}(X, \mu) \otimes L^{2}(X, \mu)\right)^{G} \subseteq L^{2}(X, \mu)_{f} \otimes L^{2}(X, \mu)_{f} .
$$

If the action is weakly mixing, then $L^{2}(X, \mu)_{f}=\mathbb{C} 1$ implies that $L^{2}(X \times X, \mu \otimes \mu)^{G}=\mathbb{C} 1$, so that the product action is ergodic.

If, conversely, the product action is ergodic and $\mathcal{F} \subseteq L_{0}^{2}(X, \mu)$ is a finite-dimensional invariant subspace, then

$$
\mathcal{F} \otimes \mathcal{F}^{*} \subseteq L^{2}(X, \mu) \otimes L^{2}(X, \mu) \cong L^{2}(X \times X, \mu \otimes \mu)
$$

leads to a fixed vector in $L^{2}(X \times X, \mu \otimes \mu)$, which implies $\mathcal{F}=\{0\}$. We conclude that the action on $(X, \mu)$ is weakly mixing.

Example 5.30. If $(\pi, \mathcal{H})$ is an orthogonal representation for which the corresponding gaussian action on $\Gamma(\mathcal{H})=\left(\mathcal{H}^{a}, \gamma_{\mathcal{H}}\right)$ is ergodic, then it is weakly mixing by Theorem 5.26.

\section{A Continuity of a stochastic process on $G$}

This appendix refers to Example 3.13. Clearly, the most natural continuity requirement from the perspective of representation theory is that the representation of $G$ in $L^{2}\left(B^{G}, \mathfrak{B}^{G}, \nu\right)$ is continuous. In this subsection we collect some remarks that are useful for the verification of this continuity.

Lemma A.1. For a semigroup $\left(P_{s}\right)_{s \in S}$ of positivity preserving operators on $L^{\infty}(X, \mathfrak{S}, \mu)$, the strong continuity of the representation of $S$ on $L^{2}(X, \mathfrak{S}, \nu)$ implies continuity in measure

$$
\lim _{s \rightarrow s_{0}} \nu\left(\left|P_{s} f-P_{s_{0}} f\right| \geq \varepsilon\right)=0 \quad \text { for every } \quad \varepsilon>0
$$

Proof. This follows from $\varepsilon^{2} \nu\left(\left|P_{s} f-P_{s_{0}} f\right| \geq \varepsilon^{2}\right) \leq \int_{X}\left|P_{s} f-P_{s_{0}} f\right|^{2} d \nu \rightarrow 0$. 
Corollary A.2. For a square integrable stationary $\mathbb{R}$-valued process $\left(X_{g}\right)_{g \in G}$, the continuity of the representation of $G$ on $L^{2}\left(B^{G}, \mathfrak{B}^{G}, \nu\right)$ implies that

$$
\lim _{g \rightarrow \mathbf{1}} \nu\left(\left|X_{g}-X_{\mathbf{1}}\right| \geq \varepsilon\right)=0 \quad \text { for every } \varepsilon>0 .
$$

Remark A.3. Let $G$ be a group acting in a measure preserving way on the finite measure space $(Q, \Sigma, \mu)$. It is easy to see that the continuity of the representation on $L^{2}(Q, \Sigma, \mu)$ is equivalent to the continuity of the orbit maps of the characteristic functions, which in turn is equivalent to the continuity of the maps

$$
d_{A}: G \rightarrow \mathbb{R}, \quad d_{A}(g):=\mu((g A) \Delta A) \quad \text { for } \quad A \in \Sigma .
$$

Actually this condition is equivalent to the continuity of the $G$-action on the metric space $\left(\Sigma / J_{\mu}, d\right)$, where $d(A, B)=\mu(A \Delta B)$ and $J_{\mu}$ denotes the ideal of $\mu$-zero sets.

Next we observe that the set of all bounded functions $f \in L^{2}(Q, \Sigma, \mu)$ for which the $G$-orbit map in $L^{2}(Q, \Sigma, \mu)$ is continuous is a subalgebra. This follows immediately from the estimate

$$
\left\|U_{g}(f h)-f h\right\|_{2} \leq\|h\|_{\infty}\left\|U_{g} f-f\right\|_{2}+\|f\|_{\infty}\left\|U_{g} h-h\right\|_{2} .
$$

This implies that

$$
\Sigma_{c}:=\left\{A \in \Sigma: d_{A} \in C(G, \mathbb{R})\right\}
$$

contains $\varnothing$ and $Q$ and is stable under complements, finite intersections and finite unions. From the closedness of the subspace of continuous vectors in $L^{2}(Q, \Sigma, \mu)$ we further derive that $\Sigma_{c}$ is stable under countable unions, hence a $\sigma$-subalgebra. We conclude that it suffices to verify the continuity of the functions $d_{A}$ for a collection of subsets generating the $\sigma$-algebra $\Sigma$.

Lemma A.4. For a real-valued stationary process $\left(X_{g}\right)_{g \in G}$, the condition

$$
\lim _{g \rightarrow \mathbf{1}} \nu\left(\left|X_{g}-X_{1}\right| \geq \varepsilon\right)=0
$$

of continuity in measure implies continuity of the $G$-representation on $L^{2}(Q, \Sigma, \nu)$.

Proof. In view of the preceding remark, it suffices to show that, for the sets $A_{g_{0}}:=\left\{X_{g_{0}} \geq a\right\}$, $a \in \mathbb{R}$, the function $d_{A}(g)=\nu\left(g A_{g_{0}} \Delta A_{g_{0}}\right)=\nu\left(A_{g g_{0}} \Delta A_{g_{0}}\right)$ is continuous in $\mathbf{1}$. Note that

$$
A_{g g_{0}} \Delta A_{g_{0}} \subseteq\left\{\left|X_{g g_{0}}-X_{g_{0}}\right| \geq \delta\right\} \cup\left\{X_{g g_{0}} \in\right] a, a+\delta[\} \cup\left\{X_{g_{0}} \in\right] a, a+\delta[\} .
$$

For $\delta$ sufficiently small, the last two sets on the right have measure at most $\frac{\varepsilon}{3}$, so that

$$
\nu\left(\left\{\left|X_{g_{0}}-X_{g g_{0}}\right| \geq \delta\right\}\right)<\frac{\varepsilon}{2} \quad \text { leads to } \quad \nu\left(g A_{g_{0}} \Delta A_{g_{0}}\right)<\varepsilon .
$$

\section{B Markov kernels}

In this appendix we discuss briefly some basic properties of Markov kernels that are needed for this article.

Definition B.1 ([7, Section 36]). (a) Let $(Q, \Sigma)$ and $\left(Q^{\prime}, \Sigma^{\prime}\right)$ be measurable spaces. Then a function

$$
K: Q \times \Sigma^{\prime} \rightarrow[0, \infty]
$$

is called a kernel if 
(K1) for every $A^{\prime} \in \Sigma^{\prime}$, the function $K^{A^{\prime}}(\omega):=K\left(\omega, A^{\prime}\right)$ is $\Sigma$-measurable, and

(K2) for every $\omega \in Q$, the function $K_{\omega}\left(A^{\prime}\right):=K\left(\omega, A^{\prime}\right)$ is a (positive) measure.

A kernel is called a Markov kernel if the measures $K_{\omega}$ are probability measures.

(b) A kernel $K: Q \times \Sigma^{\prime} \rightarrow[0, \infty]$ associates to a measure $\mu$ on $(Q, \Sigma)$ the measure

$$
(\mu K)\left(A^{\prime}\right):=\int \mu(d \omega) K\left(\omega, A^{\prime}\right)
$$

on $\left(Q^{\prime}, \Sigma^{\prime}\right)$. To every measurable function $f^{\prime}: Q^{\prime} \rightarrow[0, \infty]$, it associates the function

$$
K f^{\prime}: Q \rightarrow[0, \infty], \quad\left(K f^{\prime}\right)(\omega):=\int_{Q^{\prime}} K\left(\omega, d \omega^{\prime}\right) f^{\prime}\left(\omega^{\prime}\right) .
$$

Now the Markov property corresponds to $K 1=K$ for the constant function 1 .

(c) If $\left(Q_{j}, \Sigma_{j}\right)_{j=1,2,3}$ are measurable spaces, then composition of kernels $K_{1}$ on $Q_{1} \times \Sigma_{2}$ and $K_{2}$ on $Q_{2} \times \Sigma_{3}$ is defined by

$$
\left(K_{1} K_{2}\right)\left(\omega_{1}, A_{3}\right)=\int_{Q_{2}} K_{1}\left(\omega_{1}, d \omega_{2}\right) K_{2}\left(\omega_{2}, A_{3}\right) .
$$

In particular, we obtain on a measurable space $(Q, \Sigma)$ the concept of a semigroup $\left(P_{s}\right)_{s \in S}$ of (Markov) kernels by the requirement that $P_{s} P_{t}=P_{s t}$ for $s t \in S$. Here the classical case is $S=\mathbb{R}_{+}$.

Remark B.2. (a) For $Q=Q^{\prime}$ and $\Sigma=\Sigma^{\prime}$, every Markov kernel $K$ defines a positivity preserving operator on measurable functions by

$$
(K f)(\omega):=\int_{Q} K\left(\omega, d \omega^{\prime}\right) f\left(\omega^{\prime}\right)
$$

The Markov property implies that $K 1=1$ and that $\|K f\|_{\infty} \leq\|f\|_{\infty}$.

(b) For a measure $\mu$ on $(Q, \Sigma)$ we then have

$$
\int_{Q} f d(\mu K)=\int_{Q} \int_{Q} \mu(d \omega) K\left(\omega, d \omega^{\prime}\right) f\left(\omega^{\prime}\right)=\int_{Q} K f d \mu .
$$

Therefore the relation $\mu K=\mu$ is equivalent to the invariance of $\mu$ as a functional on non-negative bounded measurable functions under the operator $f \mapsto K f$.

Remark B.3. For a Markov semigroup $\left(P_{t}\right)_{t \geq 0}$ on $(Q, \Sigma)$ and a probability measure $\mu$ on $(Q, \Sigma)$, we obtain for $0 \leq t_{1}<\cdots<t_{n}$ and $\mathbf{t}=\left(t_{1}, \ldots, t_{n}\right)$ a probability measure $P_{\mathbf{t}}^{\mu}$ on $Q^{n}[7$, Satz 36.4]:

$$
P_{\mathbf{t}}^{\mu}(B)=\int_{Q^{n+1}} \chi_{B}\left(x_{1}, \ldots, x_{n}\right) \mu\left(d x_{0}\right) P_{t_{1}}\left(x_{0}, d x_{1}\right) P_{t_{2}-t_{1}}\left(x_{1}, d x_{2}\right) \cdots P_{t_{n}-t_{n-1}}\left(x_{n-1}, d x_{n}\right) .
$$

The measure $P_{\mathbf{t}}^{\mu}$ can also be written as $P_{\mathbf{t}}^{\mu}=\mu P_{\mathbf{t}}$ for the kernel

$$
P_{\mathbf{t}}\left(x_{0}, B\right)=\int_{Q^{n}} \chi_{B}\left(x_{1}, \ldots, x_{n}\right) P_{t_{1}}\left(x_{0}, d x_{1}\right) P_{t_{2}-t_{1}}\left(x_{1}, d x_{2}\right) \cdots P_{t_{n}-t_{n-1}}\left(x_{n-1}, d x_{n}\right)
$$

on $Q \times \Sigma^{n}$. This is a projective family of measures. If $(Q, \Sigma)$ is a polish space, then this leads to a stochastic process $\left(X_{t}\right)_{t \geq 0}$ with state space $(Q, \Sigma)$ [7, Corollary 35.4]. The probability measure $\mu$ is the distribution of $X_{0}$. It is called the initial distribution. According to [7, Theorem 42.3], the so obtained process has the Markov property. 


\section{Extending stationary Markov processes to the real line}

Let $\left(P_{t}\right)_{t \geq 0}$ be a Markov semigroup on $(Q, \Sigma)$ and $\nu$ be a measure on $(Q, \Sigma)$. We obtain for

$$
-s_{m}<-s_{m-1}<\cdots<-s_{1}<0 \leq t_{1}<\cdots<t_{n}
$$

a measure $P_{\mathbf{s}, \mathbf{t}}^{\nu}$ on $Q^{m+n}$ by

$$
\begin{aligned}
P_{\mathbf{s}, \mathbf{t}}^{\nu}(B)= & \int_{Q^{n+m+1}} \chi_{B}\left(y_{m}, \ldots, y_{1}, x_{1}, \ldots, x_{n}\right) \\
& \times P_{s_{m}-s_{m-1}}\left(y_{m-1}, d y_{m}\right) \cdots P_{s_{2}-s_{1}}\left(y_{1}, d y_{2}\right) P_{s_{1}}\left(x_{0}, d y_{1}\right) \nu\left(d x_{0}\right) \\
& \times P_{t_{1}}\left(x_{0}, d x_{1}\right) P_{t_{2}-t_{1}}\left(x_{1}, d x_{2}\right) \cdots P_{t_{n}-t_{n-1}}\left(x_{n-1}, d x_{n}\right) .
\end{aligned}
$$

This means that $P_{\mathbf{s}, \mathbf{t}}^{\nu}=\nu P_{\mathbf{s}, \mathbf{t}}$ for the kernel

$$
\begin{aligned}
P_{\mathbf{s}, \mathbf{t}}\left(x_{0}, B\right)= & \int_{Q^{n+m}} \chi_{B}\left(y_{m}, \ldots, y_{1}, x_{1}, \ldots, x_{n}\right) \\
& \times P_{s_{m}-s_{m-1}}\left(y_{m-1}, d y_{m}\right) \cdots P_{s_{2}-s_{1}}\left(y_{1}, d y_{2}\right) P_{s_{1}}\left(x_{0}, d y_{1}\right) \\
& P_{t_{1}}\left(x_{0}, d x_{1}\right) P_{t_{2}-t_{1}}\left(x_{1}, d x_{2}\right) \cdots P_{t_{n}-t_{n-1}}\left(x_{n-1}, d x_{n}\right) .
\end{aligned}
$$

This is a projective family of measures. If $(Q, \Sigma)$ is a polish space, this leads to a stochastic process $\left(X_{t}\right)_{t \in \mathbb{R}}$ with state space $(Q, \Sigma)$ [7, Corollary 35.4]. If $\nu$ is a probability measure, then the measure $\nu$ is called the initial distribution of the process. It coincides with the distribution of $X_{0}$. Suppose, in addition, that

$$
\begin{aligned}
\int_{Q^{2}} g(\omega) \nu(d \omega) P_{t}\left(\omega, d \omega^{\prime}\right) f\left(\omega^{\prime}\right) & =\int_{Q}\left(P_{t} f\right) g \nu\left(d x_{0}\right)=\int_{Q} f\left(P_{t} g\right) \nu\left(d x_{0}\right) \\
& =\int_{Q^{2}} g(\omega) \nu\left(d \omega^{\prime}\right) P_{t}\left(\omega^{\prime}, d \omega\right) f\left(\omega^{\prime}\right) \quad \text { for } \quad 0 \leq f, g .
\end{aligned}
$$

Then

$$
\begin{aligned}
P_{\mathbf{s}, \mathbf{t}}^{\nu}(B)= & \int_{Q^{n+m+1}} \chi_{B}\left(y_{m}, \ldots, y_{1}, x_{1}, \ldots, x_{n}\right) \\
& \times P_{s_{m}-s_{m-1}}\left(y_{m-1}, d y_{m}\right) \cdots P_{s_{2}-s_{1}}\left(y_{1}, d y_{2}\right) P_{s_{1}}\left(x_{0}, d y_{1}\right) \nu\left(d x_{0}\right) \\
& \times P_{t_{1}}\left(x_{0}, d x_{1}\right) P_{t_{2}-t_{1}}\left(x_{1}, d x_{2}\right) \cdots P_{t_{n}-t_{n-1}}\left(x_{n-1}, d x_{n}\right) \\
= & \int_{Q^{n+m+1}} \chi_{B}\left(y_{m}, \ldots, y_{1}, x_{1}, \ldots, x_{n}\right) \\
& \times P_{s_{m}-s_{m-1}}\left(y_{m-1}, d y_{m}\right) \cdots P_{s_{2}-s_{1}}\left(y_{1}, d y_{2}\right) \nu\left(d y_{1}\right) P_{s_{1}}\left(y_{1}, d x_{0}\right) \\
& \times P_{t_{1}}\left(x_{0}, d x_{1}\right) P_{t_{2}-t_{1}}\left(x_{1}, d x_{2}\right) \cdots P_{t_{n}-t_{n-1}}\left(x_{n-1}, d x_{n}\right) \\
= & \int_{Q^{n+m}} \chi_{B}\left(y_{m}, \ldots, y_{1}, x_{1}, \ldots, x_{n}\right) \\
& \times P_{s_{m}-s_{m-1}}\left(y_{m-1}, d y_{m}\right) \cdots P_{s_{2}-s_{1}}\left(y_{1}, d y_{2}\right) \nu\left(d y_{1}\right) P_{s_{1}+t_{1}}\left(y_{1}, d x_{1}\right) \\
& \times P_{t_{2}-t_{1}}\left(x_{1}, d x_{2}\right) \cdots P_{t_{n}-t_{n-1}}\left(x_{n-1}, d x_{n}\right) \\
= & \int_{Q^{n+m}} \chi_{B}\left(y_{m}, \ldots, y_{1}, x_{1}, \ldots, x_{n}\right) \\
& \times P_{s_{m}-s_{m-1}}\left(y_{m-1}, d y_{m}\right) \cdots \nu\left(d y_{2}\right) P_{s_{2}-s_{1}}\left(y_{2}, d y_{1}\right) P_{s_{1}+t_{1}}\left(y_{1}, d x_{1}\right) \\
& \times P_{t_{2}-t_{1}}\left(x_{1}, d x_{2}\right) \cdots P_{t_{n}-t_{n-1}}\left(x_{n-1}, d x_{n}\right)=\cdots \\
= & \int_{Q^{n+m}} \chi_{B}\left(y_{m}, \ldots, y_{1}, x_{1}, \ldots, x_{n}\right)
\end{aligned}
$$




$$
\begin{aligned}
& \times \nu\left(d y_{m}\right) P_{s_{m}-s_{m-1}}\left(y_{m}, d y_{m-1}\right) \cdots P_{s_{2}-s_{1}}\left(y_{2}, d y_{1}\right) P_{s_{1}+t_{1}}\left(y_{1}, d x_{1}\right) \\
& \times P_{t_{2}-t_{1}}\left(x_{1}, d x_{2}\right) \cdots P_{t_{n}-t_{n-1}}\left(x_{n-1}, d x_{n}\right) .
\end{aligned}
$$

If $\nu$ is a probability measure, we thus obtain a stationary process with values in $Q$. For $\mathbf{t}=$ $\left(t_{1}, \ldots, t_{n}\right)$ and $t_{1}<\ldots<t_{n}$ in $\mathbb{R}$, we then have for the distribution of this process

$$
P_{\mathbf{t}}^{\nu}(B)=\int_{Q^{n}} \chi_{B}\left(x_{1}, \ldots, x_{n}\right) \nu\left(d x_{1}\right) P_{t_{2}-t_{1}}\left(x_{1}, d x_{2}\right) \cdots P_{t_{n}-t_{n-1}}\left(x_{n-1}, d x_{n}\right) .
$$

This formula immediately implies that the translation invariance of the measure $P^{\nu}$ on $Q^{\mathbb{R}}$.

\section{Acknowledgements}

The research of P. Jorgensen was partially supported by the Binational Science Foundation Grant number 2010117. The research of K.-H. Neeb was supported by DFG-grant NE 413/7-2, Schwerpunktprogramm "Darstellungstheorie". The research of G. Ólafsson was supported by NSF grant DMS-1101337. The authors wish to thank the Mathematisches Forschungsinstitut Oberwolfach for hosting a Workshop on "Reflection Positivity in Representation Theory, Stochastics and Physics" November, 30 - December 6, 2014. The present research was started at the workshop, and it has benefitted from our discussions with the participants there.

\section{References}

[1] Albeverio S., Driver B.K., Gordina M., Vershik A.M., Equivalence of the Brownian motion and energy representations, arXiv:1511.07378.

[2] Alpay D., Jorgensen P.E.T., Stochastic processes induced by singular operators, Numer. Funct. Anal. Optim. 33 (2012), 708-735, arXiv:1109.5273.

[3] Alpay D., Jorgensen P.E.T., Levanony D., A class of Gaussian processes with fractional spectral measures, J. Funct. Anal. 261 (2011), 507-541, arXiv:1009.0233.

[4] Anderson C.C., Defining physics at imaginary time: reflection positivity for certain Riemannian manifolds, Thesis, Harvard University, 2013, available at http://www.math.harvard.edu/theses/senior/anderson/ anderson.pdf.

[5] Applebaum D., Stochastic evolution of Yang-Mills connections on the noncommutative two-torus, Lett. Math. Phys. 16 (1988), 93-99.

[6] Banaszczyk W., Additive subgroups of topological vector spaces, Lecture Notes in Math., Vol. 1466, SpringerVerlag, Berlin, 1991.

[7] Bauer H., Probability theory, de Gruyter Studies in Mathematics, Vol. 23, Walter de Gruyter \& Co., Berlin, 1996.

[8] Bekka B., de la Harpe P., Valette A., Kazhdan's property (T), New Mathematical Monographs, Vol. 11, Cambridge University Press, Cambridge, 2008.

[9] Bekka B., Mayer M., Ergodic theory and topological dynamics of group actions on homogeneous space, London Mathematical Society Lecture Note Series, Vol. 269, Cambridge University Press, Cambridge, 2000.

[10] Bendikov A., Saloff-Coste L., Brownian motions on compact groups of infinite dimension, in Heat Kernels and Analysis on Manifolds, Graphs, and Metric Spaces (Paris, 2002), Contemp. Math., Vol. 338, Amer. Math. Soc., Providence, RI, 2003, 41-63.

[11] Bogachev V.I., Gaussian measures, Mathematical Surveys and Monographs, Vol. 62, Amer. Math. Soc., Providence, RI, 1998.

[12] Bratteli O., Robinson D.W., Operator algebras and quantum statistical mechanics. I. $C^{*}$ - and $W^{*}$-algebras. Symmetry groups. Decomposition of states, Texts and Monographs in Physics, Springer-Verlag, Berlin, 1987.

[13] Deitmar A., van Dijk G., Trace class groups, J. Lie Theory 26 (2016), 269-291, arXiv:1501.02375.

[14] van Dijk G., Introduction to harmonic analysis and generalized Gelfand pairs, de Gruyter Studies in Mathematics, Vol. 36, Walter de Gruyter \& Co., Berlin, 2009. 
[15] van Dijk G., Neeb K.-H., Salmasian H., Zellner C., On the characterization of trace class representations and Schwartz operators, J. Lie Theory 26 (2016), 787-805, arXiv:1512.02451.

[16] Dobbins J.G., Well bounded semigroups in locally compact groups, Math. Z. 148 (1976), 155-167.

[17] Driver B.K., Heat kernels measures and infinite dimensional analysis, in Heat Kernels and Analysis on Manifolds, Graphs, and Metric Spaces (Paris, 2002), Contemp. Math., Vol. 338, Amer. Math. Soc., Providence, RI, 2003, 101-141.

[18] Fang S., Canonical Brownian motion on the diffeomorphism group of the circle, J. Funct. Anal. 196 (2002), 162-179.

[19] Fröhlich J., Osterwalder K., Seiler E., On virtual representations of symmetric spaces and their analytic continuation, Ann. of Math. 118 (1983), 461-489.

[20] Glimm J., Jaffe A., Quantum physics. A functional integral point of view, Springer-Verlag, New York Berlin, 1981.

[21] Gordina M., Riemannian geometry of $\operatorname{Diff}\left(S^{1}\right) / S^{1}$ revisited, in Stochastic Analysis in Mathematical Physics, World Sci. Publ., Hackensack, NJ, 2008, 19-29, math-ph/0510092.

[22] Gross L., Harmonic functions on loop groups, Astérisque 252 (1998), Exp. No. 846, 5, 271-286.

[23] Hida T., Brownian motion, Applications of Mathematics, Vol. 11, Springer-Verlag, New York - Berlin, 1980.

[24] Hilgert J., A note on Howe's oscillator semigroup, Ann. Inst. Fourier (Grenoble) 39 (1989), 663-688.

[25] Hilgert J., Neeb K.-H., Lie semigroups and their applications, Lecture Notes in Math., Vol. 1552, SpringerVerlag, Berlin, 1993.

[26] Hilgert J., Ólafsson G., Causal symmetric spaces. Geometry and harmonic analysis, Perspectives in Mathematics, Vol. 18, Academic Press, Inc., San Diego, CA, 1997.

[27] Hofmann K.H., Lie algebras with subalgebras of co-dimension one, Illinois J. Math. 9 (1965), 636-643.

[28] Hofmann K.H., Hyperplane subalgebras of real Lie algebras, Geom. Dedicata 36 (1990), 207-224.

[29] Holden H., Øksendal B., Ubøe J., Zhang T., Stochastic partial differential equations. A modeling, white noise functional approach, 2nd ed., Universitext, Springer, New York, 2010.

[30] Howe R., The oscillator semigroup, in The Mathematical Heritage of Hermann Weyl (Durham, NC, 1987), Proc. Sympos. Pure Math., Vol. 48, Amer. Math. Soc., Providence, RI, 1988, 61-132.

[31] Jaffe A., Ritter G., Quantum field theory on curved backgrounds. I. The Euclidean functional integral, Comm. Math. Phys. 270 (2007), 545-572, hep-th/0609003.

[32] Jaffe A., Ritter G., Quantum field theory on curved backgrounds. II. Spacetime symmetries, arXiv:0704.0052.

[33] Jorgensen P.E.T., Analytic continuation of local representations of Lie groups, Pacific J. Math. 125 (1986), 397-408.

[34] Jorgensen P.E.T., Analytic continuation of local representations of symmetric spaces, J. Funct. Anal. 70 (1987), 304-322.

[35] Jorgensen P.E.T., Ólafsson G., Unitary representations of Lie groups with reflection symmetry, J. Funct. Anal. 158 (1998), 26-88, funct-an/9707001.

[36] Jorgensen P.E.T., Ólafsson G., Unitary representations and Osterwalder-Schrader duality, in The Mathematical Legacy of Harish-Chandra (Baltimore, MD, 1998), Proc. Sympos. Pure Math., Vol. 68, Amer. Math. Soc., Providence, RI, 2000, 333-401, math.FA/9908031.

[37] Jørsboe O.G., Equivalence or singularity of Gaussian measures on function spaces, Various Publications Series, Vol. 4, Matematisk Institut, Aarhus Universitet, Aarhus, 1968.

[38] Kakutani S., On equivalence of infinite product measures, Ann. of Math. 49 (1948), 214-224.

[39] Kirillov A.A., Lectures on the orbit method, Graduate Studies in Mathematics, Vol. 64, Amer. Math. Soc., Providence, RI, 2004.

[40] Klein A., Gaussian OS-positive processes, Z. Wahrscheinlichkeitstheorie und Verw. Gebiete 40 (1977), 115124.

[41] Klein A., The semigroup characterization of Osterwalder-Schrader path spaces and the construction of Euclidean fields, J. Funct. Anal. 27 (1978), 277-291.

[42] Klein A., Landau L.J., Singular perturbations of positivity preserving semigroups via path space techniques, J. Funct. Anal. 20 (1975), 44-82. 
[43] Lawson J.D., Maximal subsemigroups of Lie groups that are total, Proc. Edinburgh Math. Soc. 30 (1987), 479-501.

[44] Lawson J.D., Polar and Ol'shanskii decompositions, J. Reine Angew. Math. 448 (1994), 191-219.

[45] Lüscher M., Mack G., Global conformal invariance in quantum field theory, Comm. Math. Phys. 41 (1975), 203-234.

[46] Merigon S., Neeb K.-H., Ólafsson G., Integrability of unitary representations on reproducing kernel spaces, Represent. Theory 19 (2015), 24-55, arXiv:1406.2681.

[47] Neeb K.-H., On a theorem of S. Banach, J. Lie Theory 7 (1997), 293-300.

[48] Neeb K.-H., Holomorphy and convexity in Lie theory, de Gruyter Expositions in Mathematics, Vol. 28, Walter de Gruyter \& Co., Berlin, 2000.

[49] Neeb K.-H., Ólafsson G., Reflection positivity and conformal symmetry, J. Funct. Anal. 266 (2014), 21742224, arXiv:1206.2039.

[50] Neeb K.-H., Ólafsson G., Reflection positive one-parameter groups and dilations, Complex Anal. Oper. Theory 9 (2015), 653-721, arXiv:1312.6161.

[51] Neeb K.-H., Ólafsson G., Reflection positivity for the circle group, J. Phys. Conf. Ser. 597 (2015), 012004, 16 pages, arXiv:1411.2439.

[52] Neeb K.-H., Ørsted B., Representation in $L^{2}$-spaces on infinite-dimensional symmetric cones, J. Funct. Anal. 190 (2002), 133-178.

[53] Nelson E., Analytic vectors, Ann. of Math. 70 (1959), 572-615.

[54] Nelson E., Feynman integrals and the Schrödinger equation, J. Math. Phys. 5 (1964), 332-343.

[55] Nelson E., The free Markoff field, J. Funct. Anal. 12 (1973), 211-227.

[56] Ólafsson G., Analytic continuation in representation theory and harmonic analysis, in Global Analysis and Harmonic Analysis (Marseille-Luminy, 1999), Sémin. Congr., Vol. 4, Soc. Math. France, Paris, 2000, 201233.

[57] Osterwalder K., Schrader R., Axioms for Euclidean Green's functions, Comm. Math. Phys. 31 (1973), 83-112.

[58] Osterwalder K., Schrader R., Axioms for Euclidean Green's functions. II, Comm. Math. Phys. 42 (1975), 281-305.

[59] Poguntke D., Well-bounded semigroups in connected groups, Semigroup Forum 15 (1977), $159-167$.

[60] Schrader R., Reflection positivity for the complementary series of SL(2n, C), Publ. Res. Inst. Math. Sci. 22 (1986), 119-141.

[61] Schwartz L., Sous-espaces hilbertiens d'espaces vectoriels topologiques et noyaux associés (noyaux reproduisants), J. Analyse Math. 13 (1964), 115-256.

[62] Schwartz L., Radon measures on arbitrary topological spaces and cylindrical measures, Tata Institute of Fundamental Research Studies in Mathematics, Vol. 6, Oxford University Press, London, 1973.

[63] Segal I.E., Ergodic subgroups of the orthogonal group on a real Hilbert space, Ann. of Math. 66 (1957), 297-303.

[64] Simon B., The $P(\varphi)_{2}$ Euclidean (quantum) field theory, Princeton Series in Physics, Princeton University Press, Princeton, N.J., 1974.

[65] Trèves F., Topological vector spaces, distributions and kernels, Academic Press, New York - London, 1967.

[66] Varadarajan V.S., Geometry of quantum theory, 2nd ed., Springer-Verlag, New York, 1985.

[67] Warner G., Harmonic analysis on semi-simple Lie groups. I, Die Grundlehren der mathematischen Wissenschaften, Vol. 188, Springer-Verlag, New York - Heidelberg, 1972.

[68] Yamasaki Y., Measures on infinite-dimensional spaces, Series in Pure Mathematics, Vol. 5, World Scientific Publishing Co., Singapore, 1985. 